\title{
SERIOUS GAMES TO STUDY THE INFLUENCE OF WILDLIFE DEVALUATION STRATEGIES ON HUNTER BEHAVIOUR
}

\author{
BY \\ SEAN ANTHONY RUDMAN
}

\begin{abstract}
A thesis
submitted to the Victoria University of Wellington

in fulfilment of the requirements for the degree of

Master of Science
\end{abstract}

Victoria University of Wellington

2019 
"Earth provides enough to satisfy every man's need but not for every man's greed"

\section{- Mahatma Gandhi}

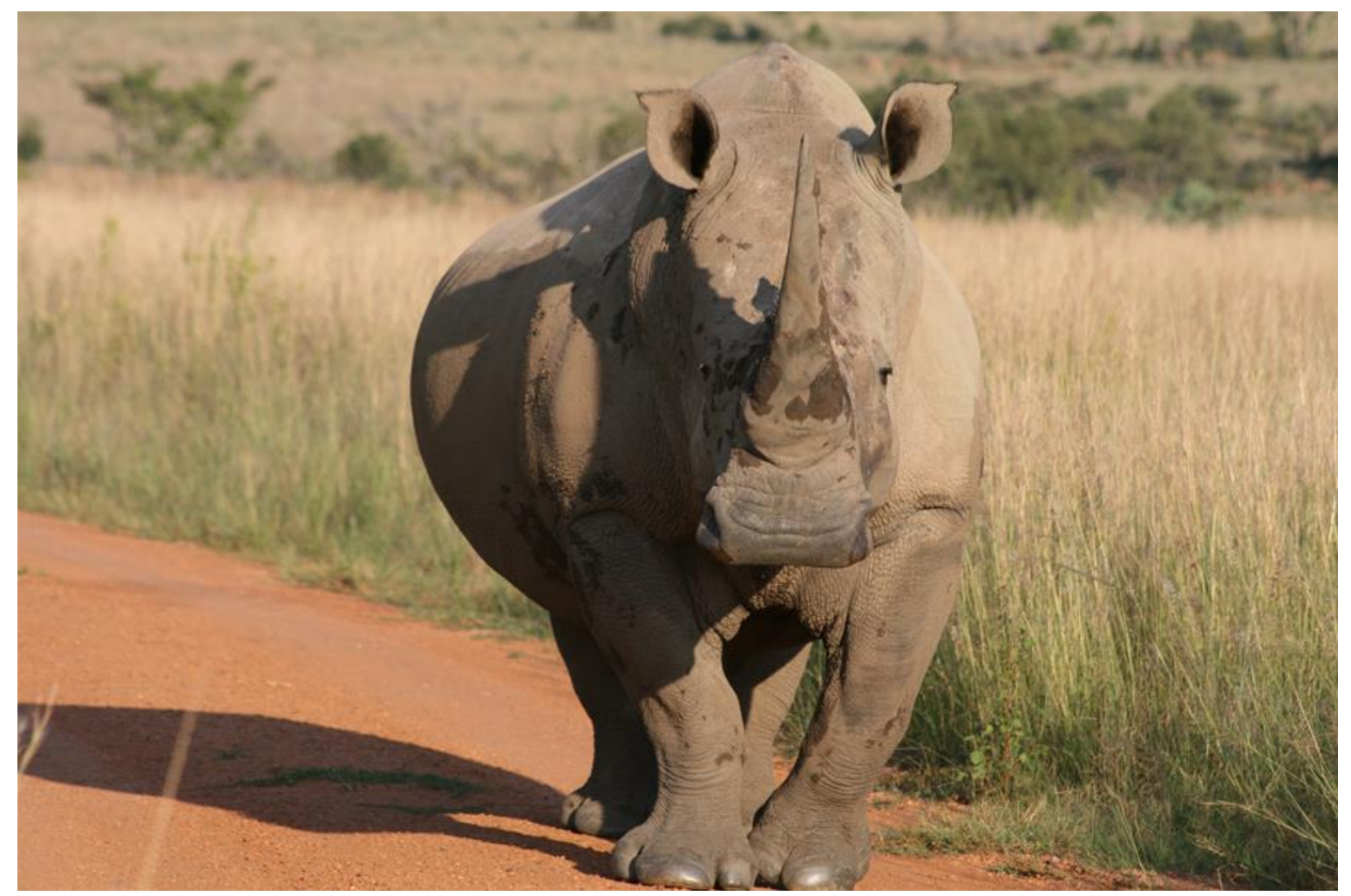

Image of a White rhino (Ceratotherium simum) captured by mum, Lynne Rudman, in South Africa. 
This thesis was conducted under the supervision of

Associate Professor Wayne Linklater (primary supervisor)

Centre for Biodiversity and Restoration Ecology

School of Biological Sciences

Victoria University of Wellington

Wellington, New Zealand

and

Associate Professor Jack Robles (secondary supervisor)

School of Economics and Finance

Victoria University of Wellington

Wellington, New Zealand 


\section{Abstract}

The international illegal wildlife trade (IWT) threatens countless species globally. Many solutions to the IWT have been proposed and implemented, the most common being increased security. However, security on its own has been ineffective at protecting all wildlife. Wildlife commodity devaluation strategies have also been proposed and trialled as a complement or substitute to security. The strategy has primarily been applied to protect rhinoceros populations by, for example, dehorning them. The apparently logical expectation is that reducing the value of rhinos' horns will discourage hunters and protect rhino. Either hunters will choose not to hunt a population with devalued animals or choose not to kill one when it is found. Unexpectedly, however, theory and anecdotal evidence suggest that devaluation might fail, and may even encourage hunting and the killing of devalued animals. This apparently illogical outcome is the subject of this thesis. Games and choice-based surveying were used to study the behaviour of people hunting a commodity for financial gain. These methods were used to understand why devaluation strategies might fail to protect wildlife and to understand when they might be beneficial.

Two games and a choice-based survey posing different hunting scenarios were developed to measure hunter behaviour and test hunters' responses to risk, value and devaluation. Lucky-dip games were rapid, highly replicated games used to test the impact of variation in devaluation and security strategies across multiple populations. Thirty-three lucky-dip games were conducted, each with ten members of the public participating. They were conducted at community events (e.g. fairs and galas) across Wellington Region in February and March of 2018. Scavenger-hunt games, to elicit more complex behaviours and interactions among participants, were conducted over a longer time and larger area but were, therefore, also less replicated. Four of these games were conducted with between 8-20 members of outdoor recreation clubs. They were conducted at public parks across Wellington Region throughout 2018. And lastly, an online scenario choice-based survey presented members of recreational hunting clubs with hypothetical scenarios where the value and likelihood of a successful hunt varied. The survey was distributed through hunting organisations and received 333 responses. The three research methods presented similar scenarios but used different formats to test my ideas among a diverse population of people. Each method involved participants hunting protected items that varied in value with some items having been devalued. Measuring for the trade-offs that people make between risk and 
reward when making hunting decisions, including choices about where to hunt, whether to kill and, if they did, whether to harvest a commodity, was of particular interest.

Devaluation failed to protect commodities and increase commodity survival. In both games, hunters chose to 'kill' devalued commodities. Of the devalued items located by hunters, $74 \%$ and 100\% were 'killed' in the lucky-dip and scavenger-hunt games, respectively. This appears to be because risk increased people's perceived value of partially devalued commodities. Low-risk lucky-dip games resulted in $44 \%$ more devalued items being kept by participants compared to high-risk games. Additionally, devaluation reduced people's perceptions of risk. Compared to lucky-dip games with just risk, games that included devaluation caused a 10\% drop in survival at the highest-risk treatment. Moreover, when devalued commodities were worthless in the lucky-dip games, $27 \%$ more were 'killed' compared to when devalued commodities were worth $25 \%$ of the full-value commodity. Therefore, contrary to expectations, partial devaluation may be more successful than complete devaluation. Greater rates of devaluation were also met with greater variation in commodity survival between games. Coefficients of variation for commodity survival increased from $12 \%$ to $41 \%$ as devaluation rates increased from zero to $100 \%$. Moreover, respondents to the survey ranked the most devalued population (90\%) highest $36 \%$ of the time, but also lowest $35 \%$ of the time. Thus, peoples' responses to devaluation vary. Risk was a more effective and consistent regulator of hunting behaviour. Average commodity survival was $88 \%$ at maximum security treatments but was only $65 \%$ at maximum devaluation treatments.

My experimental games and surveys do not support the expectation that reducing the value of rhinos' horns will discourage hunters and protect rhino. The IWT is a complex socio-economic system, and human behaviour is varied in response to risk and reward. Devaluation introduces a complex interaction between the two, rendering risk and devaluation less effective in some circumstances. Every population and situation is unique, and the effectiveness of devaluation will be context-specific. Finally, the research demonstrates that serious games can be applied to the study of criminal hunting behaviour. Other difficult-to-study human subjects and systems could benefit from greater use of similar methodologies. 


\section{Acknowledgements}

Firstly, I would like to thank my primary supervisor Associate Professor Wayne Linklater who has provided endless support and encouragement, not only during this thesis, but over the last three years. You have gone above and beyond the role of supervisor, encouraged me to push myself, and provided me with multiple opportunities within and outside the scope of my Masters. Your open-door policy was very much appreciated, as was your willingness to sit and discuss my research with me, sometimes for hours in unscheduled meetings. I would also like thank my secondary supervisor Associate Professor Jack Robles for assisting and supporting me with the economic component of the project.

The research would not have been possible without funding. Thank you to the Centre for Biodiversity and Restoration Ecology for funding the research, and for awarding me a grant to attend and present my research at the Society for Conservation Biology $5^{\text {th }}$ Oceania Congress. I would also like to acknowledge the importance of the Victoria University Masters by thesis Scholarship, the Victoria Graduate Award and the Alison Morton Scholarship in Ecology/Marine Biology which were awarded to me. These awards were vital in enabling me to support myself during my Masters.

I had many people assist me in conducting the games. Thank you to my partner Ella, brother Neil, flatmates Tessa, Kate and Anjela, and friends Jarrod, Ethan, Jake, Elle, Daniel and Alex for giving up your time to help me run the games. Your assistance was crucial, and I cannot thank you enough.

Thank you to the organisers of the public events where games were conducted and the organisations who agreed to distribute the survey or have me recruit participants for the games. The participants themselves also cannot go unmentioned. Without all of you, this research would not have been possible.

Thank you to my desk buddies Jacob, Justyna, Julie and Rachel for many laughs, and for always being there to support me and answer my questions. Other colleagues that deserve a mention for their friendship and making my Masters a more enjoyable experience include Mike Jackson, Alex Whyte, Roald Bomans, Jess Russell, Ben Harris, Tom Oosting, Bobby Lust, Chis Woolley, Regan Mackinlay, Johannes Fischer and Daniel Papworth.

A special thank you to Mike, Jarrod, Ella and my dad, Arthur, for agreeing to the unpleasant and laborious task of proofreading my thesis. Your fresh eyes and outside 
perspectives were extremely helpful. I would also like to express my appreciation to the School of Biological Sciences administration staff, particularly Mary Murray, Paul Marsden and Mark Stephen, for their support and assistance.

Finally, thank you to my extremely supportive parents (Lynne and Arthur), brother (Neil) and partner (Ella). You support, encouragement and confidence in me was endless and invaluable. To my parents, this thesis represents my last five years of university education which would not have been possible without your support - both financial and emotional. My achievements - past, present and future - are a credit to the fantastic, loving parents that you are. 


\section{Table of Contents}

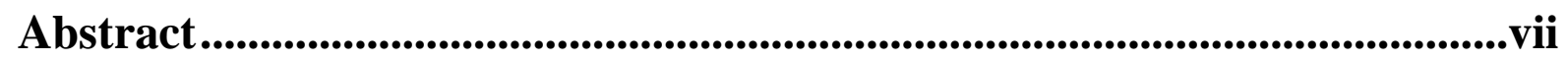

Acknowledgements..........................................................................................ix

Table of Contents ...................................................................................................xi

Table of Figures....................................................................................................................

Table of Tables ........................................................................................................... xvii

1 Introduction ...............................................................................................19

1.1 International trade in high-value wildlife commodities ........................................21

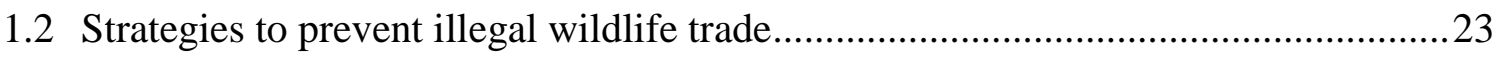

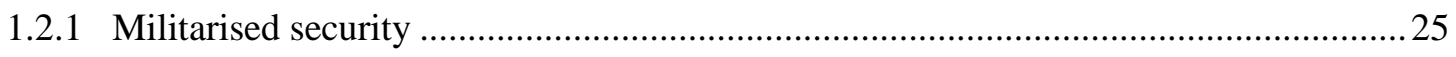

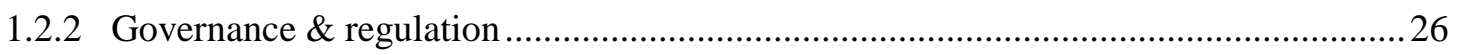

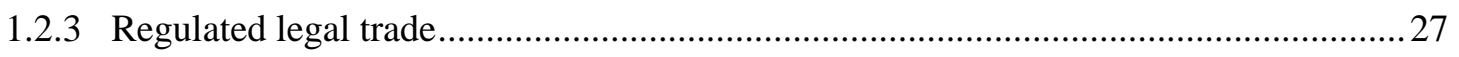

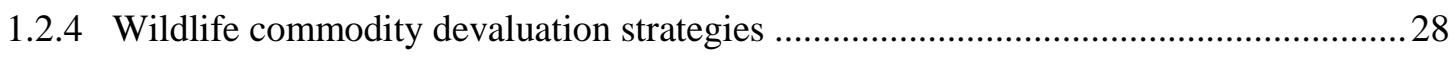

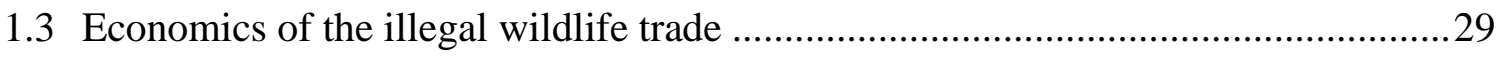

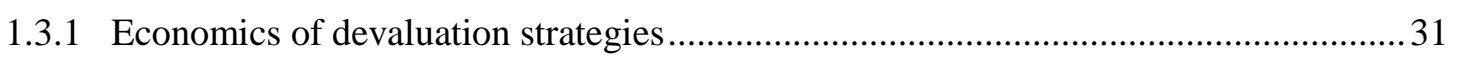

1.4 Behavioural modelling \& games as models .......................................................... 34

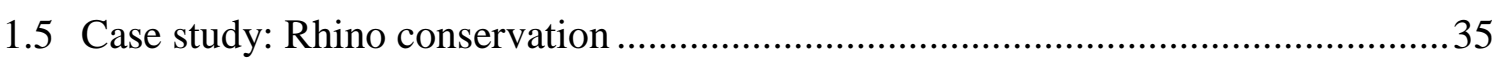

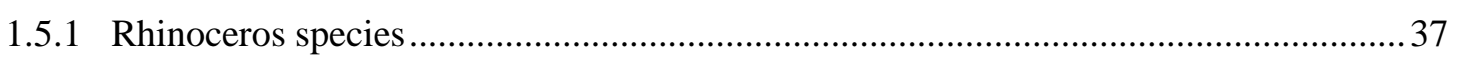

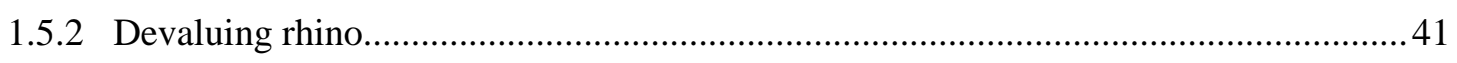

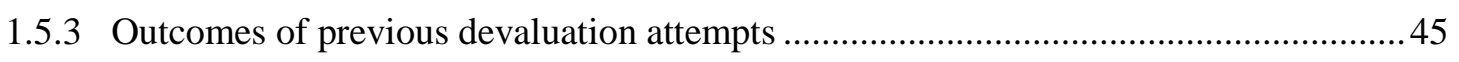

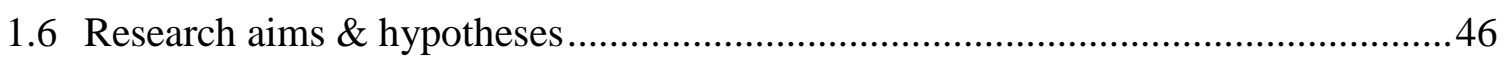

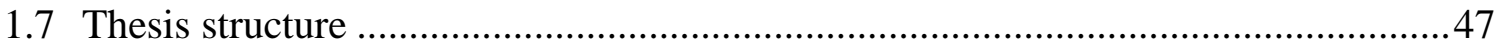

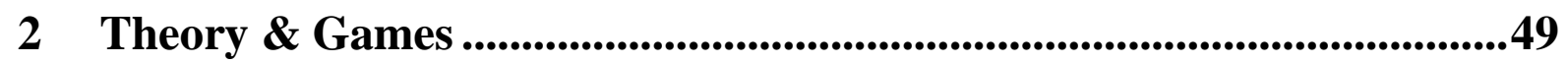

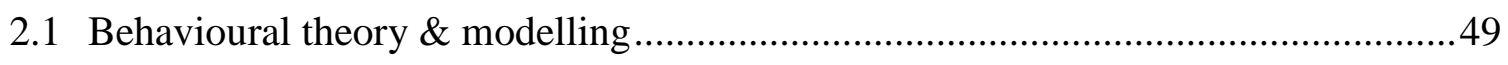

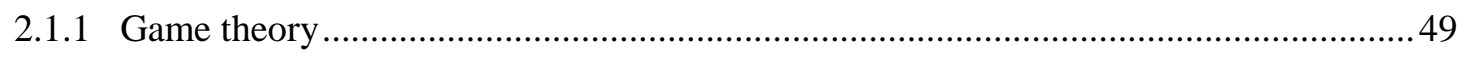

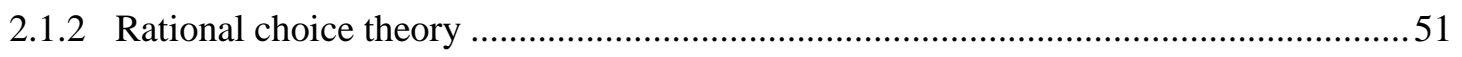

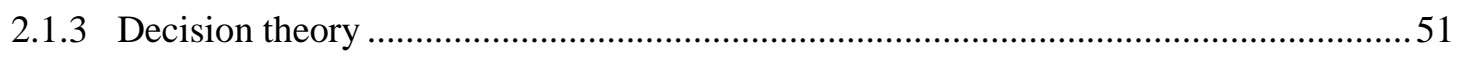

2.2 Modelling hunter behaviour under devaluation strategies ...................................52

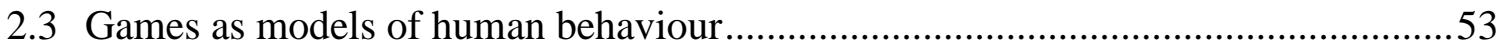

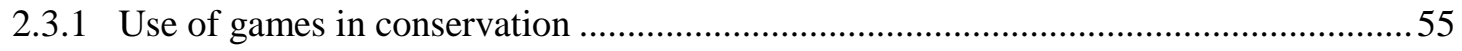




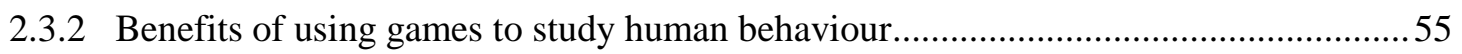

2.3.3 Difficulties of using games to study human behaviour.................................................57

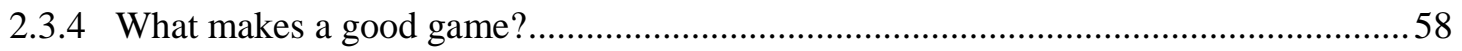

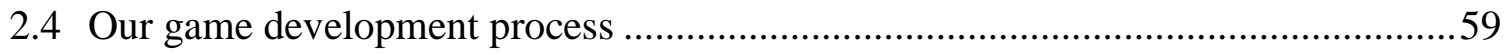

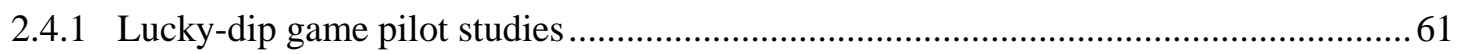

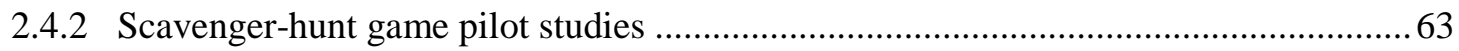

2.4.3 Improvements made during the development process .................................................65

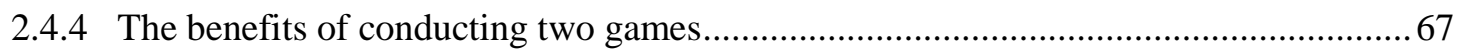

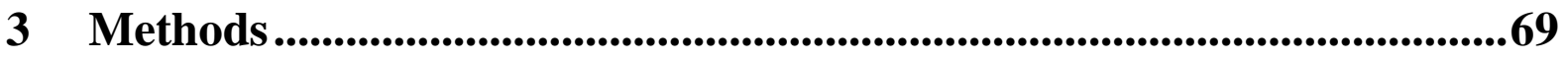

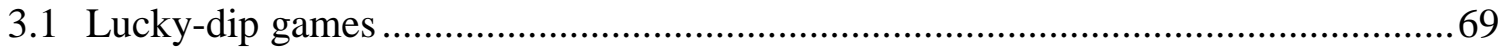

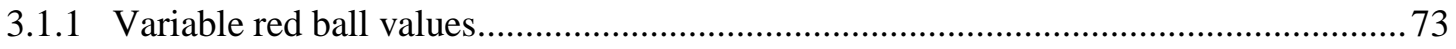

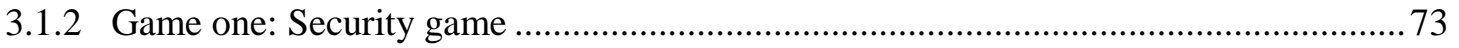

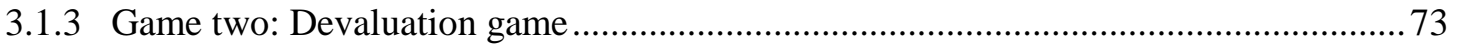

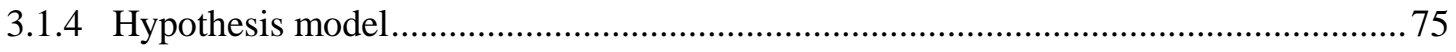

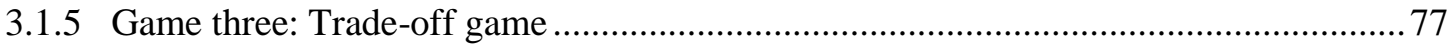

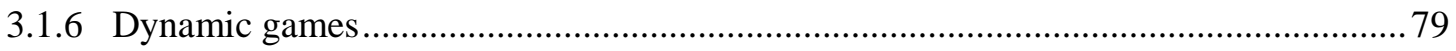

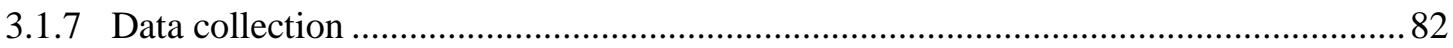

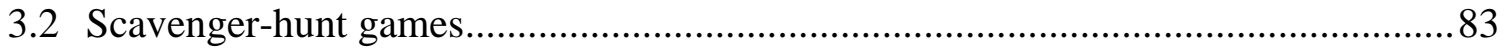

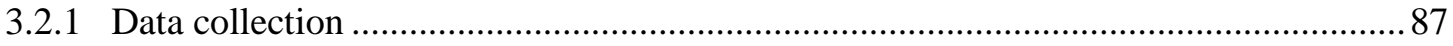

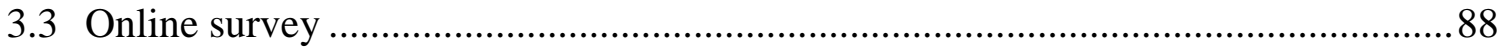

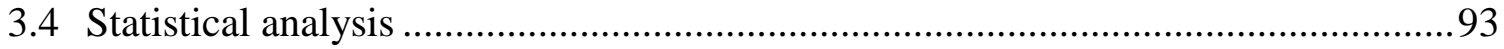

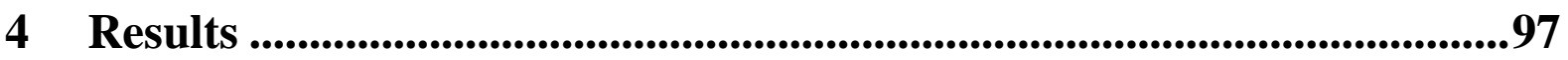

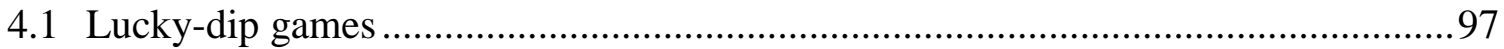

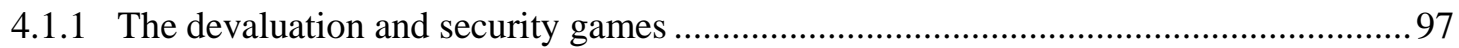

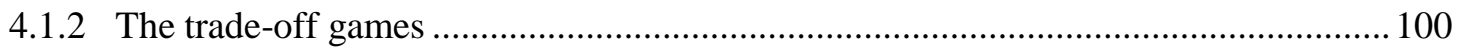

4.1.3 Total selections and blue ball selections ……........................................................ 103

4.1.4 Hunter treatment of devalued balls ......................................................................... 107

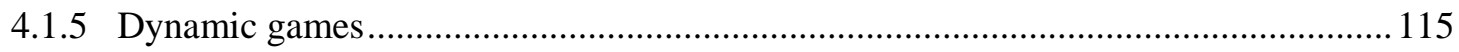

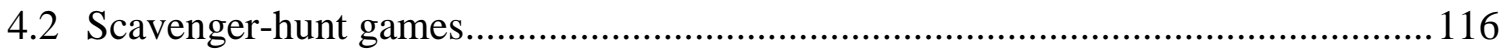

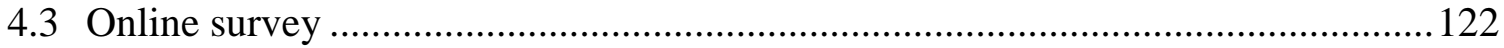

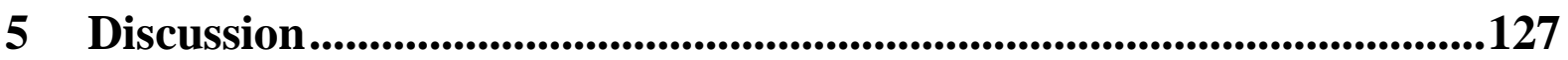

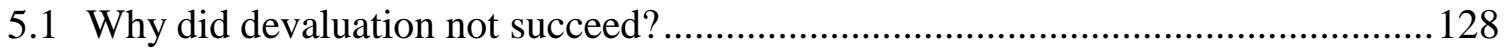

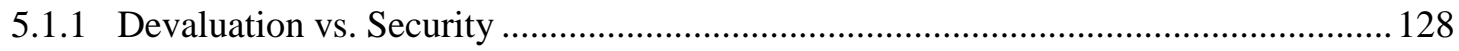


5.1.2 Risk aversion vs. Risk tolerance

5.1.3 Changes in perceptions of risk and value................................................................ 131

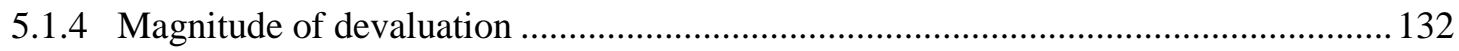

5.1.5 Devaluation did not demotivate participants............................................................ 133

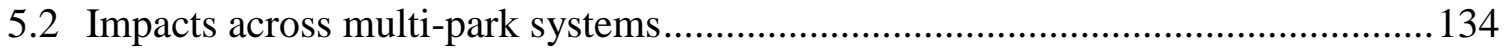

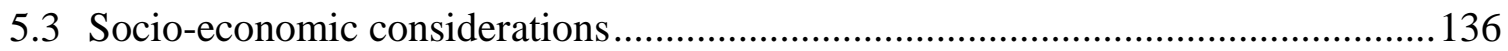

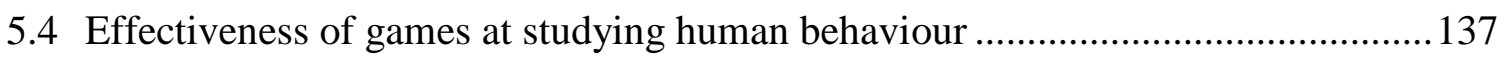

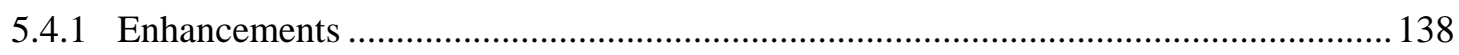

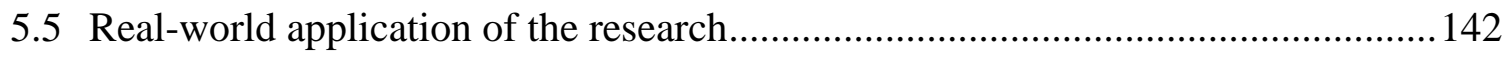

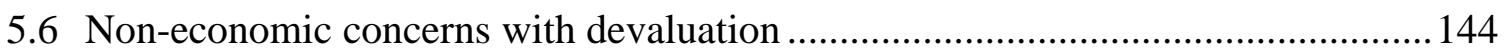

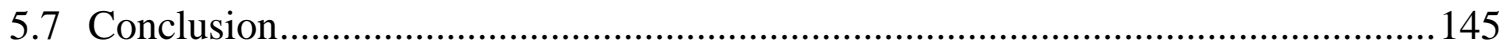

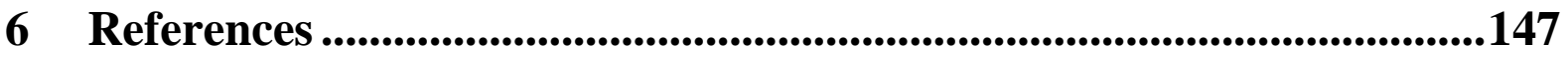

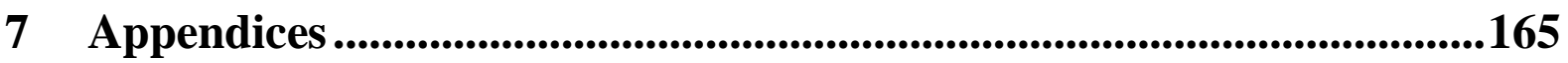





\section{Table of Figures}

Figure 1.1. A graphical example of inelastic demand. Recreated from Challender and MacMillan (2014).

Figure 1.2. The escalation of illegal rhino hunting in South Africa between 2000 and 2016 (Biggs et al., 2013; Emslie et al., 2016; Trump, 2017).

Figure 1.3. Trends in rhinoceros numbers for (a) African species and (b) Asian species (Emslie, 2004; Emslie \& Brooks, 1999; Emslie et al., 2007; Emslie, Milliken, \& Talukdar, 2013; Emslie et al., 2016; Foose \& van Strien, 1997; Milliken, Emslie, \& Talukdar, 2009)..40

Figure 1.4. The dehorning process (Wildlife ACT, 2018) 42

Figure 1.5. The horn infusion process (Rhino Rescue Project, n.d.-b)................................ 44

Figure 2.1. Results of the first lucky-dip pilot study (Linklater \& Rudman, 2017).

Figure 2.2. Results of the first scavenger-hunt pilot study (Linklater, Rudman, \& Jackson, 2017).

Figure 2.3. Results of the second scavenger-hunt pilot study (Linklater et al., 2017).... 64

Figure 3.1. A diagram of how the stall was set up at the public events. .70

Figure 3.2. Decision tree showing a participant's possible actions upon selecting a ball depending on which coloured ball is selected, and the outcomes of each action.

Figure 3.3. An example of what the hypothesis model for predicting commodity survival may look like.

Figure 3.4. Maps of the three sites where scavenger-hunt games were conducted. Maps retrieved from Google Maps (Imagery: Google 2018, Map Data: Google 2018).

Figure 3.5. The four parks that were presented to respondents in Section 1 of the survey. Images retrieved from Honeycutt and Orndorff (2016).

Figure 3.6. The four parks that were presented to respondents in Section 2 of the survey. Images retrieved from Honeycutt and Orndorff (2016).

Figure 4.1. The average survival of balls (out of 10) in the devaluation (red) and security (blue) lucky-dip games. 
Figure 4.2. The expected average survival for each box based on the hypothesis model versus the observed average survival for each box in the trade-off games

Figure 4.3. The average number of balls selected, and the average number of blue balls selected at each box in trade-off games.

Figure 4.4. The number of blue balls placed in each box of the trade-off games and the proportion of blue balls starting in each box of the trade-off game

Figure 4.5. The percentage of red balls kept, returned and discarded for each of the red ball values. 108

Figure 4.6. The percentage of red balls kept, returned and discarded in devaluation and tradeoff games

Figure 4.7. The percentage of red balls kept, returned and discarded for each of the five sites.

Figure 4.8. New Zealand census data of the percentage of residents in each suburb where games were conducted (profile.idnz.co.nz, 2018a; profile.idnz.co.nz., 2018b)

Figure 4.9. The average number of stakes 'killed' (delivered or laid down) in the scavengergames of each devaluation level.

Figure 4.10. The average Likert scale score of participant motivation for each devaluation level

Figure 4.11. The average number of steps per participant pair at each devaluation level....120

Figure 4.12. The average number of steps per participant pair during each game stage, with all devaluation levels combined.

Figure 4.13. The number of times each park was selected in each of the six pairwise comparisons.

Figure 4.14. The percentage of each rank score $(1=$ most preferred; $4=$ least preferred $)$ received by each of the four parks.

Figure 4.15. The percentage of respondents who selected return and discard when there were more yearlings in the presented populations, and more stags in the presented populations. The red dashed line marks the $50 \%$ threshold. 


\section{Table of Tables}

Table 2.1. The number and distribution of yellow, red and blue balls in each of the five boxes in the lucky-dip pilot study games.

Table 3.1. The number and distribution of yellow and blue balls at the start of each (a) security game, and (b) devaluation game.

Table 3.2. The number and distribution of yellow, red and blue balls at the start of each lucky-dip trade-off game .78

Table 3.3. A summary of the number of games of each variation completed. .81

Table 3.4. A summary of the type of organisation each game was conducted with, where each game was conducted, the number of participants and the devaluation percentage.

Table 3.5. Assuming the respondent selected a worthless yearling with their first selection, this table shows the chance that the participant would receive a stag on their next selection if they returned or discarded the yearling.

Table 4.1. Mann-Whitney post-hoc analysis $p$-values for the pairwise comparisons of total survival at each pair of boxes in (A) devaluation games, and (B) security games.

Table 4.2. Mann-Whitney post-hoc analysis $p$-values for the pairwise comparisons of total survival at each pair of boxes in trade-off games.

Table 4.3. The average survival of balls, the standard deviation, and the coefficient of variation $(\mathrm{CV})$ for survival at each box in the trade-off games. 102

Table 4.4. Mann-Whitney post-hoc analysis $p$-values for the pairwise comparisons for each pair of boxes of (A) total selections in trade-off games, and (B) number of blue ball selections in trade-off games.

Table 4.5. The results of the post-hoc tests for the chi-square test of red ball outcome and red ball value. The values presented are residuals with a Bonferroni correction. 108

Table 4.6. The results of the post-hoc tests for the chi-square test of red ball outcome and game type. The values presented are residuals with a Bonferroni correction. 110 
Table 4.7. The results of the post-hoc tests for the chi-square test of red ball outcome and site. The sites are ordered from least deprived (left) to most deprived (right). The values presented are residuals with a Bonferroni correction.

Table 4.8. Post-hoc Tukey test results for the ANOVA of the average number of steps per participant pair across the three game stages.

Table 4.9. The Bradley-Terry model output $p$-values for each park pairwise comparison... 122

Table 4.10. The results of the post-hoc tests for the chi-square test of park and ranking. The values presented are residuals with a Bonferroni correction. 


\section{Introduction}

Hunting for the illegal wildlife trade (IWT) became a serious global issue during the $20^{\text {th }}$ century and has continued to grow into the $21^{\text {st }}$ century (Ayling, 2013; Cooney et al., 2017). The trade has an estimated annual value of between USD \$5 billion and USD \$20 billion, potentially making it the second largest illegal market after drug trafficking (Rosen \& Smith, 2010; Warchol, 2004). An estimated 33\% of mammals and birds, and $75 \%$ of fisheries are threatened by the IWT (Rivalan et al., 2007). This threat is not limited to fauna. An estimated 1,000 species of timber are threatened with extinction due to the IWT (Rivalan et al., 2007).

The international regulation and criminalisation of hunting and trade in wildlife arose through the formation of the United Nations Convention on International Trade in Endangered Species of Wild Fauna and Flora (CITES) in 1973, the main regulatory body of the IWT (Ayling, 2013). Many international organisations and NGOs have subsequently become involved in attempting to regulate the IWT. Along with CITES, the World Wildlife Fund (WWF), TRAFFIC and the International Union for Conservation of Nature (IUCN) are a few of the organisations attempting to regulate the IWT.

IWT is driven by complex social, cultural and economic issues (Challender \& MacMillan, 2014). These issues include, but are not limited to, corruption (Bennett, 2015), poverty (Challender \& MacMillan, 2014) and cultural beliefs (Warchol, 2004). For example, up to $90 \%$ of consumed animal protein in west and central Africa comes from wild animals, and in Gabon, up to $72 \%$ of household income is acquired through hunting (Kurpiers et al., 2016). The rapid growth of IWT is often attributed to increased poverty in regions where wildlife commodities are harvested (source regions) such as Africa, coupled with increased affluence in regions where these commodities are retailed (consumer regions) such as southeast Asia (Challender \& MacMillan, 2014). Increasing poverty in source regions drives people to seek out supplementary or replacement income sources, including through illegal means, especially when income opportunities are scarce (Challender \& MacMillan, 2014). The simultaneous increasing affluence in consumer regions means that more people are able to afford and acquire these commodities, thus motivating and resourcing the illegal market (Challender \& MacMillan, 2014).

Many different illegal wildlife markets exist. These range from local markets, such as those for bushmeat, that target a wide range of species, to high-value commodity markets that target fewer species intensively (Duffy et al., 2016; Kurpiers et al., 2016). Local, low-value 
wildlife markets, such as the bushmeat trades of Africa and Asia, are primarily driven by subsistence and poverty. The main drivers of the bushmeat trade in Africa are protein and income requirements (Brashares et al., 2004). In west-Africa the primary source of protein is fish, with agriculture and fishing important income sources (Brashares et al., 2004). The bushmeat trade increases in years of poor fishing and low agricultural yield as income and access to protein declines (Brashares et al., 2004). Households compensate by harvesting bushmeat (Brashares et al., 2004). Proposed solutions include stabilising the agricultural industries and regulating the harvests of commercial and illegal fishing vessels of the coast of Africa (Brashares et al., 2004). This would ensure locals have a consistent income and access to fish, thereby reducing their need for bushmeat (Brashares et al., 2004). Solutions, like these, are made possible by understanding the complexity of the ecology and behaviour of the human populations involved.

Markets dealing in high-value wildlife commodities are particularly threatening to the species targeted. Examples of such species include elephant (Loxodanta africana) for ivory, pangolin (Manis spp.) for their scales and rhinoceros (Rhinocerotidae spp.) for their horns (Challender \& MacMillan, 2014). In the decade preceding 2014, over one million pangolins were illegally traded, more than any other protected wild animal (Challender, Waterman, \& Baillie, 2014). Between 2010 and 2012 an estimated 100,000 elephants were killed for their ivory (Wittemyer et al., 2014), and between 2007 and 2014 the illegal hunting of rhino increased over 9,000\% (Annecke \& Masubelele, 2016). Identifying solutions to high-value IWT may require research similar to that applied to low-value bushmeat hunting to understand the motivations and behaviour of hunters.

\section{Text Box 1.1. Definitions of hunting and harvest}

People who illegally hunt wildlife are commonly referred to as "poachers". However, due to the negative connotations and historiographic context of the term "poach", I have used the phrase "illegal hunting" instead. The term "poach" is associated with the connotation of the impoverished taking from the privileged. During the 1000's, hunting became a popular pastime of English royalty (Manning, 1993). As wildlife numbers declined through hunting, royalty began to protect wildlife by forming royal forests, hunting regulations and punishments (Griffin, 2007). However, social inequality and poverty forced people to engage in criminal behaviours in the pursuit of food (Hay, 1975). The regulation of hunting was continued by the first Parliament of England with the first wildlife law being enacted 
by parliament in 1389 (Manning, 1993). Laws that followed included the Game Act of 1831 that distinguished poaching deer from other poaching, and the infamous Black Act of 1723 that reintroduced the death penalty for poaching deer (Griffin, 2007; Manning, 1993). These laws were defended as measures "to prevent persons of inferior rank, from squandering that time, which their station in life requireth to be more profitably employed" (Hay, 1975, p. 191). This statement demonstrates the aristocratic culture associated with hunting, and the discriminatory origins of illegal hunting and the term "poaching".

The term "hunt" is used rather than the term "harvest". While many illegally traded species are harvested alive, many well-known examples involve species that are killed. Griffin (2007, p. 5) defines hunting as "the practice of chasing wild animals for the purpose of profit or sport". While this definition encompasses killing the animal, it also encompasses non-lethal hunting. The term "harvest" is occasionally used when referring to the general collection of wildlife, or if an example of non-lethal IWT is discussed. The term "trafficking" is used as per its definition of trading in something illegal.

\subsection{International trade in high-value wildlife commodities}

The greater threat posed to species of high-value by illegal trade is because, first, they provide greater financial incentives to illegal hunters. Second, they are more likely to be traded internationally and are thus more difficult to regulate (Schneider, 2008). Lastly, their high-value and international trade leads to associations with other organised crime networks such as drug trafficking and terrorism (Crosta \& Sutherland, 2016). These factors mean that high-value wildlife commodity markets are likely to result in global, not just local, extinctions of species.

In some cases, the potential rewards of trading in wildlife are so high that even the risk of death is insufficient to discourage hunting. In some countries, such as Zimbabwe, illegal hunting continues despite the adoption of "shoot-to-kill" policies for suspected illegal hunters (Duffy, 2014). Violence and conflict go hand-in-hand with this trade because hunters are highly motivated to obtain high-value commodities through any means necessary, including the use of violence (Büscher \& Ramutsindela, 2016). Additionally, when a market is created around a valuable illegal commodity, the initial unorganised criminals are often replaced by organised criminal syndicates. This was true for the drug trade, and is now true for the IWT (Milliken et al., 2012). The formation of criminal syndicates creates increased 
organisation and efficiency of the criminal activity, together with increased funding, corruption and violence (Dudley, Stolton, \& Elliott, 2013). The trade and criminals become increasingly weaponised as revenue to purchase weapons increases and the syndicates form associations with other criminal organisations and markets (Dudley et al., 2013).

Many uses for wildlife commodities are utilitarian - for practical uses such as consumption or medicine. However, some uses are hedonic - for non-practical uses such as symbolic, visual or emotional appeal (Dang Vu \& Nielsen, 2018). Hedonic values have driven some high-value wildlife commodities to become symbols of societal status (Ayling, 2013). While rhino horn has utilitarian values through its use in traditional Chinese medicine (TCM), it is also associated with hedonic values (Ayling, 2013). In Yemen, rhino horn is carved in to dagger handles that Yemeni men carry to symbolise their societal status (Ayling, 2013). Rhino horn has also become a societal status symbol in other cultures, especially recently in east Asia (Dang Vu \& Nielsen, 2018). This demand is primarily driven by the high-value of the commodity (Dang Vu \& Nielsen, 2018). Rhino horn is purchased and displayed to communicate a person's wealth and status (Dang Vu \& Nielsen, 2018). Rhino horn is also perceived as an expensive and elite gift used to gain support from those in power (Dang Vu \& Nielsen, 2018). In Vietnam, rhino horn mixed with wine is referred to as the drink of millionaires (Ayling, 2013).

Another factor that makes the IWT a serious concern is its international nature. Large international markets tend to involve many participants (Nijman, 2010). This means that there can be many traders, sellers and buyers involved in the trade of a single commodity (Nijman, 2010). This creates to redundancy in the system ensuring that any participants lost through enforcement are quickly replaced (Williams, 2001). Creating redundancy in networks is a common phenomenon in the drug trade (Kenney, 2007). Additionally, commodities may be conveyed along multiple, complex routes that are geographically widely distributed (Schneider, 2008). While the final destination for many wildlife products is Asia, many of these commodities will pass through intermediate destinations (Schneider, 2008). This allows for commodities to be amalgamated into single transport modes, for transport modes to be switched and for the commodity to be processed (e.g. the carving of ivory) (Schneider, 2008). All of these measures ensure that detecting and regulating the trade is difficult for enforcement agencies (Schneider, 2008). 
When illegal markets expand internationally, regulating the industry becomes very difficult. International organisations such as CITES regulate the international IWT, but have no authority to regulate domestic markets (Lemieux \& Clarke, 2009). Therefore, for the IWT to be regulated successfully, international collaboration between domestic law enforcement agencies is required. The difficulty is exacerbated when the different countries involved have different or even conflicting laws. For example, trading ivory is illegal within South Africa and internationally (Warchol, 2004). However, a large proportion of illegal ivory is trafficked to Japan, where domestic ivory trade remains legal (Bennett, 2015). This makes it difficult for the authorities of these countries to collaborate, for example through the sharing of intelligence (Haas \& Ferreira, 2015). Additionally, illegal hunting more commonly occurs in underdeveloped countries with corruption and poor governance (Challender \& MacMillan, 2014). Therefore, law enforcement co-operation between countries is difficult to achieve.

Finally, the IWT trade is directly linked to other illegal industries and markets, such as international drug trafficking (Schneider, 2008). The connections between these two markets include parallel trafficking routes, drugs being concealed within wildlife commodity consignments, and wildlife commodities and drugs being traded for each other (Schneider, 2008). There have also been reports that the IWT has been used to fund terrorist organisations (Duffy et al., 2016). In 2013, the Elephant Action League released the article stating that the terrorist group al-Shabaab was funded by the illegal ivory trade (Kalron \& Crosta, 2013). Al-Shabaab is a terrorist organisation based in east-Africa (predominantly Somalia) with ties to al-Qaeda (Crosta \& Sutherland, 2016). The original article was criticised for its lack of evidence. Subsequently, the Elephant Action League released a report stating the evidence used to draw these conclusions (Crosta \& Sutherland, 2016). The report clarifies that al-Shabaab also has other, larger funding sources and that it did not drive the illegal hunting of elephants (Crosta \& Sutherland, 2016). Nevertheless, it claims that alShabaab significantly benefited from this trade, and suggests a link between the IWT and terrorism (Crosta \& Sutherland, 2016).

\subsection{Strategies to prevent illegal wildlife trade}

Regulating the illegal hunting of high-value wildlife is difficult. This is due to these commodities having high values, the commodities being traded internationally and links to other illegal markets. Manipulating the economic benefits and risks of hunting illegally is difficult because the benefits have to be reduced substantially, or the risks have to be 
sufficiently large to outweigh the benefits (Cooney et al., 2017). All strategies to prevent illegal hunting either attempt to increase the risk to illegal hunters, or reduce the rewards, as shown in the risk-reward equation below (Equation 1.1; du Toit \& Anderson, 2013). Strategies to prevent illegal hunting aim to reduce illegal hunting pressure. Reducing the benefits received by the hunter achieves this, as does increasing the risk of death or arrest or the effort required to hunt.

Equation 1.1. Risk-reward equation of illegal hunting. The response variable is the level of illegal hunting pressure. The numerator is the benefits received by the illegal hunter. The denominator is comprised of two functions - the risk to the illegal hunter of being arrested or killed, and the effort required to hunt illegally (du Toit \& Anderson, 2013).

Illegal hunting pressure $=\frac{\text { Benefits received by hunter }}{\text { Risk of arrest or death } \times \text { Effort required to hunt }}$

Many solutions to preventing IWT have been proposed. Large scale initiatives such as education, poverty alleviation and community-based conservation strategies are widely accepted to be ultimate solutions to reducing IWT (Challender \& MacMillan, 2014; Challender, Wu, et al., 2014; Phelps, Biggs, \& Webb, 2016). Unfortunately, these large-scale initiatives are long-term strategies (Challender \& MacMillan, 2014). It could take generations for these strategies to substantially reduce the IWT, especially on a large scale (Challender \& MacMillan, 2014). Therefore, shorter-term solutions that can be implemented quickly and that rapidly reduce hunting and trade are often proposed to temporarily stem the huntinginduced decline of a species. Many short-term strategies that are used to prevent illegal hunting attempt to reduce animal deaths by intervening in the trade of illegal wildlife commodities. Some try to manipulate the market directly (e.g. selling contraband to depress commodity value) and some regulate harvesting (e.g., security to discourage illegal hunting) which can indirectly manipulate the market and trade (Haas \& Ferreira, 2015). 


\subsubsection{Militarised security}

Militarised law enforcement or security is the most common strategy used to reduce illegal hunting (Challender \& MacMillan, 2014). While law enforcement is vital in regulating IWT, alone it has not prevented illegal hunting and trade (Challender \& MacMillan, 2014; Pires \& Moreto, 2011). Some high-value species, such as elephant, already receive high levels of protection, yet they continue to be hunted and traded illegally (Challender \& MacMillan, 2014). In large and remote protected areas, effective enforcement is difficult due to the infrastructure and number of personnel required (Rosen \& Smith, 2010). Additionally, increasing hunting disincentives, such as security, in one area may increase the intensity of hunting in surrounding areas (Lindsey \& Taylor, 2011).

Militarised security is also highly criticised. It aims to increase the risk of hunting illegally and therefore demotivate illegal hunters (Lunstrum, 2014). This usually involves rangers and security personnel utilising military style weapons and tactics (Shaw \& Rademeyer, 2016). The growth of this strategy has given rise to the terms "green violence" and "green militarization" (Büscher \& Ramutsindela, 2016). In response, illegal hunters have adopted similar weapons and tactics (Cooney et al., 2017). This outcome has been compared to the "war on drugs" where increased enforcement has failed to regulate the trade and use of drugs (Challender \& MacMillan, 2014). The result is increasing violence and militarization to the point where the conflict between rangers and illegal hunters is now described as a 'war' (Lunstrum, 2014; Shaw \& Rademeyer, 2016). Many countries such as India and Botswana have adopted shoot-to-kill policies, which authorise security personnel to shoot suspected illegal hunters rather than detain them (Humphreys \& Smith, 2018). In some developing countries where illegal hunting is common, security is also hindered by poor governance and corruption of government officials and security personnel (Bennett, 2015; Challender \& MacMillan, 2014; Rosen \& Smith, 2010).

Continually increasing the intensity of militarised security is insufficient (Challender \& MacMillan, 2014). This strategy reduces the complex socio-economic character of the IWT into a simple law enforcement issue which does not deal to the fundamental drivers of the trade, such as culture, poverty and corruption (Challender \& MacMillan, 2014). Militarised security can also alienate local communities and oppose cultures who believe it is their right to harvest wildlife (Duffy et al., 2016). This can create disincentives for local people to engage in conservation (Challender \& MacMillan, 2014). 


\subsubsection{Governance and regulation}

Another strategy for reducing the IWT is through regulation and governance. As the IWT has expanded across international borders, international regulatory agencies have been created. CITES and TRAFFIC are the key organisations tasked with monitoring and regulating the IWT. CITES is an international treaty that provides a legal basis for governing wildlife trafficking (Rivalan et al., 2007). In 1973, a meeting of 80 countries resulted in the creation of CITES which has since grown to 176 signatory countries (Ayling, 2013). These countries are obligated to monitor the IWT and exert efforts to protect threatened species (Rosen \& Smith, 2010). To date CITES has classified over 33,000 species to three different threat levels (Angelici, 2016; Rivalan et al., 2007). Appendix I species receive complete trade bans except for in extreme circumstances (Rosen \& Smith, 2010). Examples include the 1977 trade ban for rhino horn and the 1989 trade ban for ivory (Bennett, 2015; Biggs et al., 2013). Appendix II species require a CITES permit for trade to occur, and appendix III species are those where one signing country has requested the assistance of other member countries to regulate its trade (Rosen \& Smith, 2010). CITES has issued complete trade bans to over 800 species (Pires \& Moreto, 2011).

TRAFFIC, the wildlife trade monitoring network, is a joint network between the IUCN and WWF (Rosen \& Smith, 2010). Formed in 1976, TRAFFIC works closely with CITES, to monitor wildlife trade and ensure that it does not become a conservation threat (Rosen \& Smith, 2010). It achieves this by studying and investigating IWT, and suggesting plans of action to protect globally threatened species (Rosen \& Smith, 2010).

Because CITES and TRAFFIC are international organisations they only have authority to regulate the international trade of wildlife products (Lemieux \& Clarke, 2009). While they can advise countries with regards to national policy and regulations, they have no jurisdiction to regulate domestic markets (Lemieux \& Clarke, 2009; Schneider, 2008). This is why many countries such as Japan, China and the United States can retain legal domestic markets for ivory despite the 1989 CITES trade ban (Bennett, 2015; Harvey, 2016). It is also why, in 2017, South Africa was able to legalise the domestic trade of rhino horn (Rubino, Pienaar, \& Soto, 2018). 


\subsubsection{Regulated legal trade}

Rather than imposing trade bans, regulating a legal trade for wildlife commodities is another proposed solution to reduce illegal hunting. It is suggested that legalised trade will increase the supply of the commodity by removing supply restrictions (Harvey, 2016). Increasing supply could occur through flooding the market with stockpiled commodities (Harvey, 2016). When a commodity becomes more accessible through increased supply, the market value of the commodity can decline (Biggs et al., 2013). The reduced value of the commodity would discourage illegal hunting because the relative risk of hunting would increase when the hunters' potential rewards decrease (Biggs et al., 2013). However, this would only be a temporary strategy because once stockpiles are exhausted the market can no longer be flooded. To overcome this, it is also suggested that regulated legal trade could provide an opportunity for illegally hunted species' to be farmed, particularly if the valuable commodity replenishes and can be harvested without harming the animal. This could allow for stockpiles to be continually replenished. For example, rhino can be dehorned without harming the animal and the horns regrow (Milner-Gulland, Beddington, \& Leader-Williams, 1992). This means that rhino can be farmed and have their horns episodically harvested (Dang Vu \& Nielsen, 2018). Farming could assist conservation by meeting consumer demand and reducing the hunting pressure on wild populations (Bennett, 2015). Additionally, the revenue raised through regulated trade could be reinvested into conservation (Bennett, 2015). In the case of rhino horn, CITES remains resolute on its intentional trade ban, but its inability to control domestic policies means countries can oppose them (Lemieux \& Clarke, 2009). In 2017 South Africa controversially reversed its domestic ban on rhino horn trade. This allowed John Hume, the world's largest private owner of rhino, to controversially conduct an online rhino horn auction (Murcott, 2017). Mr Hume is reported to own around 1,300 rhino that are regularly dehorned (Murcott, 2017). He is estimated to have stockpiled approximately 6 tonnes of rhino horn with an estimated value of US\$235 million (Murcott, 2017). He hopes to sell this stockpile and invest the revenue into protecting and growing his population (Murcott, 2017).

Unfortunately, previous attempts to legalise illegal wildlife markets have shown that the outcome is unpredictable. Abalone is an example of a failed legalisation attempt. The trade in abalone is a valuable industry that involves illegal collecting and trade of a threatened stock (Crookes, 2016). The abalone conservation framework allows law enforcement to confiscate illegally harvested abalone and sell it on the legal market to 
generate revenue for abalone conservation (Crookes, 2016). This is very similar to the framework proposed for a legal rhino horn market (Crookes, 2016). However, this framework resulted in law enforcement focussing on confiscation efforts to maximise revenue, rather than efforts to prevent the illegal collection of abalone (Crookes, 2016). Therefore, this framework is unlikely to result in the long-term conservation of abalone (Crookes, 2016). In addition, legal markets can have the opposite effect of increasing demand by expanding the legitimate market and increasing supply (Harvey, 2016). If regulated legal trade fails to reduce price, demand for the commodity could increase resulting in further hunting of protected and wild populations (Harvey, 2016). The one-off sales of ivory in 1998 and 2008 increased demand significantly, increasing the market value of ivory and causing an increase in the illegal hunting of elephants (Harvey, 2016).

\subsubsection{Wildlife commodity devaluation strategies}

Wildlife commodity devaluation strategies are proposed as a new and novel solution to reduce illegal hunting. While these strategies have been utilised for decades, they have recently received increased attention due to the development of new devaluation methods. Devaluation strategies aim to introduce hunting disincentives that are higher, or at least equal, to other hunting prevention strategies (Ferreira, Hofmeyr, et al., 2014). Devaluation creates a hunting disincentive by introducing the risk of locating a devalued animal and increasing the effort and cost required to locate a valuable individual (Ferreira, Hofmeyr, et al., 2014; Lee \& Roberts, 2016). Devaluation can occur through modifying the commodity to make it less valuable, such as staining a commodity intended for ornamental purposes, or by manipulating economic factors, such as supply and demand (Ferreira, Hofmeyr, et al., 2014; Haas \& Ferreira, 2015). For example, increasing the supply of a commodity can reduce its value because it becomes more accessible (Schneider, 2008). Proponents of devaluation strategies argue that the decrease in reward will discourage hunting and therefore protect species (Ferreira, Hofmeyr, et al., 2014). Alternatively, critics argue that devaluation strategies could unintentionally increase the commodity's market value, thereby encouraging illegal hunting (Ferreira, Hofmeyr, et al., 2014). Economic supply and demand theory states that when commodity supply declines, as occurs through devaluation, the market value of the commodity increases (Challender \& MacMillan, 2014).

To date, devaluation strategies have only been developed and applied to rhino populations that are threatened by illegal hunting. However, should these strategies prove 
effective at protecting rhino, many other illegally hunted species could benefit from similar strategies. While there are few examples of devaluation in the context of wildlife conservation, there are other examples of devaluation in society. Whilst very different, the logic behind devaluation is analogous to the decline in street crime, such as muggings and robberies, in response to people carrying less cash (Wright et al., 2014). As transactions have become increasing electronic and less cash based, criminals have been discouraged from engaging in street crimes (Wright et al., 2014). This resembles a devaluation strategy because criminal activity is discouraged as people carry less cash and petty crime becomes less profitable.

None of these strategies alone are likely to solve the IWT issue. Instead, a combination of strategies will be required (Challender, Wu, et al., 2014). Many the strategies discussed can be applied concurrently and are complementary. CITES can provide few examples of successful conservation all of which are due to a combination of strategies, usually community involvement and regulated trade (Challender \& MacMillan, 2014). Whilst strategies such as enforcement have been criticised, enforcement can be useful if implemented effectively. The challenge will be deciding when and where strategies and combinations of strategies should be implemented, and how intensively to implement them.

\subsection{Economics of the illegal wildlife trade}

As with all economic markets, factors that determine the scale (extent and total value) of IWT include consumer demand and commodity supply (Haas \& Ferreira, 2015). Manipulating these factors will change the risk-reward trade-off experienced by hunters. Reducing consumer demand can discourage hunting because with less consumers to purchase the commodity the market will diminish (Challender \& MacMillan, 2014). Reducing the market value of the commodity will also discourage hunters because their rewards are potentially reduced (Büscher \& Ramutsindela, 2016). Reducing commodity supply could also discourage hunting as locating animals becomes more difficult and the risk of capture or death becomes less tolerable. Cooney et al. (2017) provide a framework for combating the IWT. Their framework discusses four proposed strategies.

1. Increase the benefits that local communities receive from conservation. This often comprises community-based conservation strategies, and ensures that the local communities benefit financially from industries such as ecotourism (Cooney et al., 2017). 
Another controversial method of achieving this is through professional trophy hunting. Professional hunting in sub-Saharan Africa is a USD\$200 million per annum industry, and some countries such as Namibia and Zambia have successfully implemented programmes whereby a proportion of the revenue from these activities is received by local communities (Baker, 1997; Lewis \& Alpert, 1997; Njerekai \& Mabika, 2016). Through these strategies, local communities can learn to value wildlife and are motivated to preserve wildlife because of the benefits they receive (Cooney et al., 2017).

2. Decrease the costs that local communities face from living alongside wildlife. Human wildlife conflicts can negatively impact the livelihoods of people living alongside wildlife. Conflicts include personal security threats, livestock or crop loss, competition for resources and disease transmission (Cooney et al., 2017). Strategies to reduce humanwildlife conflicts are important as local communities become more tolerant of wildlife and more in favour of conservation (Woodroffe, Thirgood, \& Rabinowitz, 2005). However, this strategy is unlikely to succeed on its own, especially when wildlife commodities reach high values (Cooney et al., 2017).

\section{Reduce the benefits that local people and communities receive from of engaging in} the IWT. By reducing the benefits that communities receive from IWT, the risks of hunting illegally become less tolerable and can outweigh the benefits (Cooney et al., 2017). Devaluation strategies aim to reduce the rewards received by illegal hunters (Ferreira, Hofmeyr, et al., 2014). Another example of this is increasing the detection rates of devices such as snares used to entrap animals (Cooney et al., 2017). Again, while these strategies can be beneficial, they are unlikely to succeed independently and will require the support of other strategies (Cooney et al., 2017).

4. Increase costs that local communities face from engaging in the IWT. This is the most commonly used method as it includes increasing enforcement and punishments for illegal hunters (Duffy et al., 2016; Leader-Williams \& Milner-Gulland, 1993). While it is accepted that these strategies are important in preventing IWT engagement, excessive focus on these strategies has had negative consequences. (Lunstrum, 2014). These strategies should be implemented cautiously to prevent alienating local communities and should be augmented by other strategies (Challender \& MacMillan, 2014; Keane et al., 2008). 


\subsubsection{Economics of devaluation strategies}

Wildlife commodity devaluation strategies have been developed to protect species by discouraging illegal hunting (Ferreira, Hofmeyr, et al., 2014). However, economic supply and demand theory suggests that partial devaluation of a population could unintentionally encourage hunting (Challender \& MacMillan, 2014). Devaluing a high proportion of a large population is often not possible due to financial and logistical limitations (Ferreira, Hofmeyr, et al., 2014). Simple economic supply and demand theory states that when the supply of a commodity declines, the price of the remaining commodities will increase (Challender \& MacMillan, 2014; Harvey, 2016). This issue can be exacerbated when a commodity dichotomy is created, and when demand is price inelastic (Challender \& MacMillan, 2014; Ferreira, Hofmeyr, et al., 2014). Because these problems are common with wildlife commodities, reducing commodity supply, such as through devaluation strategies, could unintentionally incentivise illegal hunting of non-devalued animals in the population (Challender \& MacMillan, 2014; Harvey, 2016).

\section{Commodity Dichotomies}

When a devaluation strategy is applied to a population, a commodity dichotomy is created (Ferreira, Hofmeyr, et al., 2014). Removing the commodity from the animal results in animals that possess the commodity and those that do not (Ferreira, Hofmeyr, et al., 2014). Commodity modification results in animals that possess a valuable commodity and those whose commodities have been spoiled (Ferreira, Hofmeyr, et al., 2014). The demand for many wildlife commodities is largely based on cultural beliefs (Challender, Wu, et al., 2014). Commodities such as bear bile, rhino horn and pangolin scales are often used in TCM, despite no proven medicinal benefits (Ellis, 2013). When synthetic replicas of these commodities are created and introduced to the markets, cultures may perceive the replicas as inferior or useless (Dutton, Hepburn, \& Macdonald, 2011). This is even true for farming. Bear bile from farmed bears is perceived to have inferior medicinal qualities, with some people prepared to pay more for bile from wild bears (Dutton et al., 2011). Therefore, even though the true supply of the commodity may not have changed, a commodity dichotomy will have been created and the supply of the "superior" commodity is perceived to have reduced. 


\section{Price Inelastic Demand}

Price inelastic demand refers to a situation where the percentage change in demand divided by the percentage change in price is less than one. In other words, a change in the price of the commodity has minimal impact on demand (Challender \& MacMillan, 2014). This differs from the textbook supply and demand situation where increasing the price of a commodity will reduce demand and lowering the price will increase demand (Cartwright, 2011). In Figure 1.1, inelastic demand is represented by the slope of the demand curve $\left(\mathrm{D}^{1}\right)$ (Challender \& MacMillan, 2014). As the demand curve becomes steeper, demand becomes more price inelastic. This is analogous to a commodity becoming more valuable as is becomes rarer (Mason, Bulte, \& Horan, 2012). Even though wildlife devaluation will result in fewer fullvalue animals and a supply reduction (Figure 1.1, shift from $S^{1}$ to $S^{2}$ ), those that retain their full-value will be more valuable (Figure 1.1, price increase from $\mathrm{P}^{1}$ to $\mathrm{P}^{2}$ ) (Harvey, 2016). Subsequently, an unintended consequence of partial devaluation of a population is the increased value of the remaining commodities which could encourage hunting (Mason et al., 2012). 


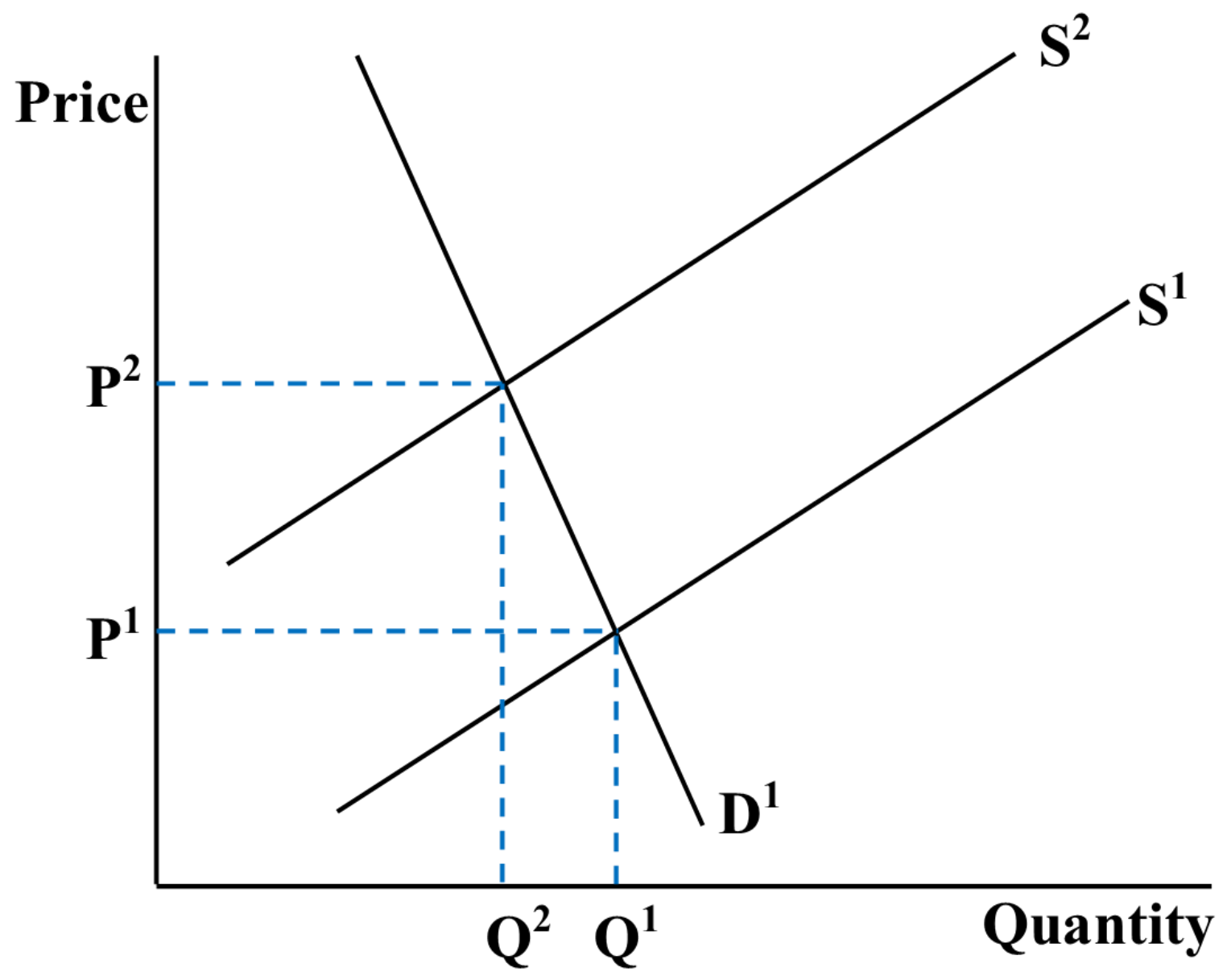

Figure 1.1. A graphical example of inelastic demand. The steeper the demand curve $\left(\mathrm{D}^{1}\right)$, the more inelastic the demand. Devaluation shifts the supply curve left $\left(S^{1}\right.$ to $\left.S^{2}\right)$ resulting in only a slight drop in quantity consumed $\left(Q^{1}\right.$ to $\left.Q^{2}\right)$, but a disproportionately large increase in price $\left(\mathrm{P}^{1}\right.$ to $\left.\mathrm{P}^{2}\right)$. Recreated from Challender and MacMillan (2014). 


\subsection{Behavioural modelling and games as models}

There are many biological, logistical and technological challenges associated with the devaluation of wildlife. Some of these challenges are related to the economics of devaluation. Others are species specific and related to the development and application of devaluation methods. However, these challenges could be mitigated or assist in determining how best to implement devaluation strategies. The more important uncertainty is whether devaluation influences hunter behaviour, and if so, how (Ferreira, Hofmeyr, et al., 2014). Greater knowledge is required on how illegal hunters respond to devaluation (Lindsey \& Taylor, 2011).

Until now, research on human behavioural responses to wildlife commodity devaluation strategies has been limited to theory and anecdotes. The theoretical concepts used have primarily been adopted from economics. Many aspects of the IWT are economic issues, suggesting that the human behaviours exhibited can be studied using economic theories such as game theory. Whilst this theoretical understanding has been useful in understanding and predicting human behavioural responses to devaluation strategies, the models used are limited by their assumptions. Within economics, the expected utility maximisation theory studies human decision making under risk, and assumes that humans behave rationally to maximise their utility (Myerson, 1991). However, this theory is criticised due to the observed behavioural anomalies exhibited by people (Kahneman \& Tversky, 1979). Understanding the behaviour of illegal hunters is challenging because of the clandestine nature of criminal activities and the dangers associated with studying criminal behaviour (Wheeler, 1976). Many behaviours exhibited by illegal hunters may seem irrational until the idiosyncrasies of the IWT are fully understood. For example, previous attempts to dehorn rhino have resulted in illegal hunters killing dehorned rhino (Martin, 1994). Conceptual economic modelling theories may overlook these complex and important aspects of hunter behaviour.

One way to overcome these limitations is through an applied examination of human behaviour. This is yet to be attempted in the context of wildlife commodity devaluation strategies. This research uses serious games as a method for studying human behaviour. While studying criminals is difficult, it is possible to study similar human behaviours in comparable but more benign circumstances, using games. Serious games are not commonly utilised as a research tool, particularly in a conservation context (Redpath et al., 2018; Vermillion et al., 2014). Nevertheless, they can be extremely useful. They expose the 
participants to simulations of real-world situations whilst overcoming ethical, logistical and health \& safety challenges (Garris, Ahlers, \& Driskell, 2002; Vermillion et al., 2017).

\subsection{Case study: Rhino conservation}

The plight of rhino species is an infamous example of the IWT. Rhino horn is valued at up to USD $\$ 65,000$ per kilogram (Biggs et al., 2013). Trading rhino horn internationally remains illegal despite some countries having legal domestic markets. The trade of rhino horn involves long and complex transportation networks with most horn sourced in Africa and sold in south-east Asia (Ayling, 2013). Three of the five remaining rhino species face the probability of extinction (Emslie, 2012b; van Strien, Manullang, et al., 2008; van Strien, Steinmetz, et al., 2008). Conservationists have proposed or attempted many conservation and hunting prevention strategies such as captive breeding, reintroductions, relocations (Kingdon et al., 2013), regulated legal trade (Biggs et al., 2013), farming (Murcott, 2017) and militarised security (Lunstrum, 2014). Wildlife commodity devaluation strategies are also being attempted (Ferreira, Hofmeyr, et al., 2014; Lindsey \& Taylor, 2011). This wide range of attempted conservation strategies makes the illegal hunting of rhino a particularly useful and interesting case study.

Rhino populations across the world have experienced three periods of significant decline. Hunting during the $19^{\text {th }}$ century caused a decline of the white rhino (Ceratotherium simum) to only 20-50 individuals (Emslie, 2012a). This decline was stopped, and white rhino recovered (Emslie, 2012a). A second decline occurred between 1970-1987 when 85\% of the world's rhino were killed (Ayling, 2013). This decline resulted in both species of African rhino being listed as an CITES Appendix I species in 1977, and the resultant international trade ban (Ayling, 2013; Biggs et al., 2013). Rhino species are now facing their third period of anthropogenic decline (Figure 1.2). Since 2007, rhino poaching has continually increased with over 1,000 rhino now being killed in South Africa annually (Emslie et al., 2016). This has increased from only 13 rhino in 2007 (Biggs et al., 2013). 


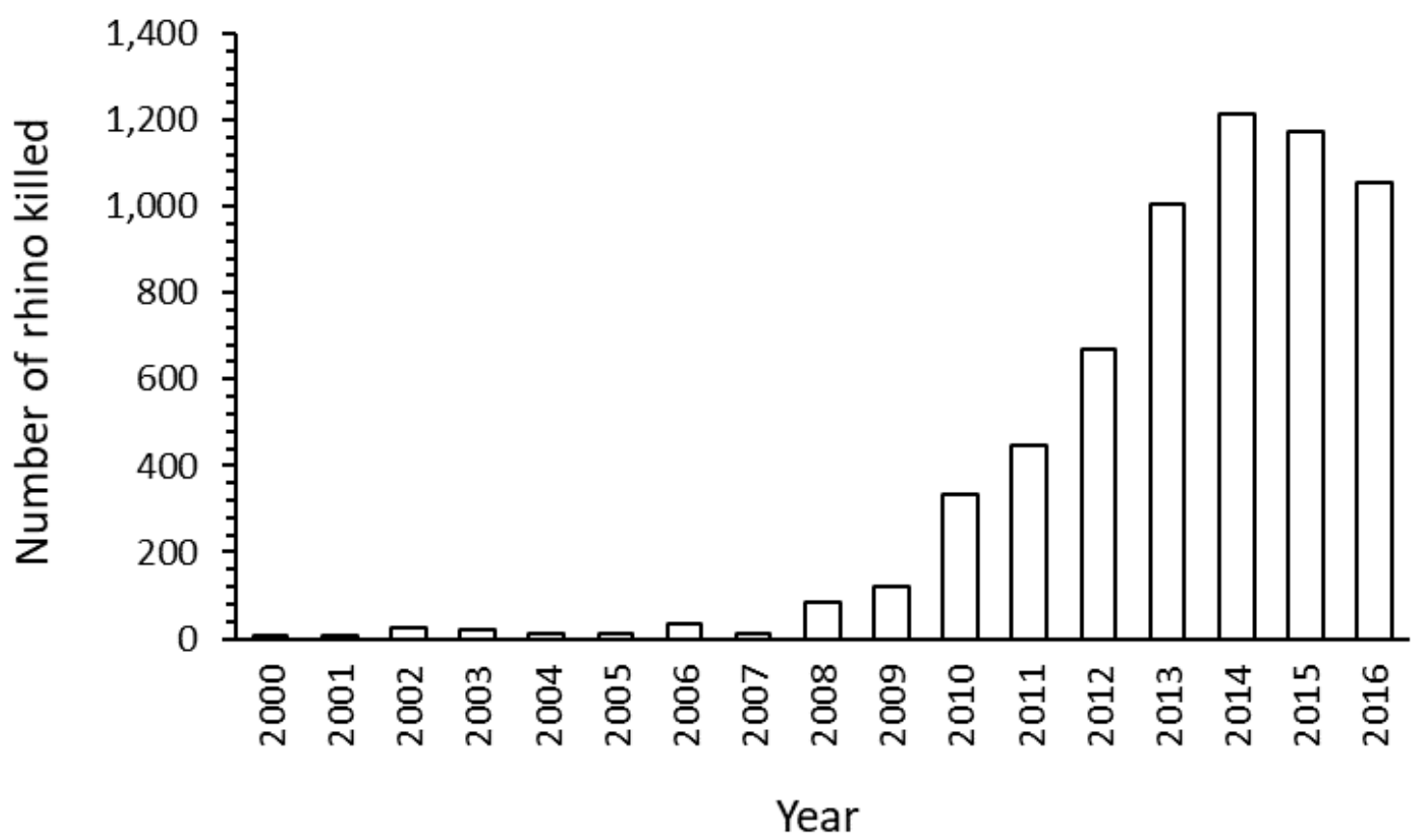

Figure 1.2. The escalation of illegal rhino hunting in South Africa between 2000 and 2016 (Biggs et al., 2013; Emslie et al., 2016; Trump, 2017). Apparent is the exponential increase in illegal hunting since 2007, before which time hunting levels were constantly low. 
Rhino horn is primarily trafficked to south-east Asia where it is falsely used to treat illnesses such as fevers, hangovers and cancer (Ayling, 2013). There is no scientific evidence that rhino horn is an effective treatment for any illness (Ayling, 2013). However, these medicinal uses have led to rhino horn being perceived as essential to life, and has resulted in its high demand and high value (Biggs et al., 2013). Due to its high value, a sub-market has been created whereby rhino horn is used for ornamental purposes as a status symbol (Ayling, 2013). These uses have caused demand for rhino horn to become price inelastic (Biggs et al., 2013).

Rhino populations are highly susceptible to illegal hunting due to their slow life history (Bennett, 2015). They have one of the longest gestation periods of any animal at 480 days (15-16 months) (Kingdon et al., 2013), they only produce a single calf per pregnancy and have long birth intervals of 2-2.5 years (Kingdon et al., 2013). Sexual maturity is reached at 7-8 years for females and 10-12 years for males (Kingdon et al., 2013). All these factors add to a low finite population growth rate ( $\lambda$; population growth per individual per unit time) (Bennett, 2015). Populations therefore take a long time to recover from declines, making them highly vulnerable to threats such as illegal hunting (Bennett, 2015).

\subsubsection{Rhinoceros species}

\section{White Rhinoceros (Ceratotherium simum)}

White rhino are the most common species with an estimated population of 20,378 in 2015, and an IUCN classification of Near Threatened (Figure 1.3) (Emslie, 2012a; Emslie et al., 2016). Two sub-species of white rhino exist. The southern white rhino (Ceratotherium simum simum) with a population of 17,460 individuals is classified as Near Threatened (Emslie et al., 2016). Their historical range includes Namibia, Botswana, Zimbabwe, Mozambique, South Africa and Swaziland (Emslie, 2012a). Only South Africa retains an original population, while the other countries have received reintroductions following local extinction from over-kill (Emslie, 2012a). Translocations to countries outside of the historical range have also occurred to Kenya, Uganda and Zambia (Emslie, 2012a). The northern white rhino (Ceratotherium simum cottoni) became functionally extinct when the last surviving male, Sudan, died in March 2018 (Gibbens, 2018). Two females survive at the Ol Pejeta Conservancy in Kenya (Gibbens, 2018). Their historical range included Uganda, Chad, Sudan, the Central African Republic and Democratic Republic of the Congo (Emslie, 2012a). 


\section{Black Rhinoceros (Diceros bicornis)}

Black rhino is the most threatened species of African rhino and is classified as Critically Endangered (Emslie, 2012b). During the $20^{\text {th }}$ century, black rhino were the most common species of rhino (Emslie, 2012b). However, since 1960 black rhino numbers have decreased by $97.6 \%$ (Emslie, 2012b). As of 2015, only an estimated 5,250 individuals remain across three sub-species (Emslie et al., 2016). The south-western black rhino (Diceros bicornis bicornis) is classified as Vulnerable with an estimated population of 2,200 (Emslie et al., 2016). The southern-central black rhino (Diceros bicornis minor) is classified as Critically Endangered with an estimated population of 2,164 (Emslie et al., 2016). The eastern black rhino (Diceros bicornis michaeli) is classified as Critically Endangered with an estimated population of 886 (Emslie et al., 2016). The western black rhino (Diceros bicornis longipes) was declared extinct as recently as November 2011. Despite the small population size of this species, they are relatively widespread across Africa with many small populations (Emslie, 2012b). The strongholds of this species are South Africa and Namibia, with small populations present in Kenya, Zimbabwe, Tanzania and Botswana (Emslie, 2012b).

\section{Indian Rhinoceros (Rhinoceros unicornis)}

The Indian rhino (also known as the greater one-horned rhinoceros) is the most common Asian species of rhino. It had an estimated population of 3,264 individuals in 2015 and is classified as Vulnerable (Emslie et al., 2016; Talukdar et al., 2008). Historically it was found across Pakistan and northern India, but the current population is restricted to a few heavily protected reserves in India and Nepal (Talukdar et al., 2008).

\section{Sumatran Rhinoceros (Dicerorhinus sumatrensis)}

The Sumatran rhino is considered Critically Endangered with only 76 individuals in 2015 (Emslie et al., 2016; van Strien, Manullang, et al., 2008). Historically this species was found in the Himalayan region of Bhutan and Southern China, through south-east Asia to the Sumatran and Bornean islands of Indonesia (van Strien, Manullang, et al., 2008). Today the species is only found in very small populations in Indonesia, with unconfirmed populations in Malaysia and Myanmar (van Strien, Manullang, et al., 2008). Despite the small numbers, three sub-species remain (van Strien, Manullang, et al., 2008). Dicerorhinus sumatrensis lasiotis is probably extinct, but a small population potentially persists in Myanmar (van Strien, Manullang, et al., 2008). Dicerorhinus sumatrensis harrissoni is believed to have only 
3 individuals surviving in Indonesia, whilst Dicerorhinus sumatrensis sumatrensis, is believed to have 73 individuals surviving in Indonesia (van Strien, Manullang, et al., 2008).

\section{Javan Rhinoceros (Rhinoceros sondaicus)}

The Javan rhino (also known as the lesser one-horned rhinoceros) is the most threatened rhino species. Rhinoceros sondaicus sondaicus is the only extant sub-species numbering 63 with an IUCN classification of Critically Endangered (Emslie et al., 2016; van Strien, Steinmetz, et al., 2008). Historically this species could be found from Bangladesh through to Bhutan and Southern China, south through south-east Asia to the Sumatran and Javan islands of Indonesia (van Strien, Steinmetz, et al., 2008). Today the species is only found in a small part of the Indonesian island of Java (van Strien, Steinmetz, et al., 2008). 

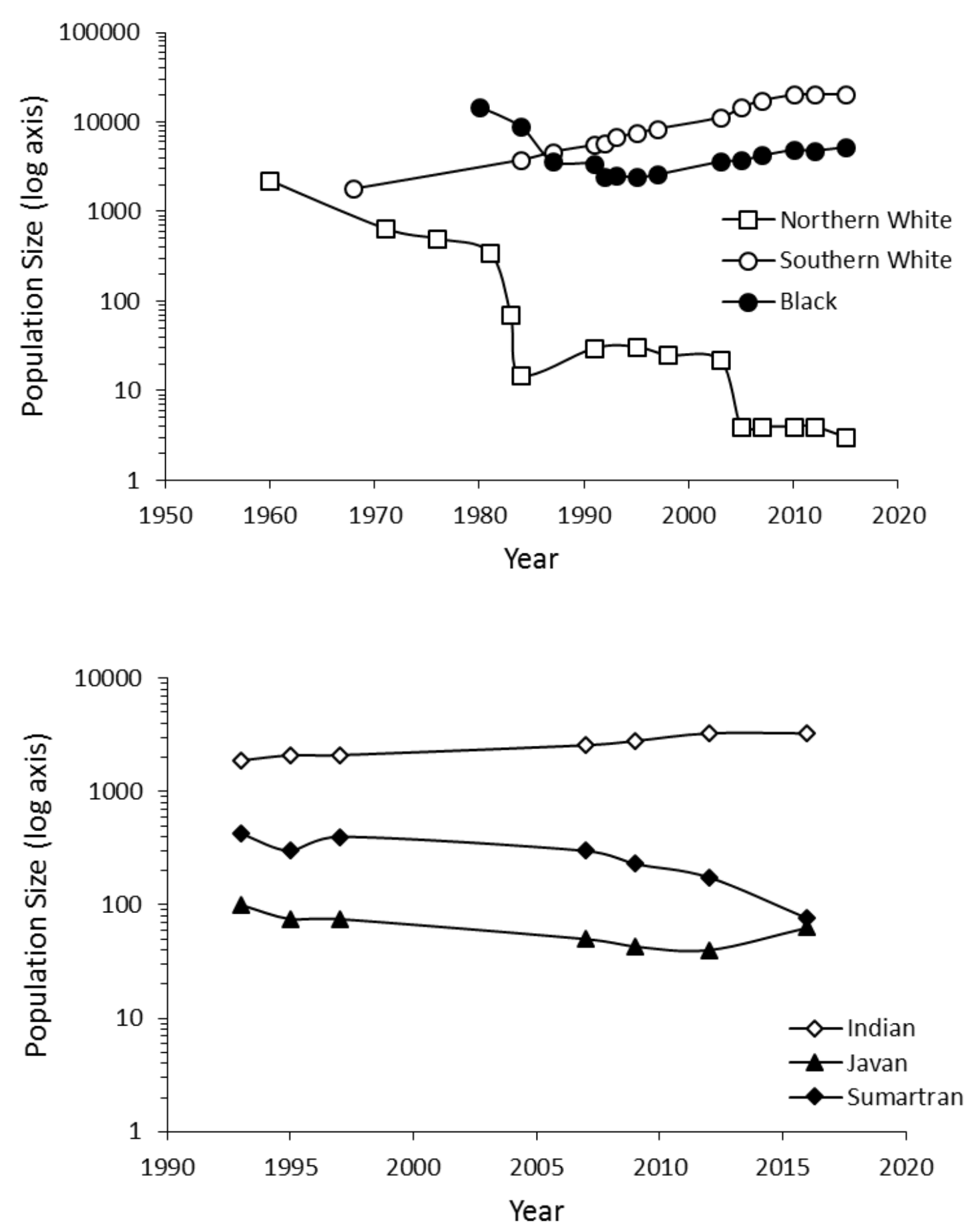

Figure 1.3. Trends in rhinoceros numbers for (a) African species and (b) Asian species (Emslie, 2004; Emslie \& Brooks, 1999; Emslie et al., 2007; Emslie, Milliken, \& Talukdar, 2013; Emslie et al., 2016; Foose \& van Strien, 1997; Milliken, Emslie, \& Talukdar, 2009). The African Northern and Southern white rhino sub-species are graphed separately. 


\subsubsection{Devaluing rhino}

Wildlife commodity devaluation strategies are a topical hunting prevention strategy. Devaluation strategies are currently only applied to populations of rhino that are threatened by illegal hunting. Multiple methods of devaluing rhino have been proposed, and some applied. If rhino devaluation strategies can be proven effective, many other species could benefit from having species-specific devaluation methods developed and applied to protect them from illegal hunting.

Horn removal as a method of devaluation was first attempted on a large scale in 1989 (Milner-Gulland et al., 1992). This involves tranquilizing the rhino and removing the horn above the nerve and germinal layer to prevent infection and deformed regrowth (Figure 1.4) (Lindsey \& Taylor, 2011). While dehorning is expected to discourage hunters from killing the rhino, the technique has constraints and limits. Rhino horns regrow meaning that valuable horn will replenish. It is estimated that dehorning must be repeated every 1.3 years for the method to successfully discourage illegal hunters (Milner-Gulland et al., 1992). Such frequent animal immobilisation is probably unachievable, especially for large populations (Büscher \& Ramutsindela, 2016). Horn removal must occur approximately $5 \mathrm{~cm}$ and $7 \mathrm{~cm}$ above the base of the posterior and anterior horns respectively, to prevent infection and damage to the nerve and germinal layers (Lindsey \& Taylor, 2011). This means that a stump of valuable horn remains which can still be illegally harvested. Evidence from previous dehorning programs suggests that hunters are still be motivated to kill dehorned rhino and harvest the small amount of valuable horn that remains or has regrown (Milner-Gulland, LeaderWilliams, \& Beddington, 1994). There is also uncertainty over whether dehorning is detrimental to rhino survival (J. Berger \& Cunningham, 1994). Calf mortality is potentially higher among calves whose mothers have been dehorned because the mother's reduced ability to protect her calf from predators (J. Berger \& Cunningham, 1994), and repeated tranquilisation can negatively impact female rhino fertility (Alibhai, Jewell, \& Towindo, 2001). Also, as more of the population is dehorned, the costs of locating, tranquilising and dehorning the remaining animals increase as they become more difficult to locate (Ferreira, Hofmeyr, et al., 2014). 


\section{This content is unavailable.}

\section{Please consult the print version for access.}

Figure 1.4. The dehorning process. Wildlife managers remove a horn with a chainsaw from a tranquilized rhino (Wildlife ACT, 2018). 
Rhino horn can also be modified or spoiled. A method has been developed by the Rhino Rescue Project (Rhino Rescue Project, n.d.-a) to infuse rhino horn with a toxin and coloured dye (Figure 1.5) (Ferreira, Hofmeyr, et al., 2014). This is promoted as less invasive than dehorning, as the horn is not removed (Ferreira, Hofmeyr, et al., 2014). It involves drilling in to the horn and inserting probes which infuse an inedible toxin under high pressure throughout the horn (Ferreira, Hofmeyr, et al., 2014). The toxin used is an ectoparisiticide which makes the horn unsuitable for medicinal consumption (Ferreira, Hofmeyr, et al., 2014). This method of devaluation assumes that because rhino horn is made up of many tubules (similar to a sponge), a liquid could be infused through the horn (Ferreira, Hofmeyr, et al., 2014). A coloured dye is also infused to make the horn unsuitable for ornamental use, and to alert hunters to the commodity's devalued status (Büscher \& Ramutsindela, 2016). However, as with dehorning, there are limitations. Ferreira, Hofmeyr, et al. (2014) discuss how the infusion process is ineffective at distributing the toxin and dye through the entire horn, and therefore some valuable horn can be salvaged from an infused horn. This is because rhino horn is less porous than initially assumed (Boy et al., 2015; Ferreira, Hofmeyr, et al., 2014). The procedure costs over USD $\$ 1,000$ per animal, but these costs increase as more animals are treated and it becomes more difficult to locate animals that have not been devalued (Ferreira, Hofmeyr, et al., 2014). Additionally, the fact that horns grow throughout the animals life means that after infusion, unspoilt horn is continuously replenished (MilnerGulland et al., 1992). 


\section{This content is unavailable.}

\section{Please consult the print version for access.}

Figure 1.5. The horn infusion process. Holes are drilled in the horn and high pressure infusion probes are inserted into the holes to infuse a toxin into the horn (Rhino Rescue Project, n.d.-b). 
Devaluation can also be achieved by manufacturing and releasing synthetic replicas of wildlife commodities to the market to meet demand. Synthetic replicas can also be used to increase supply and drive the price down (Crookes, 2017). Synthetic rhino horn is being developed with the goal of reducing hunting pressure on wild populations of rhino (Crookes, 2017). Synthetic substitutes are also being developed for other wildlife commodities. A synthetic substitute for bear bile is being developed by Kaibao Pharmaceuticals (Livingstone, Gomez, \& Bouhuys, 2018). Bear bile is a highly sought-after commodity across Asia for use in TCM (Feng et al., 2009). Bears are housed in inhumane bile factories with bile being continuously extracted from them and it is hoped that a synthetic substitute for bear bile will decrease the industry (Livingstone et al., 2018). However, concerns exist around the effectiveness of synthetic substitutes because the synthetic and farmed replicas can be considered inferior compared to a true commodity from a wild animal (Dutton et al., 2011).

\subsubsection{Outcomes of previous devaluation attempts}

Dehorning was first attempted in Namibia the late-1980s, and Zimbabwe followed soon after in the early-1990's. Modelling studies from as early as 1992 suggested that on its own, dehorning would not be an effective hunting reduction strategy (Milner-Gulland et al., 1992). Many attempts to dehorn rhino populations have proved to be unsuccessful at deterring illegal hunters and protecting rhino.

The first attempt at dehorning in Namibia proved successful (Lindsey \& Taylor, 2011). However, this programme was coupled with improved security and conservation funding (Lindsey \& Taylor, 2011). Attempts to dehorn in Zimbabwe and Swaziland also proved effective, but these programmes were coupled with translocations of rhino to secure sanctuaries and parks away from park and country borders (Lindsey \& Taylor, 2011). In Zimbabwe, dehorning succeeded in reducing mortality rates from $14.1 \%$ in horned populations, to $9.1 \%$ in dehorned populations (du Toit, 2011). Other small-scale attempts to dehorn in Mozambique and South Africa suggest some success, but these conclusions are tentative (Lindsey \& Taylor, 2011).

Rhino dehorning was attempted in Hwange National Park, Zimbabwe in 1992 (Lindsey \& Taylor, 2011). Unfortunately, 12-18 months after the dehorning, the Department of National Parks and Wild Life Management suffered a budget reduction that resulted in many 
parks having to reduce their security for a period of six months (Lindsey \& Taylor, 2011; Milner-Gulland et al., 1994). Subsequently, nearly all of the dehorned rhino were killed (du Toit \& Anderson, 2013; Lindsey \& Taylor, 2011). This trend was mirrored in other Zimbabwean parks (Matobo National Park, Matusadona National Park, Chipinge Safari Area and Sinamatela) all of which dehorned a large proportion of their rhino population, but still experienced significant rhino losses due to poor security (Lindsey \& Taylor, 2011). The Chiredzi River Conservancy, another reserve with poor security, lost 27 of its 29 rhinos, all of which were dehorned (Du Toit, 2011). In 2011, the Save Valley Conservancy dehorned six rhino all of which were subsequently killed (Lindsey \& Taylor, 2011). These outcomes suggest that security is more effective than devaluation at deterring hunters and protecting rhino, and that devaluation is not an effective alternative to security. It raises the question as to why devaluation did not protect rhino? It also raises the possibility that illegal hunters respond to devaluation in an unexpected manner by continuing to hunt and by killing devalued animals.

\subsection{Research aims and hypotheses}

The primary aim of this research is to describe and measure the response of human behaviour to a commodity devaluation strategy. Much of the theoretical and anecdotal evidence on wildlife commodity devaluation suggests that the strategies are not successful. It is hypothesised that the results of this study will confirm these theoretical and anecdotal conclusions.

I also aim to provide insight into why devaluation strategies have been unsuccessful. It is hypothesised that the reason for their failure is that a high threshold of devaluation is required. Empirically testing this threshold is necessary but has never been attempted. Theory predicts the threshold to be at least $50 \%$ because a hunter then has a greater chance of locating a devalued animal as opposed to a valuable animal (Ferreira, Hofmeyr, et al., 2014). However, Milner-Gulland (1999) suggest that dehorning 50\% of a population would not be sufficient, and that devaluation will become more effective as the level of devaluation increases.

This research aims to show how people respond differently to devaluation and risk. It is hypothesised that risk will be a more effective deterrent than devaluation. 
It also aims to demonstrate how manipulating commodity value (devaluation) or risk (security) in one place may impact hunting in other places, thus illustrating the need for strategies to be coordinated across all vulnerable populations.

This research aims to examine how the magnitude of devaluation (how much of the value is lost during the devaluation process) influences hunting behaviour. It is expected that as the magnitude of devaluation increases, towards the commodity being rendered worthless, it is less likely to be harvested.

Finally, this research aims to demonstrate how games can be applied as a useful method of studying human behaviour. Games are expected provide a greater understanding of the irrational behaviours and behavioural nuances that are overlooked by theory.

\subsection{Thesis structure}

This thesis incorporates three research methods - two games and an online survey. Rather than structuring the thesis in separate research chapters, the thesis follows the standard IMRaD (introduction, methods, results and discussion) format. Although both games and the survey are different, their development, methods, results and discussion are closely related. Presenting and discussing the games and survey, and their outcomes, together assists in making comparisons.

Chapter 2 discusses the theories (primarily economic) used to study human behaviour, particularly those used to study human behavioural responses to devaluation strategies. It discusses the use of games as a research tool, previous research that has used similar methods, and what is required for a game to be an effective research tool. I was involved in several pilot studies of the games conducted in the years leading up to this thesis. They were critical to the development of the game methodologies used in this thesis. Thus, a brief description of these pilot studies is presented in this Chapter 2 to illustrate how they informed the development of the games.

Chapter 3 discusses the research methods used. This chapter is divided into sections that separately explain the methodologies of the games and the survey. Chapter 4 presents the results of the games conducted. Again, this chapter is divided into sections that separately explain the results of the games and the survey. Chapter 5 forms a combined discussion of the project. As well as discussing the results, this chapter discusses how to apply the research to the real-world, and ways in which the games could be enhanced. It also discusses the 
usefulness of using games as a research tool, and why experimental games should be used more frequently, particularly in difficult-to-study disciplines. 


\section{Theory and Games}

\subsection{Behavioural theory and modelling}

While there are many drivers of the illegal wildlife trade (IWT), such as a variety of social and cultural influences, economic factors also influence the system significantly (Haas \& Ferreira, 2015). Economic factors such as supply and demand, together with risk and reward, are important and effective regulators of commodity price, and therefore the trading relationships and market systems involved (Haas \& Ferreira, 2015). It is for this reason that many hunting-prevention strategies attempt to manipulate economic factors.

Illegal hunting can be discouraged by increasing the relative cost of the activity (Tietenberg, 2006). Militarised security increases the risk hunters' experience of being arrested or killed (Lunstrum, 2014). Devaluation reduces the probability of locating a valuable animal and reduces the expected value of animals found, thereby increasing the riskreward ratio (du Toit \& Anderson, 2013; Ferreira, Hofmeyr, et al., 2014). Due to the economic character of the IWT, a theoretical understanding exists of how illegal hunter behaviour might respond to devaluation strategies. Most of this theory comes from behavioural economics and microeconomics in the form of conceptual models for human behaviour. Examples of the conceptual modelling theories that have been used to study human behaviours in conservation include game theory, rational choice theory and decision theory.

\subsubsection{Game theory}

Game theory is the study of competitive situations and the optimal decision making of players in these situations (Besanko \& Braeutigam, 2005). While game theory is an economic theory, it has much wider applications. Game theory is one of many equilibrium analyses used to determine what behaviours individuals will exhibit and what outcomes will follow (Kreps, 1990). In game theory, individuals rarely determine their own fate because their actions are dependent on the actions of others (Besanko \& Braeutigam, 2005; Kreps, 1990). In the simplest games, two players compete by each making a single decision simultaneously (Besanko \& Braeutigam, 2005). In the context of wildlife devaluation strategies, the two actors are usually the wildlife manager and the illegal hunter. More complex games involve additional players, decisions with more than two possible actions, plays repeated more than 
once, sequential rather than just simultaneous actions, and strategic behaviours (Besanko \& Braeutigam, 2005).

Game theory modelling is frequently applied to conservation issues, most commonly illegal or over-harvesting (Colyvan, Justus, \& Regan, 2011). For example, Gibson and Marks (1995) used game theory to analyse failed community-based wildlife protection and management in Zambia. This application of game theory modelled the actions of illegal hunters and local scouts to show that community-based initiatives failed because inaccurate assumptions were made about hunter behaviour (Gibson \& Marks, 1995). Mesterton-Gibbons and Milner-Gulland (1998) also used game theory to analyse a community-based wildlife management programme. Under Zimbabwe's CAMPFIRE programme, local residents must decide whether to hunt illegally or enforce hunting laws, and incentives not to hunt are provided to locals (Mesterton-Gibbons \& Milner-Gulland, 1998). Using game theory as a framework, they found that as well as providing incentives to refrain from illegal hunting, it is also important to provide incentives for residents to engage in policing (Mesterton-Gibbons \& Milner-Gulland, 1998). For a self-monitoring strategy to work, each individual must receive a payment that exceeds the opportunity cost of monitoring (Mesterton-Gibbons \& Milner-Gulland, 1998).

Game theory is not limited to modelling competitive systems, but also cooperative systems such as how countries can cooperate to manage and conserve threatened fish stocks. Fisheries stocks are often characterised as a common-pool resource, and are therefore susceptible to the Tragedy of the Commons (Hardin, 1968; Sumaila, 1999). Game theory modelling has demonstrated why a non-cooperative approach to managing open access common-pool resources, such as fisheries, is unsustainable (Sumaila, 1999). These findings have been used to help develop mutually beneficial management agreements for many fish stocks, including Northeast Atlantic cod and the Southern Bluefin tuna (Sumaila, 1999).

Game theory has also been applied specifically to study the response of hunter behaviour to wildlife commodity devaluation strategies. Lee and Roberts (2016) use game theory to model the competitive relationship between the illegal hunter and the wildlife manager. These models suggest that the level of devaluation required for the strategy to succeed is high (Lee \& Roberts, 2016). This means that it will be financially and logistically difficult for wildlife manages to effectively implement devaluation strategies (Lee \& Roberts, 
2016). These models for hunter-guard behaviour from game theory provide a guide to the application of devaluation strategies and provide predictions to test.

\subsubsection{Rational choice theory}

Within economics, the rational choice model is the standard economic decision-making model (Just, 2014). The rational choice model assumes that humans make choices based on what they perceive to be best for their wellbeing based on the information they have available to them, and any other decision-making constraints (Just, 2014). The rational choice model is the most common method of conducting economic analyses of human behaviour (Duffy et al., 2016).

The rational choice model is able to model decision-making under risk (Just, 2014). Risk is defined as a situation whereby a decision must be made prior to knowing the pay-outs of other actions (Just, 2014). Models of rational choice have previously been applied to studying the human behaviour of illegal hunting. Milner-Gulland and Leader-Williams (1992) used rational choice theory to model the rewards, chance of detection and penalties faced by illegal hunters in Zambia. Their models showed that the probability of detection has a large impact on whether an illegal hunter decides to hunt, and fixed penalties are less effective at deterring illegal hunters than penalties based on the illegal hunter's output (e.g. the number of trophies confiscated) (Milner-Gulland \& Leader-Williams, 1992). Rational choice theory is yet to be applied to the study of human behaviour in response to devaluation strategies.

\subsubsection{Decision theory}

Decision theory is the study of goal-directed behaviours in situations where multiple behavioural options are possible (Hansson, 2011). Decision theory has a heavy grounding in statistics, and close ties to economics shown through its similarities with game theory (Myerson, 1991). An important difference between these two concepts - decision theory and game theory - is that game theory treats several actors as competing equals that influence each other's decisions (Hansson, 2011). Decision theory focusses on a single decision maker and the actions of other actors are treated as external, akin to natural events (Hansson, 2011). For example, if illegal hunter behaviour is being modelled, the actions of the wildlife manager would be treated as external, rather than as a second decision maker. Decision 
theory focuses on studying the outcome of decisions under uncertainty caused by multiple behavioural paths that might reach a specific goal (Hansson, 2011). This involves determining the decision maker's alternative decisions, the possible outcomes of each alternative decision, the values for each of those outcomes, and any extraneous factors that could affect the outcome (Hansson, 2011).

Decision theory is often aided by statistics (statistical decision theory) whereby statistical knowledge or probabilities are assigned to each decision (J. O. Berger, 1985). This statistical knowledge explains some of the uncertainty involved in the decision, thereby assisting the decision making process (J. O. Berger, 1985). Decision theory has been applied to conservation for many species such as the Sumatran rhino (Regan et al., 2005). Regan et al. (2005) used decision theory to analyse the decision problem of how to manage this critically endangered species. Decision theory was used to analyse the management options of captive breeding, relocation or the formation of a new reserve in response to hunting, habitat destruction, and demographic and disease threats (Regan et al., 2005). Under low uncertainty, captive breeding was the best strategy, but as uncertainty increased, forming a new reserve became optimal (Regan et al., 2005). Decision theory has not been applied to the study of wildlife commodity devaluation strategies.

\subsection{Modelling hunter behaviour under devaluation strategies}

The devaluation of rhino has been considered a theoretical game between hunters and wildlife managers. Previous research has used game theory to model hunter responses to devaluation strategies applied by wildlife managers (Glynatsi, Knight, \& Lee, 2018; Lee \& Roberts, 2016). The theoretical models of Lee and Roberts (2016) and Glynatsi et al. (2018) consider that a wildlife manager can either devalue a proportion of their rhino population or not, and that illegal hunters can either behave selectively (only kill animals that have not been devalued) or indiscriminately (kill all animals encountered) (Glynatsi et al., 2018; Lee \& Roberts, 2016). Their models assume that managers do not select either devaluation or security but a combination of both, and that managers would ideally devalue the least number of rhino possible while ensuring the protection of the population. The results of these studies suggest that high proportions of a population must be devalued before hunting is adequately deterred, and that the higher the level of devaluation, the more effective the strategy will be (Glynatsi et al., 2018; Lee \& Roberts, 2016; Milner-Gulland et al., 1992). 
The high levels of devaluation that theoretical studies suggest is necessary can be explained by several factors. Low rates of hunter detection and lenient penalties mean that the risks associated with illegal hunting are perceived as low (Sollund, 2016). The extreme value of rhino horn means that illegal hunters have a high risk tolerance (Sollund, 2016).

Devaluation is also an expensive and time-consuming process, and becomes more expensive and time-consuming as more of the population is treated and the remaining animals become more difficult to locate (Ferreira, Hofmeyr, et al., 2014). Achieving high levels of devaluation will exhaust resources (e.g. finances and time) that are sometimes acquired from elsewhere, such as enforcement (Büscher \& Ramutsindela, 2016). Therefore, achieving the high levels of devaluation that theoretical models have suggested will be necessary is unlikely.

\subsection{Games as models of human behaviour}

Games are defined as "a competitive activity involving skill, chance, or endurance on the part of two or more persons who play according to a set of rules, usually for their own amusement or for that of spectators" (Game [Def. 1], n.d.). However, as games have become increasingly used for non-entertainment purposes, the term "serious games" has been coined. Serious games are defined as games that are used for purposes other than entertainment (Vermillion et al., 2017). They can be used for education, training, social change, simulation, and advertising (Almeida et al., 2017; Susi, Johannesson, \& Backlund, 2007a). For these applications, serious games have been applied to many different fields including medicine, military and defence, sports (e.g. Formula 1) and aerospace (Almeida et al., 2017). The definition of serious games is often limited to digital games, but for this research and thesis, the definition is extended to include any game format.

Another application of serious games is for research (Vermillion et al., 2017). Game theory defines a game as "a model of a strategic situation in which the outcome of the actions of an individual also depends on the actions chosen by others" (Redpath et al., 2015, p. 415). Games provide a method of analysing conflict and cooperative behaviour, and how changes to a system of relationships impact those relationships and outcomes (Relyea \& Hoverman, 2006). Games can also provide a more affordable and equally effective replacement for social science research, especially when the research requires investigating real-world systems (Chesney, Chuah, \& Hoffmann, 2009). Games allow for participants to engage in complex behaviours. This means games can offer a mechanism for researching complex subject matters, such as decision-making, within disciplines such as psychology and economics 
(Garris et al., 2002; Järvelä et al., 2014a). However, serious games (particularly non-digital serious games) are underutilised as a research tool, and there exists little guidance on how to choose, setup and apply games to research (Järvelä et al., 2014b; Vermillion et al., 2014).

A model is defined as "a simplified representation or description of a system or complex entity" (Model [Def. 9], n.d.). Throughout this chapter and thesis, the phrase conceptual model is used. By this, I mean theories such as game theory, rational choice theory and decision theory. The conceptual models can be represented by empirical models which describe mathematical relationships between their parts (parameters) (Clarke \& Primo, 2012). Empirical models can be evaluated evidentially using Information Theoretic approaches to hypothesis testing, selection and inference. Conceptual theories, like game theory, have been used to develop hypotheses and make predictions about human behaviour. However, such conceptual models are limited in their ability to model complex human behaviours such as irrational behaviours, behavioural nuances and interactions between people (Redpath et al., 2018). Tests of empirical models using serious games could therefore be an important advance on conceptual models. There have been few attempts to develop applied methods of studying these complex systems and human behaviours.

Garris et al. (2002) use the terminology simulation rather than serious game, potentially because the article were published before the term serious game was coined. Garris et al. (2002) describe a simulation as an operating model representing a real-world system, and that this characteristic is the key distinction between games and simulations. The description provided by Garris et al. (2002) is very similar to the definition of a conceptual model - a simplified representation of a system (Model [Def. 9], n.d.). Garris et al. (2002) define the key characteristics of simulations as:

1. a representation of a real-world system that can incorporate some characteristics of reality,

2. including rules (as games do) and strategies that allow for flexible activity and behaviours to be exhibited, and

3. reducing the costs to participants to protect them from the more severe consequences of reality. 


\subsubsection{Use of games in conservation}

Games are being increasingly utilised as an innovative research method. This is largely driven by technological advancements and electronic devices becoming more readily accessible (Sandbrook, Adams, \& Monteferri, 2015). There are three primary ways in which games can be utilised in conservation (Sandbrook et al., 2015). Games such as MyConservationPark require players to manage their own virtual nature reserve (Sandbrook et al., 2015). Through this, players are taught about conservation principles, and can lead to a greater connection with nature and potentially positive environmental behaviours (Sandbrook et al., 2015). Games can also be designed to operate as a conservation fundraising mechanism (Sandbrook et al., 2015). The game Sims Social enabled gamers to purchase a panda within the game for a donation of $\$ 1.75$, which generated $\$ 40,000$ for WWF (Sandbrook et al., 2015). The revenue from such games can be invested into conservation (Sandbrook et al., 2015). Games can also be used as a citizen science tool for research, wildlife monitoring and planning. Mobile applications such as $e$ Bird provide a platform for people to lodge observations of nature which provides information to researchers about species distribution and abundance (Sullivan et al., 2014). These applications can also include a game component allowing users to compete against each other (Sandbrook et al., 2015).

Currently, the use of games for conservation research has not extended to the analysis of conservation conflicts. Additionally, using games to study human behaviour is largely limited to the theory of such games, not their practice (Redpath et al., 2018). However, the possibility to draw upon or modify game platforms - such as Pokémon Go - to simulate realworld conservation scenarios has been considered (Dorward et al., 2017). While these games often incorporate a competitive element, these elements are not well developed. Developing the competitive and cooperative elements of these games could advance the testing of concepts and behaviours in complex socio-economic systems, such as the IWT.

\subsubsection{Benefits of using games to study human behaviour}

Despite the benefits that conceptual modelling has provided to scientific research, these methods have one major limitation. As mentioned, many economic theories (e.g. game theory) assume that people act rationally to maximise their expected utility. Within economics, the concept of utility is used as a measure of the usefulness, happiness or value that a person receives from a good, service or behaviour (Cartwright, 2011). The utility 
maximisation hypothesis asserts that if a person is rational, then they attempt to maximise their utility through their actions or behaviours (Myerson, 1991). Many of the theories used in economics (e.g. game theory and rational choice theory) assume that the individuals behave to maximise their utility (Just, 2014; Myerson, 1991). However, this theory has come under scrutiny due to observed behavioural anomalies exhibited by people (Kahneman \& Tversky, 1979). Illegal hunters can exhibit seemingly irrational behaviours. For example, if a hunter kills a devalued animal to prevent locating it again (Lindsey \& Taylor, 2011), and increase their chances of locating a full-value animal, a rational decision has been made with the intention of increasing their utility. However, if a hunter kills a devalued animal in frustration (Martin, 1994), that may be considered an irrational decision because an unnecessary additional risk has been taken with no intention of increasing their utility.

Serious games provide a way of testing for irrational and rational behaviours. IWT networks involve many participants including wildlife managers, guards, illegal hunters and buyers which can result in complex and sometimes unexpected interactions occurring (Nijman, 2010). Corruption is known to occur at many levels of the IWT system (Bennett, 2015). Other forms of interaction might include cooperation between hunters, or hunters cooperating with guards. Conceptual modelling techniques are limited in their ability to explore these aspects of the IWT system (Redpath et al., 2018). Understanding these behaviours and interactions is important when trying to understand the complex IWT system. Applied experimental methods, such as serious games, may enable modelling of these behaviours and interactions.

Studying the rationales behind people's behaviours, not just the behaviours themselves, is also possible. This is achieved through qualitative research methods, such as surveying participants about their behaviour and participation after the game (Redpath et al., 2018). For any single behaviour, there are numerous potential rationales as to why that behaviour was exhibited. As discussed above, anecdotal evidence suggests that hunters may kill a devalued animal for many different reasons. Understanding why a behaviour is exhibited is important, especially if the goal of the research is to advise or design behaviour change interventions.

Games also allow for the replication and study of contexts and systems that in reality would be difficult or unethical to observe (Redpath et al., 2018). An example of this is observing and researching criminal behaviour. Criminal relationships and behaviours are difficult to investigate and understand due to the clandestine nature of the activities, and the 
dangers involved in associating with criminal systems and people (Wheeler, 1976). Therefore, understanding the IWT system and the behaviours of those involved has proved difficult. However, it may be possible to examine these behaviours through other people (non-criminals) in similar but more benign circumstances, using games. There will be differences between the behaviours of criminals and non-criminals, and differences between the real-world systems and the games designed to replicate these systems. However, if games prove effective at eliciting behaviours from non-criminals that are similar to those exhibited by criminals in the real-world, games could provide an innovative method of researching criminal systems and behaviours.

Finally, experiments and research though game-like conditions can provide highly accurate results (Washburn, 2003). Surveys and written accounts are limited in their ability to study complex systems because the respondent's self-reports are interpretations (Vermillion et al., 2017). Games provide a more open-ended approach as the participant experiences the scenario, instead of reading it, and their decision-making and behaviour can be observed through game play. Compared to surveys and written accounts, game-like conditions can provide greater accuracy, quicker responses and learning, and greater retention of information (Garris et al., 2002; Susi, Johannesson, \& Backlund, 2007b; Washburn, 2003).

\subsubsection{Difficulties of using games to study human behaviour}

Using serious games for research is often perceived negatively (Washburn, 2003). Because games have generally been designed for entertainment, applying games to research often results in the research being perceived as frivolous (Washburn, 2003). This presents a barrier to researchers using games as an effective tool for understanding behaviour (Washburn, 2003). While games can be used to study systems that would be unethical to study directly, experiments on human subjects (e.g. experimental games) can also be perceived as unethical (McCall \& Baillie, 2017; Redpath et al., 2018). These concerns and perceptions often stem from the early psychological simulation experiments that were conducted, such as the Stanford Prison Experiment of 1971 (Kozlov \& Johansen, 2010). Experiments such as these created a negative connotation with conducting experiments on human subjects, particularly gamification or simulation experiments.

Another challenge with using games to model human behaviour is iteration limitations (Redpath et al., 2018). Conceptual modelling methods can compute many 
iterations of many models relatively affordably and quickly. This is not always possible when using games to model human behaviour (Redpath et al., 2018). The equivalent process would involve running many repeats of many different game variations. Due to the time, financial and logistical restrictions, this is not possible. Therefore, acquiring large sample sizes can be more difficult when using applied methods as opposed to conceptual modelling methods.

Developing and using games to model human behaviour can also be expensive (Fletcher, 2017; Redpath et al., 2018). While complex conceptual modelling often requires powerful computers to run the algorithms, there are also costs associated with conducting applied research and games. Firstly, the equipment that is required to conduct the game must be purchased. This cost is highly dependent on the methodology used. Some games use financial rewards based on participant performance and an incentive to participate (Redpath et al., 2018). This research faced the financial challenge of providing rewards and incentives for participants. For one of the games, this totalled $\$ 250$ per game. Budgetary restrictions can also limit the ability to conduct large numbers of games and acquire large sample sizes.

Finally, there are uncertainties and difficulties associated with organising and researching people. Convincing people to give up their time to participate in a research project is difficult, despite financial incentives. There are also difficulties associated with convincing people to participate in research about criminal activity. It was not possible for me to use illegal hunters as participants due to ethical, health and safety and logistical limitations. Therefore, recreational hunting organisations were asked to provide participants as they were the next most contextually relevant population that was accessible. However, convincing recreational hunting organisations and their members to associate with research about illegal hunting proved difficult. Some did not want to be the subject of a study about illegal hunting.

\subsubsection{What makes a good game?}

When using experimental games as models for human behaviour, the games must meet certain requirements to ensure they will be effective. In designing the games, conceptual modelling techniques and experimental techniques were combined. As per Information Theoretics, models should be chosen or designed to be parsimonious (Bozdogan, 1987). This means that the model must achieve the desired level of explanation and accuracy while being as simple as possible (Bozdogan, 1987). Finding a balance between simplicity and accuracy 
is an important aspect of any model design, and a similar approach was used when designing and developing the games.

When designing a game as a model for human behaviour, the game should meet the objectives of the research while not being overly complex (Robinson, 2008). When models become overly complex it can become difficult to interpret the results (Robinson, 2008). By keeping the game simple, interpreting the results and understanding participant behaviour becomes easier. In this way, developing a game as a model can follow a similar process to designing an experiment whereby as many variables as possible are controlled for apart from one explanatory variable which is manipulated. This allows any observed difference to be associated to the explanatory variable, within a level of confidence. Simplicity in game design is also important for participants to understand the game. If the game is too complex, then participants may not understand the rules or their behavioural options, in which case understanding their behaviours and rationales becomes difficult.

Designing a game that meets the objectives of the study and represents the important aspects of the system being modelled is also important (Robinson, 2008). This can increase the researchers ability to draw robust conclusions about the system (Robinson, 2008). Deciding what aspects of the IWT system were important to include in the games was guided by an understanding of the system and how it operates. Adding components or complexity to a model is a method frequently used to increase the accuracy of the model and increase its ability to explain the system (Robinson, 2008).

\subsection{My game development process}

My research has developed experimental games to model human behaviour in ways that allow hypotheses to be developed and their predictions tested. These games are an operating model that represents and simplifies the IWT system. The games provide a method of studying and modelling human behaviour, testing the current theoretical understanding of how human behaviour responds to devaluation, and addressing the limitations associated with conceptual modelling techniques. To design and develop these games an integrative research approach has been used that combined modelling and experimental methodologies. The results of this research complement our theoretical understanding of devaluation strategies to further our understanding of the IWT system. 
In designing the games to be parsimonious, certain characteristics were required to ensure that they adequately represented the IWT system. The games had to involve participants hunting for items of value. A monetary reward was most suitable because the reward had to represent the rewards received by illegal hunters and be adjustable so that devaluation strategies could be applied with some rewards being of lesser value. The games also required a risk component to simulate the risk a hunter faces of being arrested or killed. To be consistent with the risk faced by illegal hunters, the risk component had to include a financial cost (representing a fine) and an opportunity cost (representing prison and confiscation of hunting equipment). Additionally, not only did there need to be a risk in locating a valuable item, but also an additional risk to claiming the item's value. Illegal hunters risk getting caught illegally entering a protected area. However, killing or harvesting an animal is likely to result in an additional risk because the probability of detection and severity of punishment would be expected to increase following the kill or harvest. Illegal hunters also risk getting caught after leaving the park while delivering the commodity to a buyer. Therefore, when a hunter locates a devalued animal, they must decide between:

1. Killing it and harvesting any remaining commodity, if any remains,

2. Killing it and not harvesting any remaining commodity,

3. Leaving it alive and continuing to search for a more valuable animal, and,

4. Leaving it alive and not continuing to search.

By introducing a risk in claiming an item, the participants are required to undertake the same decision-making process and determine whether claiming a devalued item is worth the additional risk.

Two games were developed. The first is referred to as the lucky-dip game, and the second is referred to as the scavenger-hunt game. These games began as a tool for teaching undergraduate students about complex socio-ecological-economic systems, like the IWT. These initial games were conducted by Associate Professor Wayne Linklater with the students of the 2016 and 2017 BIOL328: Conservation \& Behaviour Ecology undergraduate courses at Victoria University of Wellington. The games were followed by lectures on behavioural economics in a conservation context. I was involved with the design, running, improvement, and analysis of these games. Therefore, the two-years of using these games as a teaching tool were also used as pilot studies to improve and develop the games for this 
thesis. The objective of this process was to develop games for a more diverse public, and to develop games that were effective at testing theory and that could be rapidly replicated.

\subsubsection{Lucky-dip game pilot studies}

The lucky-dip games presented participants with a series of boxes that concealed different proportions of coloured balls. Yellow and red balls had monetary values (red balls had lower values as they represented devalued commodities), while blue balls represented risk (removal from the game and loss of all winnings). The boxes were arranged by increasing risk from right to left and increasing devaluation in the opposite direct from left to right. Participants were invited to select from the boxes of their choosing. They were told the proportion of balls in each box but could not see the ball they were selecting. Participants could choose not to play and could choose to stop playing at any time before selecting a blue ball and would then receive the value of their collected balls. This meant that studying how people respond to risk and reward was possible.

Two sets of pilot studies were conducted. The first was used as a tool to teach students about behavioural economics concepts in a conservation context. The second pilot study involved 10 acquaintances playing the lucky-dip games to determine game logistics for an application to the Victoria University of Wellington Human Ethics Committee to approve the study (Appendix A). This pilot study helped determine how long the games would take, how many participants and boxes would be required, the number of balls required per box, and the value of the balls to not exceed the budget.

In the first lucky-dip pilot study, the number of balls harvested increased as devaluation increased, but then dropped when devaluation reached 100\% (Figure 2.1.A). However, the number of balls 'killed' at $100 \%$ devaluation was similar to $70 \%$ devaluation, and larger than $0 \%$ and $30 \%$ devaluation (Figure 2.1.B). Therefore, this indicated that extensive devaluation may not provide any benefit over low levels of devaluation. The number of blue balls selected declined as the number blue balls in the boxes declined. 


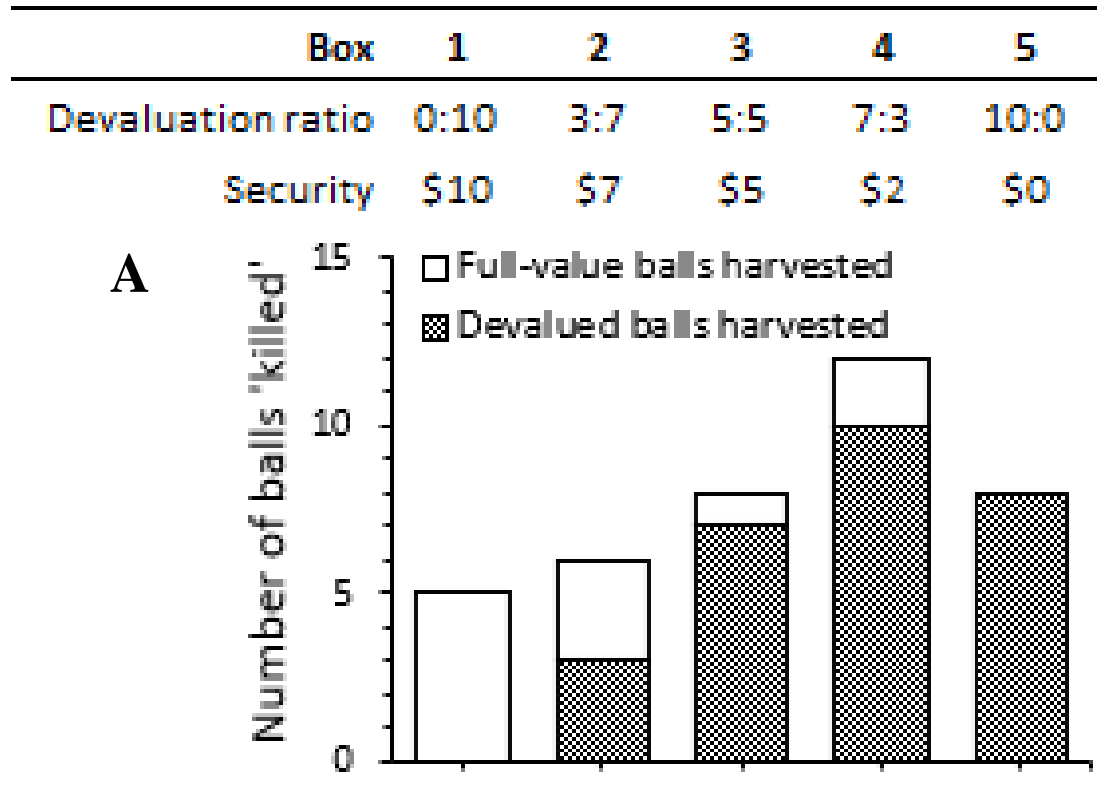

B

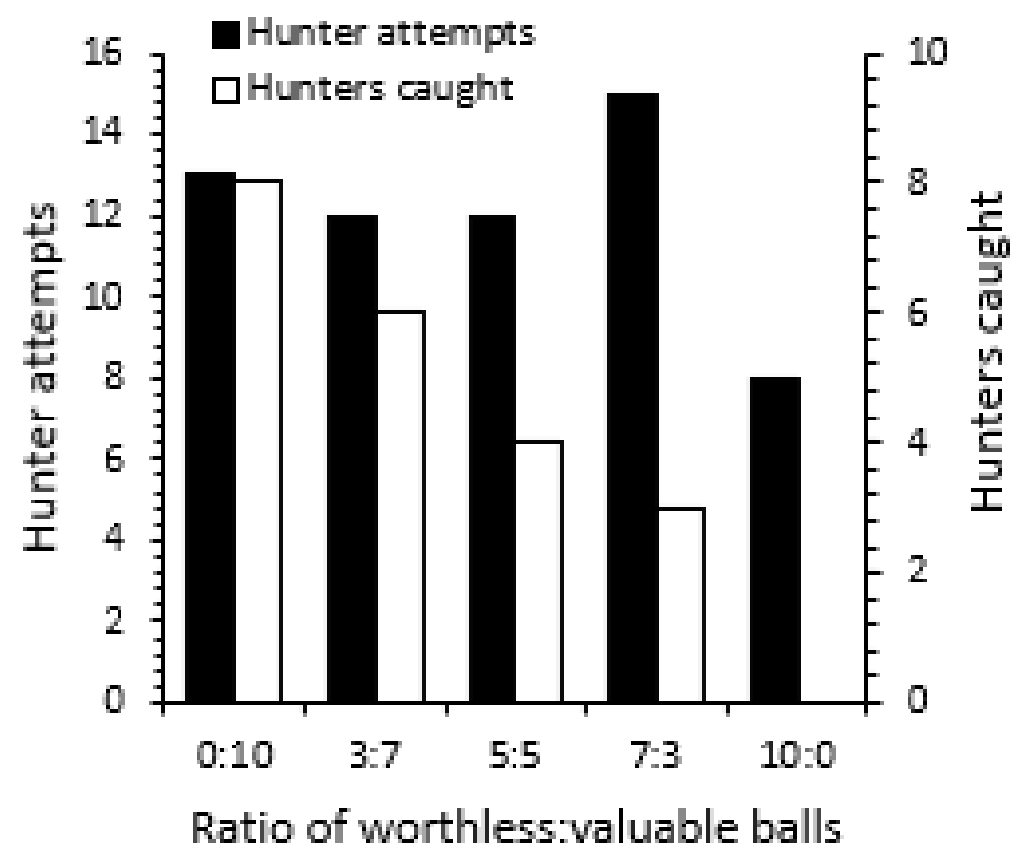

Figure 2.1. Results of the first lucky-dip pilot study showing (A) the number of balls 'killed', and (B) the number of hunter attempts and hunters caught in each game (Linklater \& Rudman, 2017). 


\subsubsection{Scavenger-hunt game pilot studies}

In the scavenger-hunt games, a group of participants had to search an outdoor area for hidden garden stakes worth money. Some of the items were worthless and represented devalued commodities. Guard participants were also recruited to protect the hidden items and catch hunters. Hunters that were visually identified by guards as either in possession of a stake or in the process of hunting for stakes were removed from the game. Guards were rewarded for every item the hunters did not 'kill'. Two sets of pilot studies were conducted on the grounds of the Victoria University of Wellington (VUW) Kelburn Campus. For each game, three volunteer guards and twenty randomly selected hunter participants were selected from the students enrolled in the course.

The 2016 pilot study suggested that intensive devaluation (between 50\% and 90\%) was successful at demotivating hunters and increasing stake survival. Participation, participant effort (Figure 5.2.B) and the number of stakes found (Figure 5.2.A) all dropped significantly when devaluation reached $90 \%$. However, participation and participant motivation spiked under $50 \%$ devaluation, suggesting that moderate levels of devaluation potentially incentivised harvesting.

The 2017 pilot study suggested that devaluation was unsuccessful at increasing stake survival (Figure 5.3.A). Many stakes were killed across all devaluation levels, and hunter motivation (participation and effort) was relatively constant across the first three games (Figure 5.3.B). Hunter motivation declined in Game 4. This was attributed to the students having an assignment due that week, and poor weather which discouraged participation. 


\begin{tabular}{rcccc}
\hline Game & $\mathbf{1}$ & $\mathbf{2}$ & $\mathbf{3}$ & $\mathbf{4}$ \\
\hline Stake ratio & $0: 20$ & $10: 10$ & $18: 2$ & $0: 20$ \\
Stake value & $\$ 5$ & $\$ 10$ & $\$ 50$ & $\$ 5$ \\
& & 凅 Worthless stakes "killed"
\end{tabular}
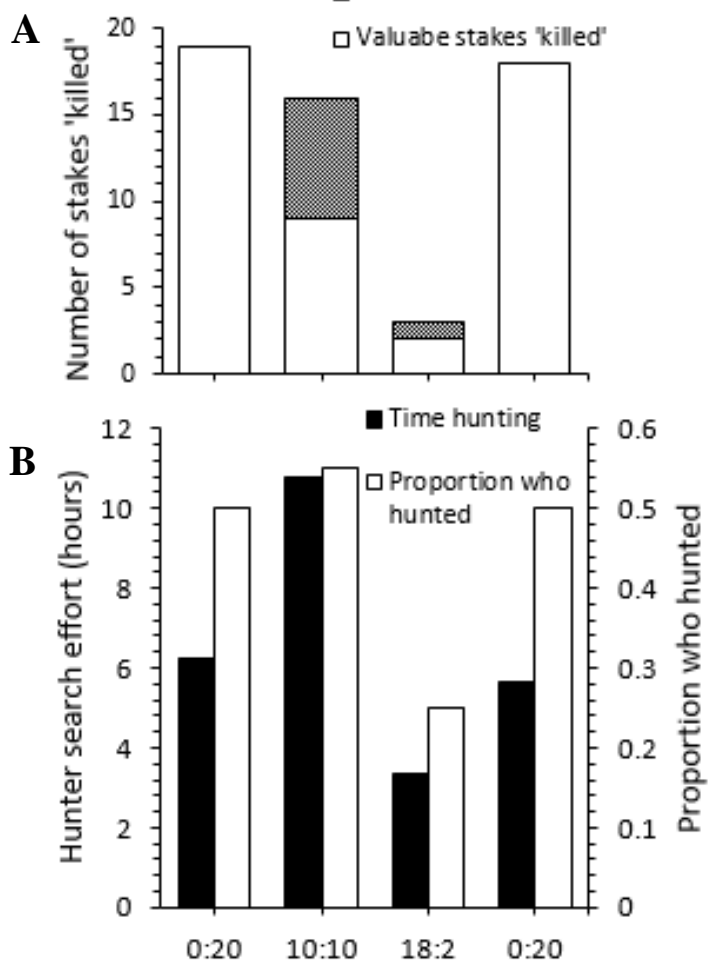

Ratio of worthless:valuable stakes

Figure 2.2. Results of the first

scavenger-hunt pilot study

(2016) showing (A) the number

of stakes 'killed', and (B) the

hunter search effort and

proportion of invited hunters that

participated in each game. All

variables show a decline in game

three (Linklater, Rudman, \&

Jackson, 2017).

\begin{tabular}{rcccc}
\hline Game & $\mathbf{1}$ & $\mathbf{2}$ & $\mathbf{3}$ & $\mathbf{4}$ \\
\hline Stake ratio & $0: 20$ & $10: 10$ & $18: 2$ & $0: 20$ \\
Stake value & $\$ 5$ & $\$ 10$ & $\$ 50$ & $\$ 5$ \\
& & WW Worthless stakes "killed
\end{tabular}

$\mathbf{A}=20 \ldots$ Valuabe stakes 'killed'
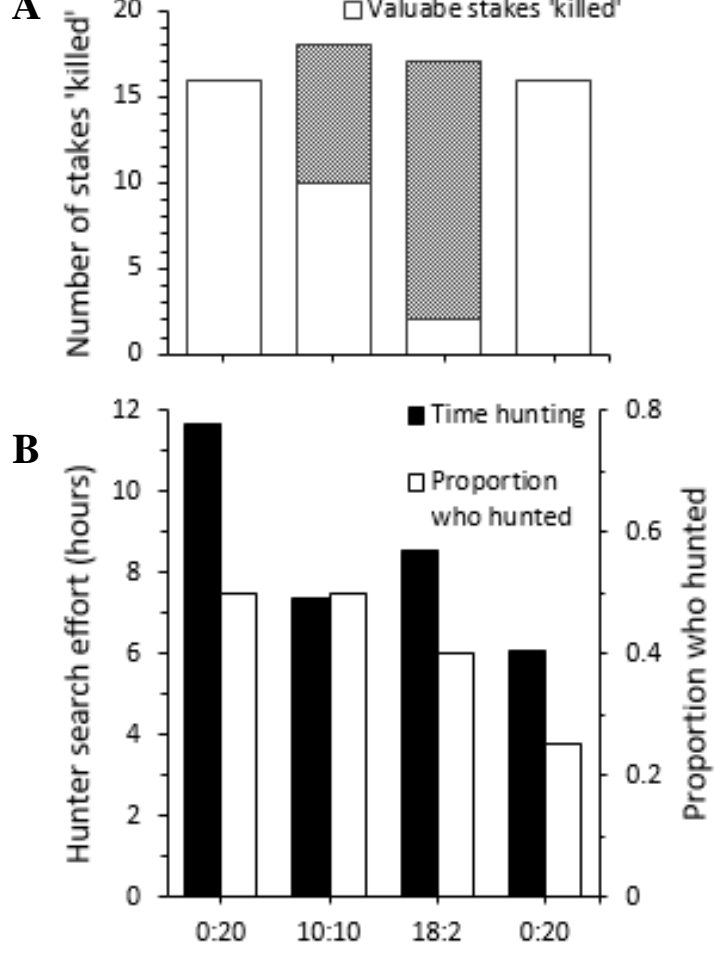

Ratio of worthless:valuable stakes

Figure 2.3. Results of the second scavenger-hunt pilot study (2017) showing (A) the number of stakes 'killed', and (B) the hunter search effort and proportion of invited hunters that participated in each game. No variable differs across all trials (Linklater et al., 2017). 


\subsubsection{Improvements made during the development process}

The lucky-dip game pilot studies differed from the final game applications in three main ways. Firstly, seven boxes were used rather than five as in the pilot studies. Using more boxes allowed for the inclusion of extreme levels of devaluation and risk (e.g. 100\% devaluation) which would be impossible to achieve in the real-world but are important to consider in an academic context. Secondly, the balls had lower values in the pilot studies with yellow balls worth $\$ 1$ and red balls worth 20 cents. In the final application yellow balls were increased from $\$ 1$ to $\$ 2$ to increase the reward potential, and three different red ball values (50 cents, 20 cents and worthless) were included to assess the importance of the magnitude of devaluation (proportion of the commodities value that is lost following devaluation). Thirdly, the pilot study games were played by three participants at a time, with participants replaced when they withdrew or selected a blue ball. In the final application, ten participants played simultaneously so that the number of participants exceeded the number of boxes. This forced participants to decide whether to queue at boxes or move to boxes that are less ideal. The need to video record these games (with participant permission) was also apparent because with ten participants and seven boxes, it would be impossible to record all participant's selections and behaviours in real-time. The recordings were reviewed for data collection and moderation purposes.

Table 2.1. The number and distribution of yellow, red and blue balls in each of the five boxes in the lucky-dip pilot study games.

\begin{tabular}{llll}
\hline Box & Yellow & Red & Blue \\
\hline 1 & 10 & 0 & 10 \\
2 & 7 & 3 & 7 \\
3 & 5 & 5 & 5 \\
4 & 3 & 7 & 2 \\
5 & 0 & 10 & 0 \\
\hline \hline
\end{tabular}


To make the scavenger-hunt games more representative of the IWT system, improvements were made between the 2016 and 2017 pilot studies, and before conducting the final game applications. Firstly, the total value of each pilot study game was $\$ 100$. This was raised to $\$ 250$ to increase the reward potential. Participants of the 2016 pilot study were told the context of the research prior to participating. However, some students reported associating the stakes with rhino. Their conservation bias influenced their decision making, primarily on whether to kill a worthless stake. Therefore, in the 2017 pilot study and this thesis, participants were only told the context of the research after participating.

During the pilot studies the games were conducted over multiple days between 8am and $5 \mathrm{pm}$ with the 'surviving' stakes relocated before 8am the following morning. This relocation of stakes represented the unpredictable movement of animals in the wild and provided an incentive for a hunter to kill a worthless stake to prevent finding it again on a subsequent day. The 2016 pilot study took place over two days involving one overnight relocation of stakes. The 2017 pilot study took place over three days involving two overnight relocations of stakes. Making the games longer was necessary as the number of worthless stakes killed in the first pilot study was not consistent with evidence of hunter responses to real-life devaluation strategies. From the post-game surveys that were conducted, $80 \%$ of participants expressed that they felt no incentive to' kill' a devalued stake. However, it is known that hunters will kill a devalued animal to prevent tracking and finding it again in the future (Milner-Gulland, Leader-Williams, \& Beddington, 1994). This alteration may have been the reason why more worthless stakes were laid down in the 2017 pilot study. For the final game applications, the games were shortened to 2.5 hours to ensure that members of the public would be able to participate. It might be expected, therefore, that participants will be less likely to 'kill' a worthless stake because less worthless stakes were 'killed' in the shorter of the two pilot studies.

Finally, for guards the value of the stakes had to be consistent even though the values changed for hunters. In the 2016 pilot study, each stake had the same value for both guards and hunters. Guards were therefore only incentivised to protect stakes that had value because they were not rewarded for any surviving worthless stakes. This is not realistic. All rhino (devalued or not) are valued equally by guards and will receive equal levels of protection. Therefore, even though from a hunter's perspective some stakes were worthless, in the 2017 pilot study all stakes were worth an equal \$5 (\$100 in total) to guards across all four games. This change may have also contributed to more worthless stakes being laid down in the 2017 
pilot study. Participants had more incentive to lay down devalued stakes as this would ensure that the guards were not rewarded for the stake.

\subsubsection{The benefits of conducting two games}

Conducting both types of game (lucky-dip and scavenger-hunt) was important to the success of the project as the games complement each other. In the lucky-dip games, each box represented a park. Therefore, examining how the action of one park might impact hunting in surrounding parks was possible. The value of devalued red balls could also be manipulated between games to determine whether the level or magnitude of devaluation results in different human behavioural outcomes. Finally, conducting many lucky-dip games was possible because they were simple, quick and affordable. The scavenger-hunt games were more complex, longer and more expensive than the lucky-dip games. The scavenger-hunt games gave participants more opportunity to exhibit behavioural nuances, cooperative deals and complex strategies which were less likely in the lucky-dip game. Additionally, the scavenger-hunt games involved human guards which created competition between hunters and guards, allowed for retributive behaviours, and for cooperative strategies to develop between hunters and guards.

Conducting only one of these games would have been detrimental to the project and limited its scope. The lucky-dip games were unable to thoroughly examine complex human behaviours that are elicited by the scavenger-hunt games and are vital to understanding the complexity of the IWT system. If only the scavenger-hunt games had been conducted the project would have been data limited because these games were longer, more expensive and required much more organisation. Therefore, conducting many repeats and variations of these games would not have been possible. Conducting both games was important to the success of the project because the games complement each other, and the limitations of each game are addressed by the other 



\section{Methods}

\subsection{Lucky-dip games}

The lucky-dip games were conducted at five public events (e.g. carnivals, galas and fairs) across the Wellington Region. Potential participants were given an information sheet (Appendix B) and were required to sign a consent form (Appendix C) to play. Participants were not told the context of the research before participating but were provided with a brochure after the game explaining the context (Appendix D). Signs were set up and pamphlets handed out to explain and advertise the game. The games were recorded by two GoPro cameras so that the footage could be viewed for data collection and validation purposes. Seven 54 litre black storage boxes with lids concealed different proportions of yellow, red and blue balls and participants were told the proportion of balls in each box at the start of the game. Figure 3.1 shows how the stall was set up at each event. The lids locked in place and had a $10 \mathrm{~cm}$ diameter hole cut in them. This hole had a section of foam matting glued over it with a cross cut in it. Therefore, participants could reach through the hole and foam to select a ball, without being able to see the ball being selected. Each lid also had a 2litre white container secured to it for participants to discard unwanted balls in to. Ten participants continuously selected balls from any of the storage boxes, but only one participant could select from each box at a time. Thus, queues to select from certain boxes formed. Each participant wore a unique identifying code around their neck so that they could be differentiated in the video footage, and was given a bucket labelled with the same code to collect balls in. Yellow balls represented full-value commodities worth $\$ 2$, and red balls represented devalued commodities worth either 50 cents, 20 cents or zero cents (worthless). Blue balls represented risk, and selecting a blue ball resulted in the participant being removed from the game, and having their accumulated balls confiscated. When a blue ball was selected, it was returned to the box that it came from.

Participants could choose to leave the game at any time before selecting a blue ball. They would then receive the value of their collected yellow and red balls in cash. The game ended when all participants had either selected a blue ball or decided to withdraw and claim their winnings. Three types of game were conducted. Two of these included red balls. Of these, three variations of each were conducted with the three red-ball values. Therefore, a total of seven game variations were conducted. 


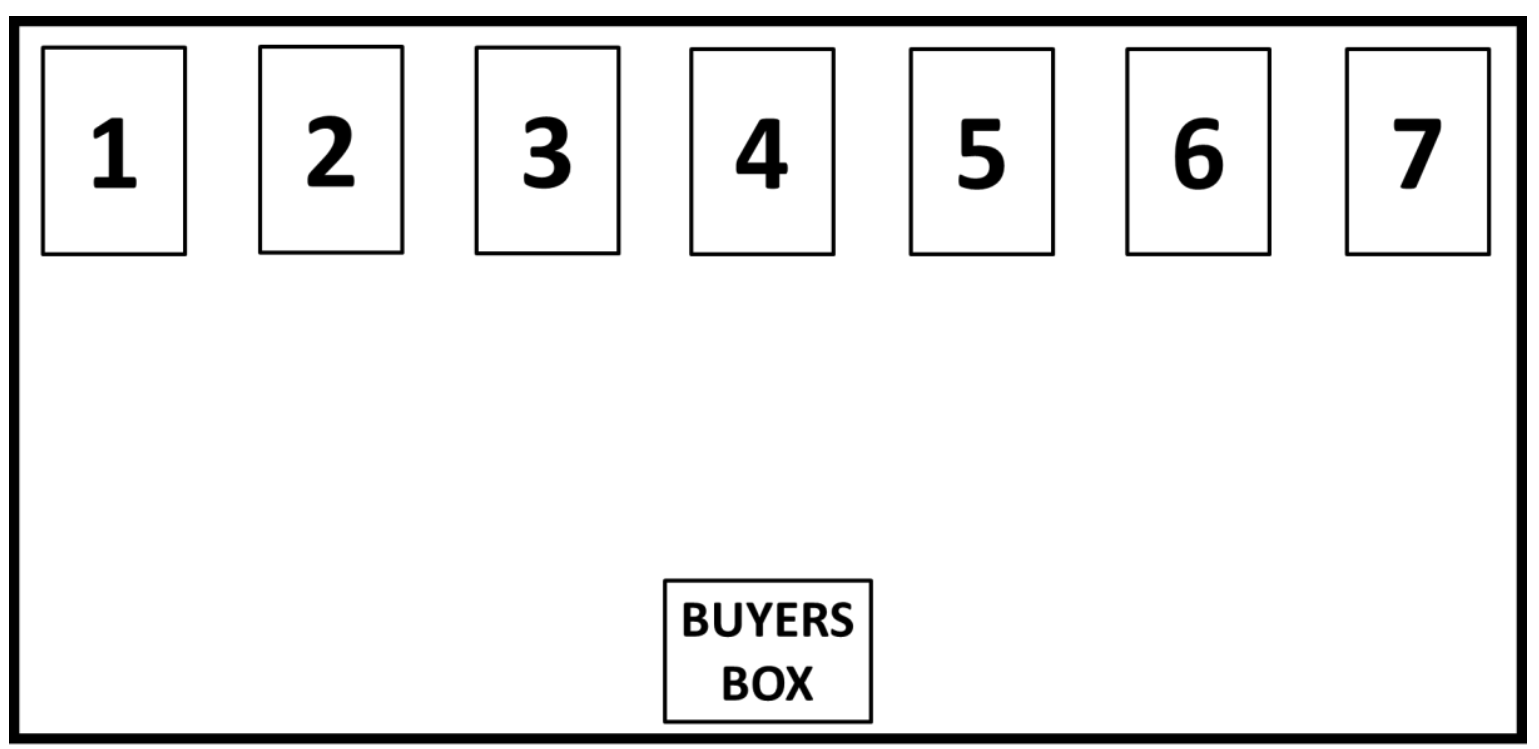

Figure 3.1. A diagram of how the stall was set up at the public events. The seven storage boxes are represented at the top, while the box at the bottom represents the buyer's box. Participants were located between the seven front boxes and the buyer's box. 
To claim the value of each selected yellow or red ball, participants had to select a ball from an eighth box called the 'buyer's box' containing one blue and nine pink balls (Figure 3.1). Selecting the blue ball from the buyer's box would again result in the participant being removed from the game, and having their accumulated balls confiscated. Selecting a pink ball allowed the participant to select again from one of the initial seven boxes. All balls selected from the buyer's box (blue or pink) were returned to the box. The risk at the buyer's box represented the risk of selling the commodity to a buyer and had to be completed every time a yellow or red ball was claimed. Participants were instructed to take one of three actions when they selected a ball.

1. Keep it, and therefore select a ball from the buyer's box to claim its value,

2. Return it to the box that it came from and select a new ball (from any box) or,

3. Discard it into the white container on top of each box and select a new ball (from any box).

Figure 3.2 presents a decision tree for a participant's potential actions. Returning a devalued red ball to its original box meant that the ball would remain in the game and could be selected again. This represented finding a devalued animal and deciding not to kill or harvest it. Discarding a devalued red ball into the ice-cream container meant that the ball was removed from the game and could not be selected again. Therefore, any value it held was lost. This represented finding a devalued animal and deciding to kill it, but not harvest its commodity. Because these actions represented not harvesting the animal's commodity, there would be no risk associated with selling the commodity to a buyer. Therefore, the participant could select another ball without having to select a ball from the buyer's box. 


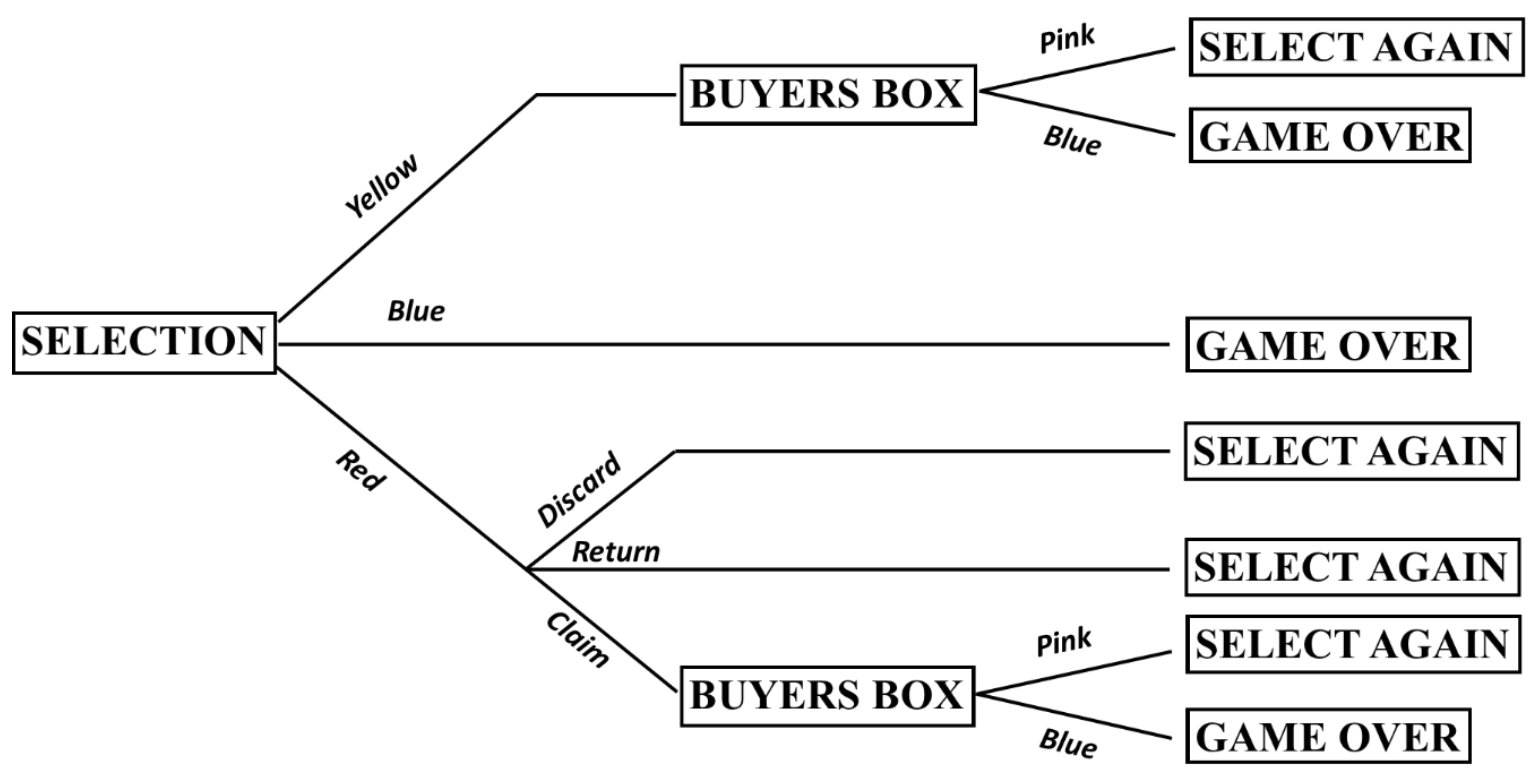

Figure 3.2. Decision tree showing a participant's possible actions upon selecting a ball depending on which coloured ball is selected, and the outcomes of each action. 


\subsubsection{Variable red ball values}

The value of red balls was changed between games to investigate how the magnitude of devaluation affected participant behaviour. A concern with current devaluation strategies applied to rhino is that some valuable horn remains after devaluation. Additionally, horns regrow meaning that the valuable horn replenishes after devaluation. Therefore, the effectiveness of current devaluation strategies is questioned because they do not render the commodity or animal completely worthless. By manipulating the value of the red balls across games, the influence that the magnitude of devaluation (red ball value relative to yellow ball value) had on participant behaviour could be assessed. Across games, red balls were worth either 50 cents, 20 cents or zero cents (i.e., worthless).

\subsubsection{Game one: Security game}

The security games included only yellow and blue balls. Each of the seven boxes contained ten yellow balls while the number of blue balls (risk) decreased from ten in Box 1 to zero in the Box 7 (Table 3.1). The boxes were arranged in an order of decreasing risk (decreasing blue balls) from left to right. This game investigated how different levels of risk alone influences participant behaviour and the outcome of the game.

\subsubsection{Game two: Devaluation game}

The devaluation games included only yellow and red balls. Each of the seven boxes contained ten balls (Table 3.1). Box 1 contained ten yellow balls which represented no devaluation of a population. As devaluation increased along the boxes, yellow balls were replaced by devalued red balls. Box 7 contained only ten red balls representing the $100 \%$ devaluation of a population. The boxes were arranged in an order of increasing devaluation (increasing ratio of red-yellow balls) from left to right. Because this game included red balls, three variations were conducted with red balls worth 50 cents, 20 cents and zero cents. This game investigated how different levels of devaluation alone influenced participant behaviour and the outcome of the game. 
Table 3.1. The number and distribution of yellow and blue balls at the start of each (a) security game, and (b) devaluation game.

(a)

\begin{tabular}{ccc}
\hline \hline Box & Yellow & Blue \\
\hline 1 & 10 & 10 \\
2 & 10 & 9 \\
3 & 10 & 7 \\
4 & 10 & 5 \\
5 & 10 & 3 \\
6 & 10 & 1 \\
7 & 10 & 0 \\
\hline \hline
\end{tabular}

(b)

\begin{tabular}{ccc}
\hline \hline Box & Yellow & Red \\
\hline 1 & 10 & 0 \\
2 & 9 & 1 \\
3 & 7 & 3 \\
4 & 5 & 5 \\
5 & 3 & 7 \\
6 & 1 & 9 \\
7 & 0 & 10 \\
\hline
\end{tabular}




\subsubsection{Hypothesis model}

From the simple security and devaluation games, distributions were formed of how different levels of risk (security game) and devaluation (devaluation game) influenced the survival of the commodity. An empirical hypothesis (model) was created to predict the outcome of a trade-off game that includes both blue balls (security) and red balls (devaluation). This was done by summing the average survival of balls at each box in the security and devaluation games. The resulting hypothesis model therefore predicted the expected survival of balls at each box in a trade-off game that includes both security (blue balls) and devaluation (red balls). This model has risk increasing from left to right on the $x$-axis, and devaluation increasing in the opposite direction from right to left (Figure 3.3). This model was used to test for an interaction between the motivators of risk and reward for participants. If there is no interaction between motivators of risk and rewards then the average survival of balls at each box in the trade-off games should be statistically similar to the hypothesis model (our null hypothesis). 


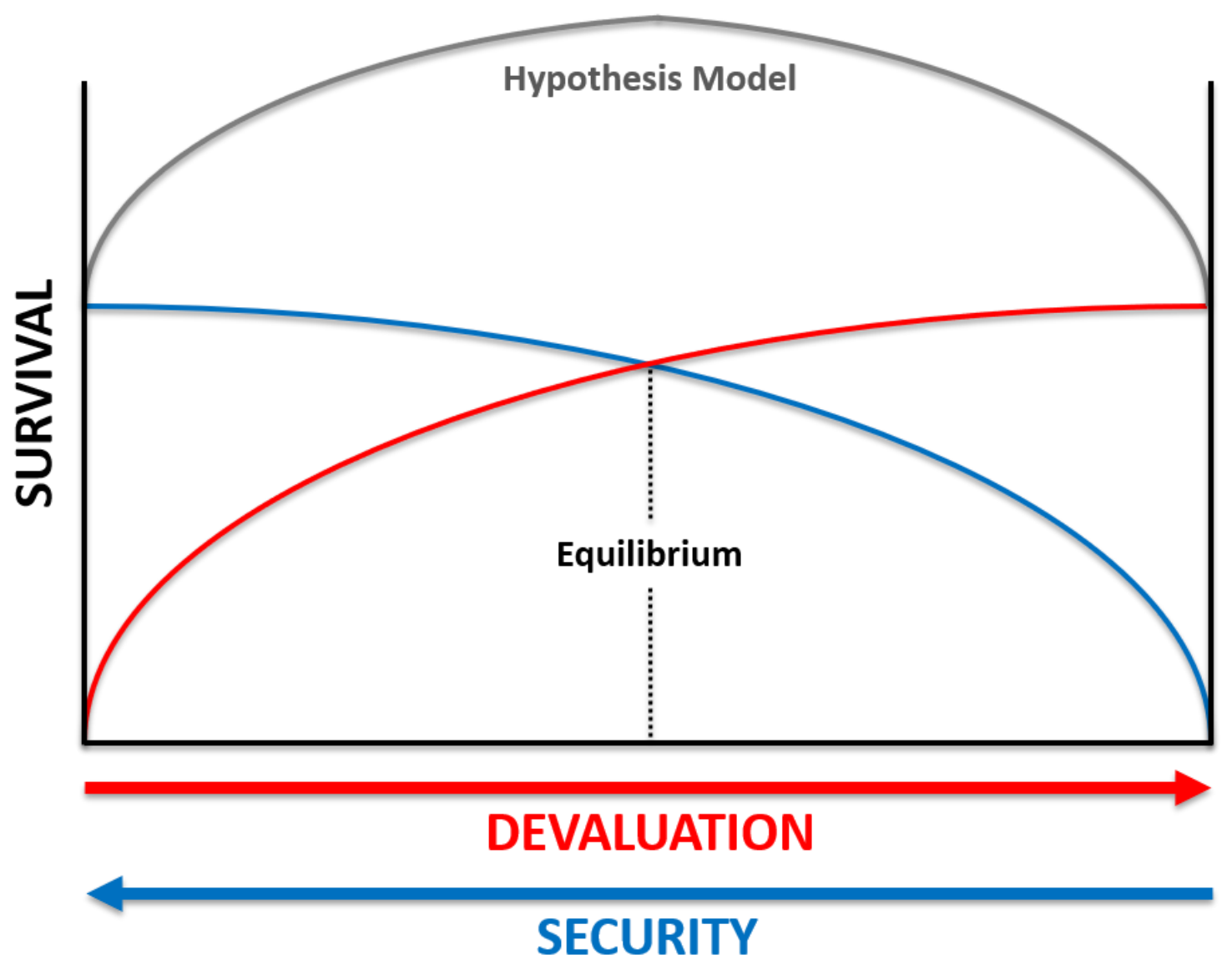

Figure 3.3. An example of what the hypothesis model for predicting commodity survival may look like. It combines a security (risk) distribution (blue) and devaluation distribution (red). Where these two separate models meet is the point where values of risk and reward deliver similar outcomes in terms of commodity survival. The grey line represents the hypothesis model which predicts the expected survival of balls at each box in a trade-off game that includes both blue balls and red balls. This is created by summing the average survival of balls at each box in the security and devaluation games. 


\subsubsection{Game three: Trade-off game}

The trade-off games included yellow, red and blue balls. Each of the seven boxes contained ten yellow and/or red balls (Table 3.2). Box 1 contained ten yellow and ten blue balls, with no red balls. This box represented the highest reward potential, but also the highest risk potential. As devaluation occurred, yellow balls were replaced with devalued red balls, and simultaneously the number of blue balls equally decreased. The boxes were arranged in an order of increasing devaluation (increasing ratio of red-yellow balls) from left to right and increasing risk (blue balls) in the opposite direction from right to left. This resulted in the Box 7 containing just ten red balls. Again, this inverse relationship represented a 1:1 financial trade-off between security and devaluation. Because this game included red balls, three variations were conducted with red balls worth 50 cents, 20 cents and worthless. This game investigated how inversely changing levels of both risk and devaluation influences participant behaviour and how the game is played. Comparisons could therefore be made between the results of this game and the hypothesis model to determine whether commodity survival conformed to the empirical hypothesis model (null hypothesis). ${ }^{1}$

\footnotetext{
${ }^{1}$ When referring to the lucky-dip game throughout this thesis, the following terminologies are used. When referring to the devaluation games the devaluation percentage is used to refer to boxes. For example, “50\% devaluation” refers to Box 4 with 5 red balls and 5 yellow balls. When referring to the security games the number of blue balls is used to refer to boxes, because the number of yellow balls was constant at 10 and only the number of blue balls changed. For example, "3 blue balls" refers to Box 5. Finally, when referring to the trade-off games, the ratio of blue balls (risk) to red balls (devaluation) is used to refer to boxes. For example, "Box 7:3" refers to Box 3 with 7 blue balls and 3 red balls. These notations are used so the reader does not have to memorise the number of balls of each colour in each box.
} 
Table 3.2. The number and distribution of yellow, red and blue balls at the start of each lucky-dip trade-off game.

\begin{tabular}{cccc}
\hline Box & Yellow & Red & Blue \\
\hline 1 & 10 & 0 & 10 \\
2 & 9 & 1 & 9 \\
3 & 7 & 3 & 7 \\
4 & 5 & 5 & 5 \\
5 & 3 & 7 & 3 \\
6 & 1 & 9 & 1 \\
7 & 0 & 10 & 0 \\
\hline \hline
\end{tabular}




\subsubsection{Dynamic games}

Having learned from the devaluation, security and trade-off games, a more dynamic version of the lucky-dip games was developed and tested. In these dynamic games, additional participants were included as guards at each box (population of balls). Before the start of play, the guard at each box was instructed to set their desired proportion of red, yellow and/or blue balls in their box. Importantly, they were given the following trade-off constraints.

1. the number of yellow plus red balls must equal 10. That is, the number of yellow balls in each box was 10 minus the number of red balls.

2. the number of blue balls needed to be in a 1:1 inverse relationship with red balls. That is, the number of blue balls in each box was also 10 minus the number red balls, and therefore equalled the number of yellow balls.

Thus, before starting the game guards established the amount of risk and devaluation for the population of balls as a trade-off between devaluation and security strategies. Guards were told that they would be paid $\$ 1$ for every yellow and red ball remaining in their box at the end of the game. Guards were paid a constant value for each surviving ball, rather than the actual value of the ball. This is because in the real-world a devalued animal (e.g. rhino) is as valuable to the guards as an animal that has not been devalued. Because these games were more dynamic and complex than the lucky-dip games with more interactions between participants, the number of boxes was reduced from seven to three. Limiting the number of boxes, and therefore the number of guards, made data collection and analysis manageable. The number of hunters remained at ten. The trade-off games did not last long, and if the number of hunters was reduced, the games would have been too short to receive sufficient data.

The hunting and guard participants of these games were undergraduate students from the 2018 BIOL 328 (Behaviour and Conservation Ecology) class at Victoria University of Wellington (VUW), and postgraduate students in the School of Biological Sciences at VUW. A student population was used because these games were also a treated as a pilot study. These games had not been conducted previously, and therefore a convenient sample was used. Additionally, this game was developed late into this thesis so there was not time to take it to a more general population as with the other lucky-dip games. Eight games were conducted with the same population of fifteen participants. In four games red balls were 
worth 20 cents, and in the other four games red balls were worth 50 cents. The ten hunters and three guards were randomly selected from the pool of fifteen participants before each game.

The hunters were shown the composition of balls in each box before the game started. Once started, hunters could select balls from any box of their choosing. For every yellow or red ball kept, a ball had to be selected from the buyer's box. Red balls could also be returned or discarded. Selecting a blue ball resulted in the participant being removed from the game and having their accumulated balls and their associated winnings confiscated. Hunters could decide to stop playing at any point before selecting a blue ball and were then paid the value of balls in their bucket.

After the beginning of play, guards could call a temporary stop to the game (timeout) to change the composition of balls in their box by applying the same constraints as when the composition of each box was established. If a yellow ball was added a red ball was removed, and a blue ball added. If a red ball was added, a yellow and a blue ball were removed. If a blue ball was added, a red ball was removed and a yellow ball added. Based on the results from the previous games of how hunters behaved, a hypothesis was formed predicting how the guards would behave. This hypothesis could be tested in the dynamic game.

In total 34 lucky-dip and 8 dynamic games were completed. The data from one of the lucky-dip games was not used because it was realised retrospectively that the pre-game instructions given to participants were incorrect. Therefore, data from 33 games were analysed (Table 3.3). Eleven of these were devaluation games with red balls worth 50 cents in five games, 20 cents in three games, and worthless in three games. Four security games were conducted (without red balls). Eighteen trade-off games were conducted with red balls worth 50 cents in ten games, 20 cents in three games, and worthless in five games. Only four security games were conducted because the results of these games were consistent with low variance. More repeats of the devaluation and trade-off games were conducted where variation in outcomes was greater. 
Table 3.3. A summary of the number of games of each variation completed.

\begin{tabular}{lcccc}
\hline \hline & \multicolumn{3}{c}{ Red Ball Value } & \\
\cline { 2 - 4 } Game & $\mathbf{5 0}$ cents & $\mathbf{2 0}$ cents & Worthless & Total \\
\hline Devaluation & 5 & 3 & 3 & $\mathbf{1 1}$ \\
Security & N/A & N/A & N/A & $\mathbf{4}$ \\
Trade-off & 10 & 3 & 5 & $\mathbf{1 8}$ \\
& & & Total & $\mathbf{3 3}$ \\
Dynamic & 4 & 4 & 0 & 8 \\
& & & Trand Total & $\mathbf{4 1}$ \\
\hline \hline
\end{tabular}




\subsubsection{Data collection}

The following data was recorded:

- the number and colour of balls that each participant collected,

- the number and colour of balls remaining in each box at the end of the game,

- whether participants claimed, returned or discarded each red ball selected,

- the number of blue balls selected from each box, and

- where hunters were selecting balls from or queueing.

This data was collected by photographing the balls in each participants' bucket with the unique identifying code visible, and by photographing the balls remaining in each storage box and ice-cream container at the end of each game. Data on the number of red balls returned to their box, where hunters were selecting balls from and where they were queueing was gathered from the video recordings. The video recordings were also analysed to validate the photographic data collection. Participants were asked to complete a post-game survey to gather self-reports of their behaviour and experience (Appendix E). Each participants' unique identifying code was recorded on their survey in case there was a need to correspond their answers with the game results. While the data collected from the game was informative of the participants' behaviours, the surveys provided important information on their rationales for these behaviours. 


\subsection{Scavenger-hunt games}

Organisations were contacted to ask whether they would be willing to participate in the research. They were provided with an information sheet (Appendix F) and were asked to sign a consent form to participate (Appendix G). Between 8 and 20 participants were recruited for each game from consenting organisations. Initially, organisations were asked to provide 20 participants. However, all organisations expressed difficulty recruiting this number and this requirement was therefore reduced to between 8 and 20 participants. Due to difficulties recruiting hunting organisations, the scope was expanded to include any outdoor organisation (e.g. tramping/hiking clubs, orienteering clubs; Table 3.4). Participants were provided with an information sheet (Appendix $\mathrm{H}$ ) and were asked to sign a consent form if they were willing to participate (Appendix I). Again, participants were informed of the context of the research after the game. These games were conducted at Wellington City Council (WCC) and Greater Wellington Regional Council (GWRC) parks. The parks used were WCC Karori Park, GWRC Belmont Regional Park and GWRC Pakuratahi Forest. While it would have been preferable to conduct all the games at the same location (Karori Park), this was not practical for some organisations that were located further afield. Therefore, to encourage participation, two games were conducted at locations more convenient for the participating organisation (Figure 3.4). When this occurred, locations were chosen that were similar to Karori Park in size, track network, vegetation and terrain to provide consistency and allow us to pool responses and compare results between locations. 
(A)

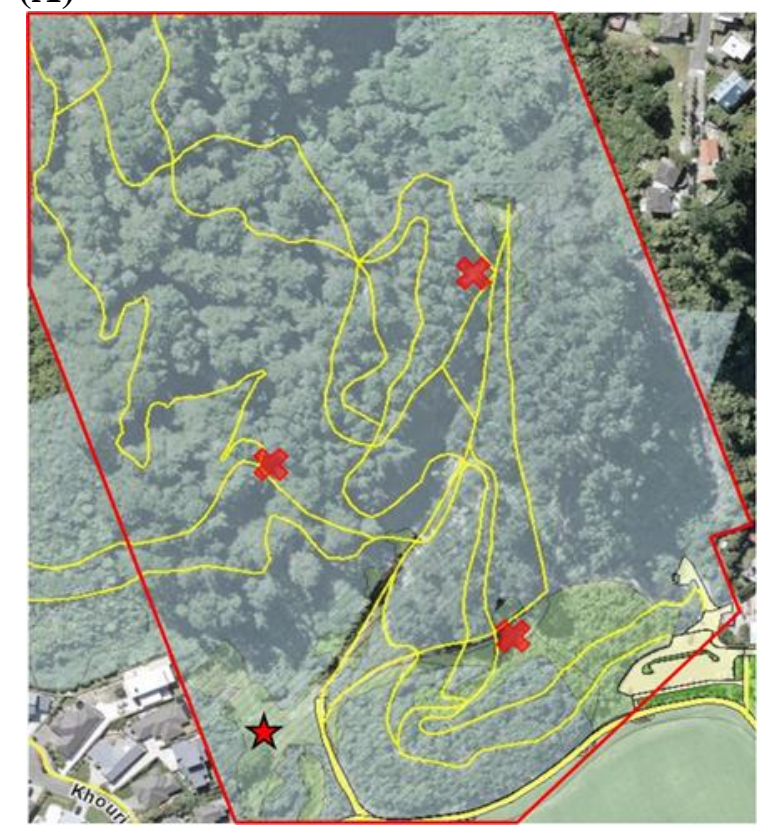

(C)

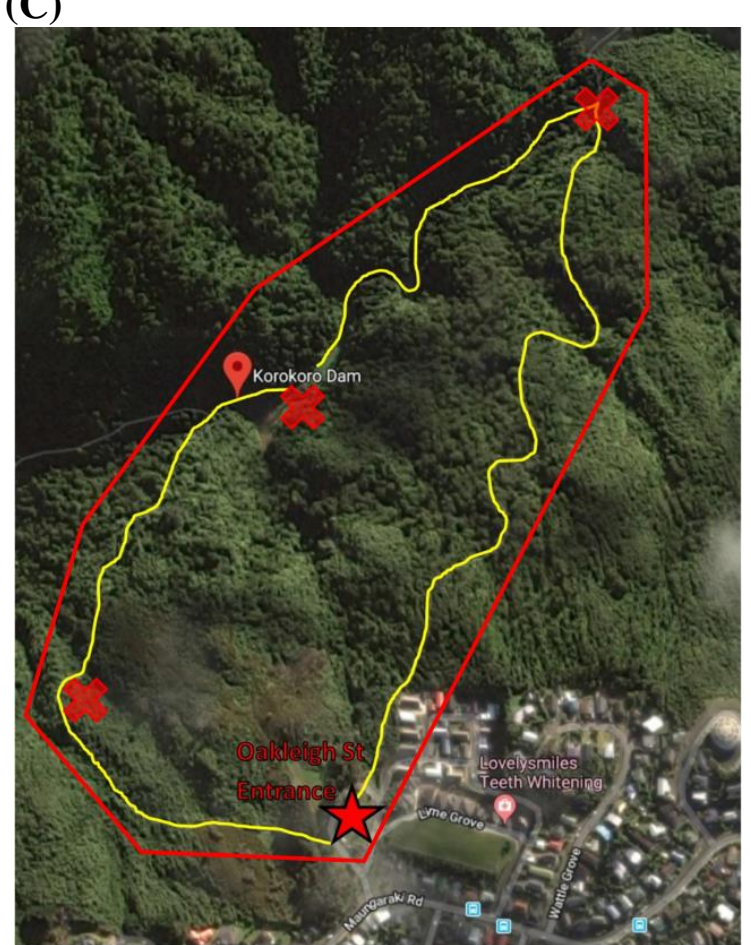

(B)

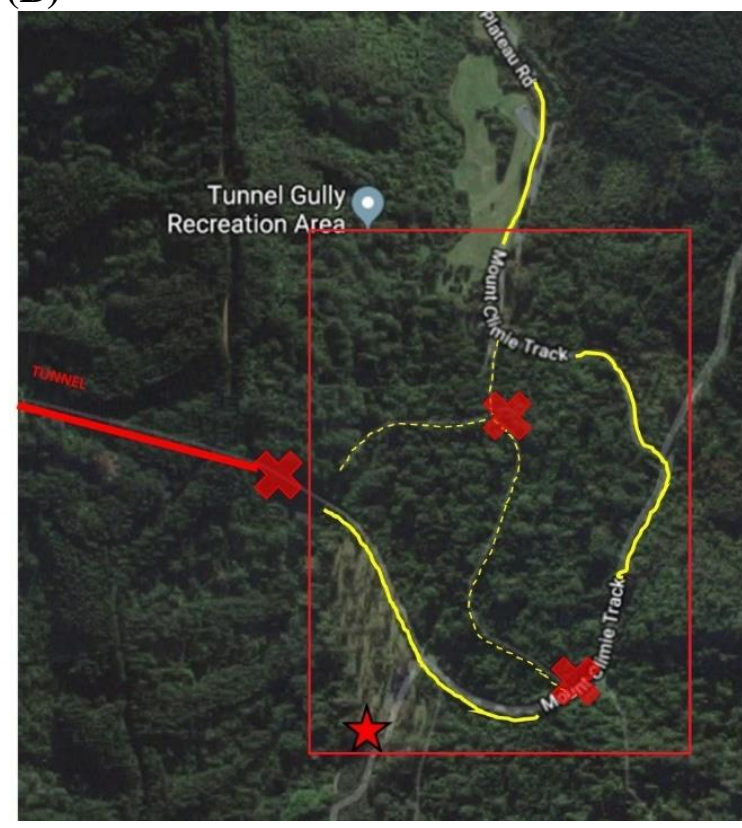

Figure 3.4. Maps of the three sites where scavenger-hunt games were conducted.

A) Karori Park, B) Pakuratahi Forest, and C) Belmont Regional Park. The red line indicates the game boundary and the yellow line indicates the track network along which the stakes were situated. The red stars indicate the assembly point, and the red crosses indicate where delivery boxes were placed for participants to deliver stakes to. Maps retrieved from Google Maps (Imagery: Google 2018, Map Data: Google 2018). 
Table 3.4. A summary of the type of organisation each game was conducted with, where each game was conducted, the number of participants and the devaluation percentage.

\begin{tabular}{ccccc}
\hline \hline Game & Organisation Type & Site & Participant \# & Devaluation \% \\
\hline 1 & Orienteering Club & Karori Park & 14 & $0 \%$ \\
2 & Hunting Club & Pakuratahi Forest & 8 & $50 \%$ \\
3 & Tramping Club & Belmont Regional Park & 18 & $90 \%$ \\
4 & Tramping Club & Karori park & 8 & $50 \%$ \\
\hline \hline
\end{tabular}


In each game, $20 \times 1.2 \mathrm{~m}$ high, $10 \mathrm{~mm}$ diameter garden stakes were hidden in wooded sections of the chosen site. Each stake was painted a unique colour and was assigned a monetary value. The stakes were distributed along a track network. They were placed off the track but were visible from the track. Between 8 and 20 hunter participants operated in pairs to attempt to locate the 20 stakes. Each hunting pair wore matching coloured t-shirts. Two external guard participants were also recruited and were instructed to operate as a pair to protect the stakes. The guards were also tasked with locating and reporting the identity of hunters in possession of a stake to the authorities (the game coordinator). For a hunter pair to be caught, the guard pair had to report their t-shirt colour and the colour of the stake in their possession. This would result in the hunter pair being excluded from playing the remainder of the game, and all the stakes they had collected and delivered being confiscated without reward. To claim the value of a found stake, hunters had to deliver it to the buyer (also the game coordinator) at the assembly point, or place the stakes in one of four bins that were placed along the track network (Figure 3.4).

Hunters were instructed to take one of three actions when they found a stake. Either:

1. Retrieve the stake ('kill' it) and deliver it to claim the financial reward,

2. Remove the stake from the ground ('kill' it) but leave it laying where it was found (no reward claimed), or,

3. Leave the stake standing ('alive').

Each game consisted of three half-hour stages. The end of play in each stage was signalled with an air horn, which meant all participants had to return to the assembly point until play was restarted. During these breaks, any 'killed' stakes were removed from the game and any 'surviving' stakes were relocated to simulate the unpredictable movement of a living animal commodity. Hunters were instructed to keep a record of the stakes that they located and what they did with them.

Four games were conducted with different organisations. In each game, $\$ 250$ could be earned by hunters delivering the stakes they found to the buyer. The total value earned was donated to the participating organisation. Across multiple trials, different numbers of stakes were devalued. Stakes that were devalued and worth nothing could be identified by a rubber band placed around the stake just above where the stake entered the ground. Therefore, participants were required to closely inspect the stakes to determine their status. In the first 
game, all 20 stakes were worth an equal $\$ 12.50$ each (\$250/20 stakes). This was the control game to determine how participants behaved when no devaluation is applied. In the three remaining games a proportion of the stakes were marked as worthless. In Game 2, ten stakes were marked worthless (50\% devaluation), with the ten remaining stakes worth $\$ 25$ each (\$250/10). In Game 3, 18 stakes were marked worthless (90\% devaluation), with the remaining two stakes worth $\$ 125$ each (\$250/2). In Game 4, the 50\% devaluation game was repeated. A fifth game was planned to repeat the $90 \%$ devaluation game, but an organisation that was willing and able to participate could not be found. The guards received $\$ 12.50$ (\$250/20) for every stake that was 'surviving' at the end of each game. Again, guards were paid a constant value for each stake (valuable or devalued) because a devalued animal is worth as much to guards as an animal that has not been devalued. Participants could elect to withdraw from the game at any time to safeguard the value of any stakes they had already collected and delivered.

\subsubsection{Data collection}

The data collected from these games included the number of valuable and devalued stakes surviving and killed at the end of the game and each stage. Of particular interest was what participants did with each devalued stake that they found. Participants were provided with timesheets and were asked to record the colour of any stakes found and what they did with them. This allowed data to be collected on any behavioural nuances that were exhibited. For example, some participants retrieved valuable stakes, but not deliver them immediately. Instead they would hide them so they could be recollected later and could not be relocated between stages. This allowed them to wait and deliver them at a safer time. Each participant was provided with a pedometer and step readings were taken at the end of each game stage. This data was used as a proxy for motivation (Menickellia \& Hastie, 2014) based on the assumption that a more motivated participant will travel more distance and therefore take more steps over the half hour stage. If participants decided to withdraw from the game prior to its completion, this was recorded and also used as proxy for motivation. Participants were again asked to complete a post-game survey which provided the rationales for their behaviours (Appendix J). Participants were asked to score their motivation for each of the three devaluation levels on a Likert scale. 


\subsection{Online survey}

A survey was developed and distributed to recreational hunters through hunting organisations. It presented respondents with legal hunting scenarios that economically simulated the scenarios faced by illegal hunters (Appendix K). The survey was created through the online software Qualtrics. The committees of hunting organisations were contacted and asked if they would be interested in participating in the research. Drafts of the survey were distributed to the committees so that committee members could provide feedback and recommendations for the completion of the survey. The organisations that agreed to participate were then sent a link to the online survey for them to distribute to their membership. The survey was active for a month.

For context, the scenario provided to respondents was the hunting of white-tailed deer for venison on Stewart Island, New Zealand. The survey comprised of two main Sections (1 and 2), with Section 1 divided in to two sub-Sections (A and B). Section 1A was comprised of six questions which were presented to respondents in a random order. Section 1B was comprised of one question and was always presented to respondents immediately following Section 1A. Section 2 was comprised of four questions which were also presented to respondents in a random order.

Section 1A presented respondents with a venison hunt scenario across four fictional hunting parks. In the survey, the term "block" was used instead of "park" as this is the terminology used in the hunting community. In this thesis the term "park" is used. Each park presented a different proportion of male deer of different age class (Figure 3.5). Each age class was assigned a monetary value so that the total value of venison in each park was a constant $\$ 4,000$. Therefore, the only difference was the distribution of the $\$ 4,000$, and not the total value of each hunting opportunity. Park 1 presented twenty stags valued at \$200 each; Park 2 presented ten stags worth $\$ 100$ and ten stags worth $\$ 300$ each; Park 3 presented fifteen stags worth \$100 and five stags worth \$500 each; and Park 4 presented eighteen stags worth $\$ 100$ and two stags worth $\$ 1,100$ each. Each respondent was presented with all paired combinations of parks, totalling six questions, and was asked which park they would prefer to hunt in.

Section 1B presented a ranking question. The same four parks were used as in Section 1A (Figure 3.5), and respondents were asked to rank the four parks from one being their most preferred park, to four being their least preferred park. 


\section{$\underline{\text { Park } 1}$}

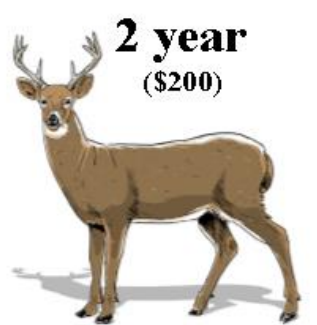

20

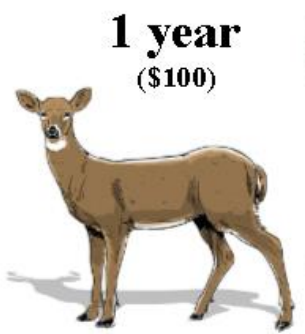

10

\section{$\underline{\text { Park } 2}$}

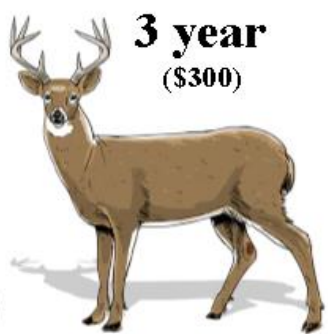

10

\section{$\underline{\text { Park } 3}$}

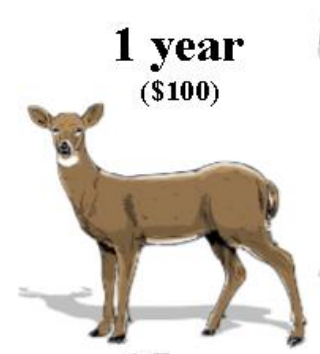

15

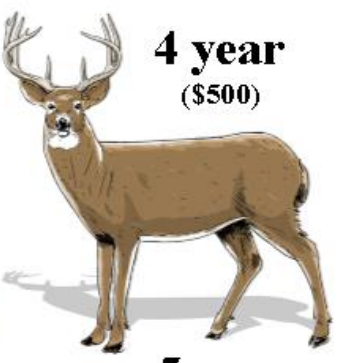

5

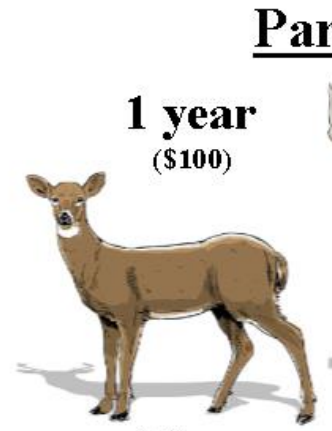

18 $\underline{\text { Park } 4}$

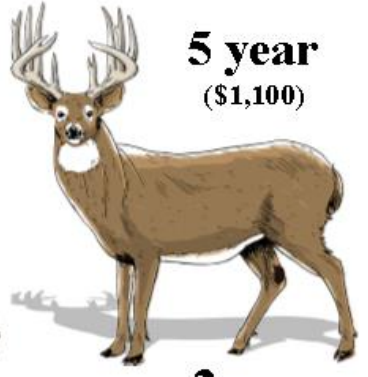

2

Figure 3.5. The four parks that were presented to respondents in Section 1 of the survey including the park number, the age off the deer the corresponding value, plus the number of deer of each age in the park. Images retrieved from Honeycutt and Orndorff (2016). 
In Section 2 of the survey, respondents were presented with a three-day balloted trophy (antler) hunt scenario. Four questions were presented, each representing a hunting park (Figure 3.6). Each of the four parks contained five deer that could be hunted. A proportion of the five deer in each park were yearlings with no trophy (worthless) while the remaining stags were mature with large trophies. Park 1 presented four yearlings and one mature stag, Park 2 presented three yearlings and two mature stags, Park 3 presented two yearlings and three mature stags, and Park 4 presented one yearling and four mature stags.

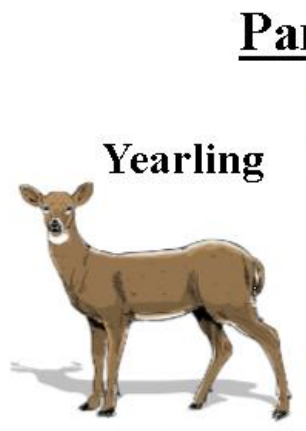

4 $\underline{\text { Park } 1}$

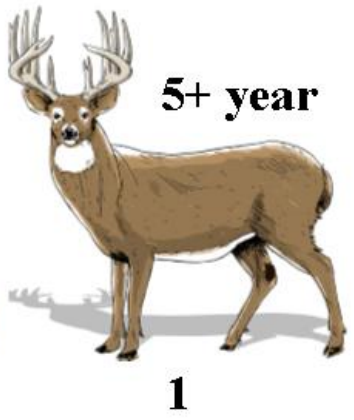

Park 3

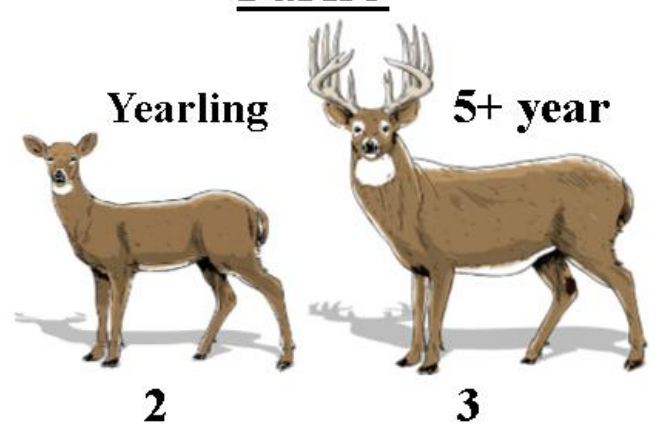

$\underline{\text { Park } 2}$

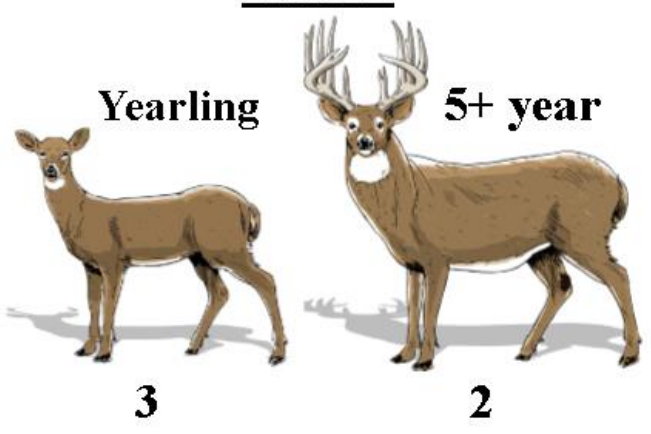

$\underline{\operatorname{Park} 4}$

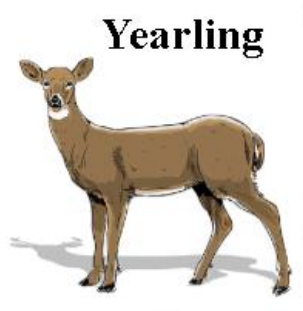

1
$5+$ year

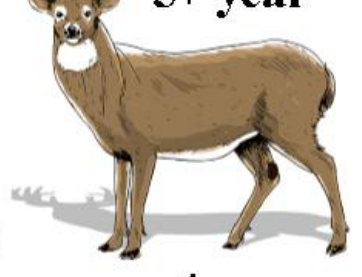

4

Figure 3.6. The four parks that were presented to respondents in Section 2 of the survey including the park number, the age off the deer and the number of deer of each age in the park. Images retrieved from Honeycutt and Orndorff (2016). 
The ballot occurred in two stages. Firstly, the hunters had to select from the hunting ballot to receive a hunting slot. There were 20 hunters and 16 hunting slots, meaning the hunter had an $80 \%$ chance of hunting that day. The selected respondents received a hunting slot, meaning they then selected from the deer ballot to determine which of the five deer they would hunt. Respondents were told that a random number generator was used to select which of the deer they could hunt. In fact, the survey was designed so that the respondents were always assigned a yearling for all four questions. This is because yearlings represented devalued animals, and how hunters responded to devalued animals was of particular interest. Respondents were then asked to pick between two actions. Firstly, they could either return the yearling they had selected to the ballot. This meant they could immediately select a new deer to hunt the following day, but also meant that they risked selecting the same yearling again. Alternatively, they could discard the yearling they selected from the ballot. This meant that they did not risk selecting the same yearling again. However, before selecting the deer they would hunt the following day, they had to re-enter the hunting ballot, giving them a $20 \%$ chance of being unable to hunt the next day. This section of the survey was designed so that for each question the probability of selecting a mature stag with a second selection was the same, regardless of whether the respondent chose to return or discard the yearling (Table 3.5). It was therefore hypothesised that there would be no difference in the number of people who selected return and discard for each question. 
Table 3.5. Assuming the respondent selected a worthless yearling with their first selection, this table shows the chance that the participant would receive a stag on their next selection if they returned or discarded the yearling. Having returned the yearling to the ballot, the chance of getting a stag on the next selection is based on the number of stags and yearlings in the ballot. Having discarded the yearling from the ballot, the chance of getting a stag on the next selection is based on the penalty of only having an $80 \%(4 / 5)$ chance of selecting again, multiplied by the chance of getting a stag with one less yearling in the ballot. The table shows that for all four questions, the chance of getting a stag is no different depending on whether the yearling is returned or discarded from the ballot.

\begin{tabular}{ccccc}
\hline \hline & & \multicolumn{2}{c}{ Chance of getting a stag on second } & \\
\cline { 3 - 3 } Question & Ratio (stag : yearling) & Return & Discard & Best option \\
\hline 1 & $4: 1$ & $4 / 5$ & $4 / 5 \times 1=4 / 5$ & No difference \\
2 & $3: 2$ & $3 / 5$ & $4 / 5 \times 3 / 4=3 / 5$ & No difference \\
3 & $2: 3$ & $2 / 5$ & $4 / 5 \times 1 / 2=2 / 5$ & No difference \\
4 & $1: 4$ & $1 / 5$ & $4 / 5 \times 1 / 4=1 / 5$ & No difference \\
\hline \hline
\end{tabular}




\subsection{Statistical analysis}

Statistical analyses were conducted using R 3.5.1 software with significance assessed at the $\alpha$ $=0.05$ significance level $(\mathrm{R}$ Core Team, 2017). ANOVA tests were used to test for variation in outcomes between boxes in the lucky-dip games. When the data did not fit the assumptions of an ANOVA, a Kruskal-Wallis test was used. As much of the data was count data, it frequently failed to meet the assumption of normality of errors. Data transformations for these count datasets were not possible due to the datasets being zero-inflated. Mann-Whitney tests were used for post-hoc analysis of the Kruskal-Wallis tests. The raw pedometer data did not meet the assumptions of an ANOVA, but a log transformation of the data was possible and was used to meet the assumption of normality of errors. For these ANOVAs, Tukey tests were used for the post-hoc analyses.

A model of expected survival in trade-off games was created by summing the average survival of balls in the security and devaluation games for each box. This model was used to form hypotheses about the outcome of the trade-off games. The expected survival from the hypothetical model was plotted alongside the observed survival from the trade-off games. A 95\% confidence interval was plotted around the observed average survival of balls in the trade-off games to determine whether there was a significant difference to the hypothesis model. If the corresponding data point on the hypothesis model fell within this $95 \%$ confidence interval, it was concluded that there was no significant difference between the models.

Two pairs of chi-square tests were conducted for the lucky-dip games. Standardised residuals with a Bonferroni correction were used for post-hoc analyses. The first pair of tests were conducted to test the independence between red ball outcome (kept, returned or discarded) against red ball value $(\$ 0, \$ 0.20$ and $\$ 0.50)$, and red ball outcome against game type (devaluation and trade-off). Both tests were significant, so logistic mixed-effects models were conducted using the glmer function within the lme4 package in $\mathrm{R}$ (Bates et al., 2015). These models were used to determine whether there was an interaction between red ball value and game type on the outcome of red balls. Logistic models were used due to difficulties with running multinomial mixed-effect models. The first logistic mixed-effects model asked whether each red ball selected was kept? (yes $=0 ;$ no $=1$ ). The second model asked, if a red ball was not kept, was it returned or discarded? (return =0; discard =1). Red ball value and game type were treated as fixed effects with an interaction term, while game and participant 
were treated as random effects, with participant nested within game. Before testing fixedeffect models, I tested whether and which random-effects were important to model power by adopting an Information Theoretic approach. Universal models were compared with all fixedeffects that varied in all possible combinations of random effects (full model with both random effects nested, just the game random effect; just the participant random effect; and no random effects) (Zuur et al., 2009). An AIC using the maximum likelihood (ML) method of estimation was suitable because of the large sample size $(\mathrm{N}>300$, parameters $\geq 6$; Burnham $\&$ Anderson, 2002). The universal model with participant nested within game was used as it was the substantially supported model.

A second pair of chi-square tests were conducted to test the independence between red ball outcome against site, and red ball outcome against game type. Both tests were significant, so logistic mixed-effects models using the glmer function within the lme4 package in $\mathrm{R}$ were again used to determine whether there was an interaction between red ball value and game type on the outcome of red balls (Bates et al., 2015). The first logistic mixedeffects model asked whether each red ball selected was kept? (yes =0; no =1). The second model asked, if a red ball was not kept, was it returned or discarded? (return =0; discard = 1). Site and game type were treated as fixed effects with an interaction term, while game and participant were treated as random effects, with participant nested within game. Again, universal models with all possible combinations of random effects were compared using AIC under the ML method of estimation (Burnham \& Anderson, 2002; Zuur et al., 2009). The universal model with the participant nested game random effect was used as it was again the substantially supported model.

Participant surveys from the trade-off lucky-dip games were divided by those who won money (winners) and those who did not because they selected a blue ball (losers). This was done to determine whether those who won played the game differently from those who lost, and to determine whether there was a consistent strategy among those who won. When possible, chi-square tests were conducted to test for a difference in the answers provided by winning participants and losing participants. When the data did not meet the assumptions of a chi-square test due to small sample sizes, a Fishers exact test was used.

We received 333 responses to the online survey. Of these, 173 (52\%) were completed responses, and 160 (48\%) were partially completed responses. Because the survey sections, and questions within sections, were presented to respondents in a random order, all questions 
received between 159 and 208 responses. However, because a partially completed response rate of $48 \%$ is high, chi-square tests were conducted for every question comparing the full data set, the data set of complete responses and the data set of partially complete responses. None of these chi-square analyses indicated a statistically significant difference for any of the questions across the data sets (most significant association: $\chi^{2}{ }_{2}=5.0, \mathrm{~N}=412, P=0.084$ ). Therefore, it was acceptable to use the full data set including partially completed surveys for my analyses.

To analyse the pairwise comparison results from Section 1A of the online survey, a Bradley-Terry model was applied to the data using the BradleyTerry 2 package in $\mathrm{R}$ (Turner \& Firth, 2012). The Bradley-Terry model is a probability model that predicts paired comparison outcomes (Turner \& Firth, 2012). For Section 2 of the online survey, the first two questions that presented a higher proportion of yearlings were combined for analysis, as were the second two questions that presented a higher proportion of stags. This produced a $2 \times 2$ contingency table for which a chi-square test was conducted. 



\section{Results}

\subsection{Lucky-dip games}

\subsubsection{The devaluation and security games}

The survival of balls varied between boxes in the devaluation and security games in the hypothesised directions (Figure 4.1). Ball survival increased with increasing ratios of devaluation and security (Devaluation: Kruskal-Wallis, $\mathrm{H}(d f=6)=26.2, P<0.001$; Security: $\mathrm{H}(d f=6)=24.2, P<0.001$; Figure 4.1). Post-hoc tests reveal that $100 \%$ devaluation significantly improved survival compared to all other devaluation rates. The survival of balls when $70 \%$ and $90 \%$ were devalued was also significantly improved compared to boxes with no devalued balls (Table 4.1). The survival of balls at boxes with 1 and 3 blue balls was statistically lower than boxes with 5, 7,9 and 10 blue balls. The survival of balls at boxes with no blue balls was lower than all other boxes except the box with 1 blue ball. 


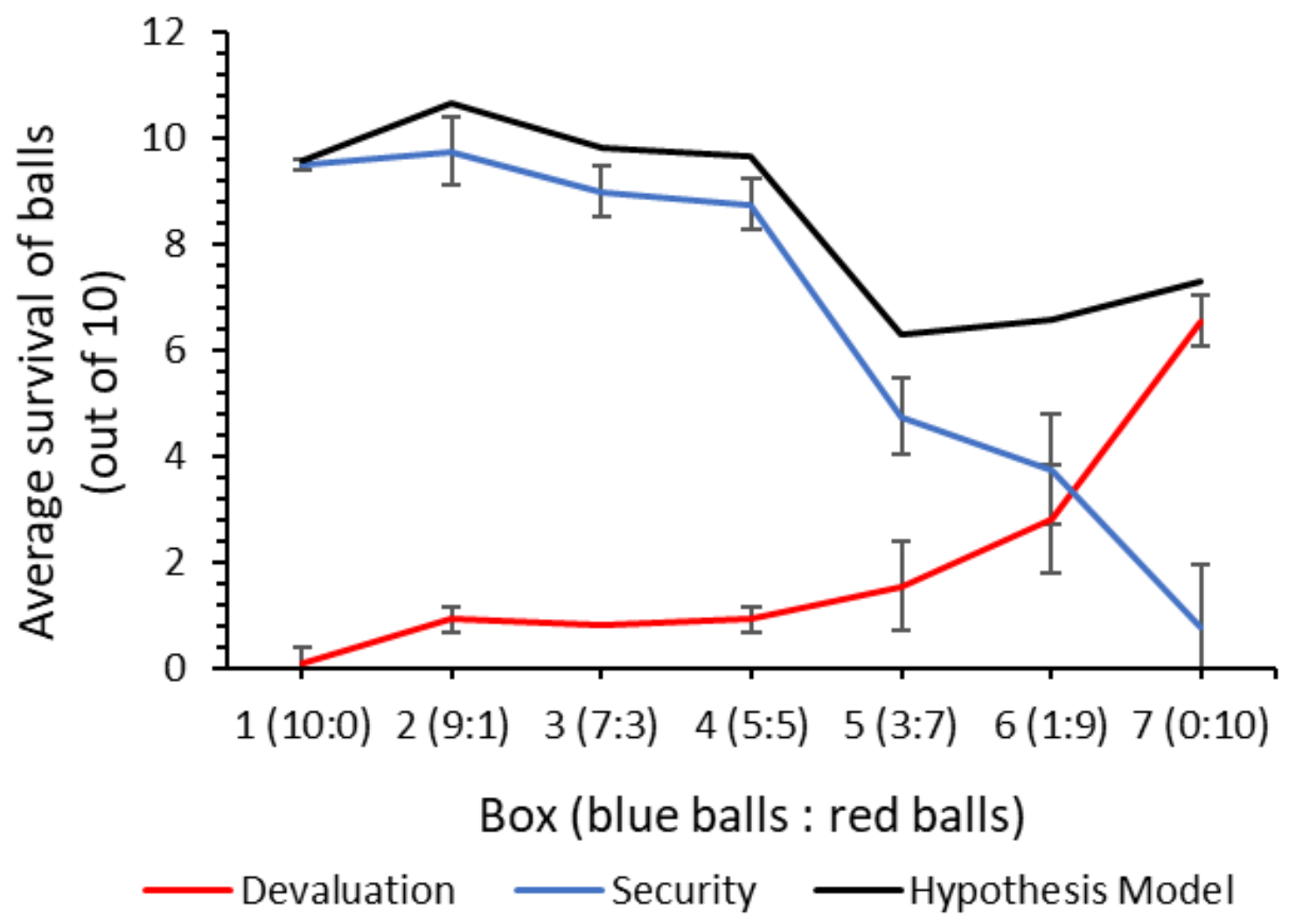

Figure 4.1. The average survival of balls (out of 10) in the devaluation (red) and security (blue) lucky-dip games. Devaluation in the form of red balls increases from left to right, and risk in the form of blue balls increases in the opposite direction from right to left. The black line represents the hypothesis model of expected survival in trade-off games by summing the average survival of balls in the security and devaluation games for each box. Error bars are \pm 1 standard error. 
Table 4.1. Mann-Whitney post-hoc analysis $p$-values for the pairwise comparisons of total survival at each pair of boxes in (A) devaluation games, and (B) security games. Bold face indicates values that are significant at the 0.05 significance level.

\begin{tabular}{c|cccccc}
\multicolumn{1}{l}{ (A) } \\
\hline \hline Devaluation (\%) & $0 \%$ & $10 \%$ & $30 \%$ & $50 \%$ & $70 \%$ & $90 \%$ \\
\hline $10 \%$ & 0.262 & - & - & - & - & - \\
$30 \%$ & 0.261 & 1.00 & - & - & - & - \\
$50 \%$ & 0.241 & 0.933 & 1.00 & - & - & - \\
$70 \%$ & $\mathbf{0 . 0 2 1}$ & 0.237 & 0.301 & 0.417 & - & - \\
$90 \%$ & $\mathbf{0 . 0 4 4}$ & 0.268 & 0.170 & 0.170 & 0.725 & - \\
$100 \%$ & $<\mathbf{0 . 0 0 1}$ & $\mathbf{0 . 0 0 1}$ & $\mathbf{0 . 0 0 1}$ & $\mathbf{0 . 0 0 1}$ & $\mathbf{0 . 0 0 6}$ & $\mathbf{0 . 0 1 7}$ \\
\hline \hline
\end{tabular}

(B)

\begin{tabular}{c|cccccc}
\hline \hline Security (blue balls) & 0 & 1 & 3 & 5 & 7 & 9 \\
\hline 1 & 0.078 & - & - & - & - & - \\
3 & $\mathbf{0 . 0 2 9}$ & 0.657 & - & - & - & - \\
5 & $\mathbf{0 . 0 2 6}$ & $\mathbf{0 . 0 2 6}$ & $\mathbf{0 . 0 2 7}$ & - & - & - \\
7 & $\mathbf{0 . 0 2 0}$ & $\mathbf{0 . 0 2 0}$ & $\mathbf{0 . 0 2 1}$ & 0.453 & - & - \\
9 & $\mathbf{0 . 0 2 6}$ & $\mathbf{0 . 0 2 6}$ & $\mathbf{0 . 0 2 7}$ & $\mathbf{0 . 0 5 8}$ & 0.060 & - \\
10 & $\mathbf{0 . 0 2 7}$ & $\mathbf{0 . 0 2 7}$ & $\mathbf{0 . 0 2 8}$ & 0.134 & 0.181 & 0.608 \\
\hline \hline
\end{tabular}




\subsubsection{The trade-off games}

In the trade-off games commodity survival differed significantly between some of the boxes at the devaluation and security extremes (Kruskal-Wallis, $\mathrm{H}(d f=6)=14.4, P=0.025$;

Figure 4.2). Post-hoc tests reveal that ball survival at Box 10:0 (10 blue balls and 0 red balls) was significantly higher than Boxes 5:5 to 1:9, and ball survival at Box 9:1 was higher than Boxes 7:3 to 1:9 (Table 4.2).

Average ball survival at each box in security and devaluation games were added to create the hypothesis model of expected survival at each box in the trade-off games (the black line in both Figure 4.1 and Figure 4.2). For boxes 10:0 to 5:5, observed average ball survival was worse than the hypothesis model. Additionally, at Box 3:7 average ball survival was higher than expected from the hypothesis model. Only at Boxes 1:9 and 0:10 was average ball survival not different from the hypothesis model. 


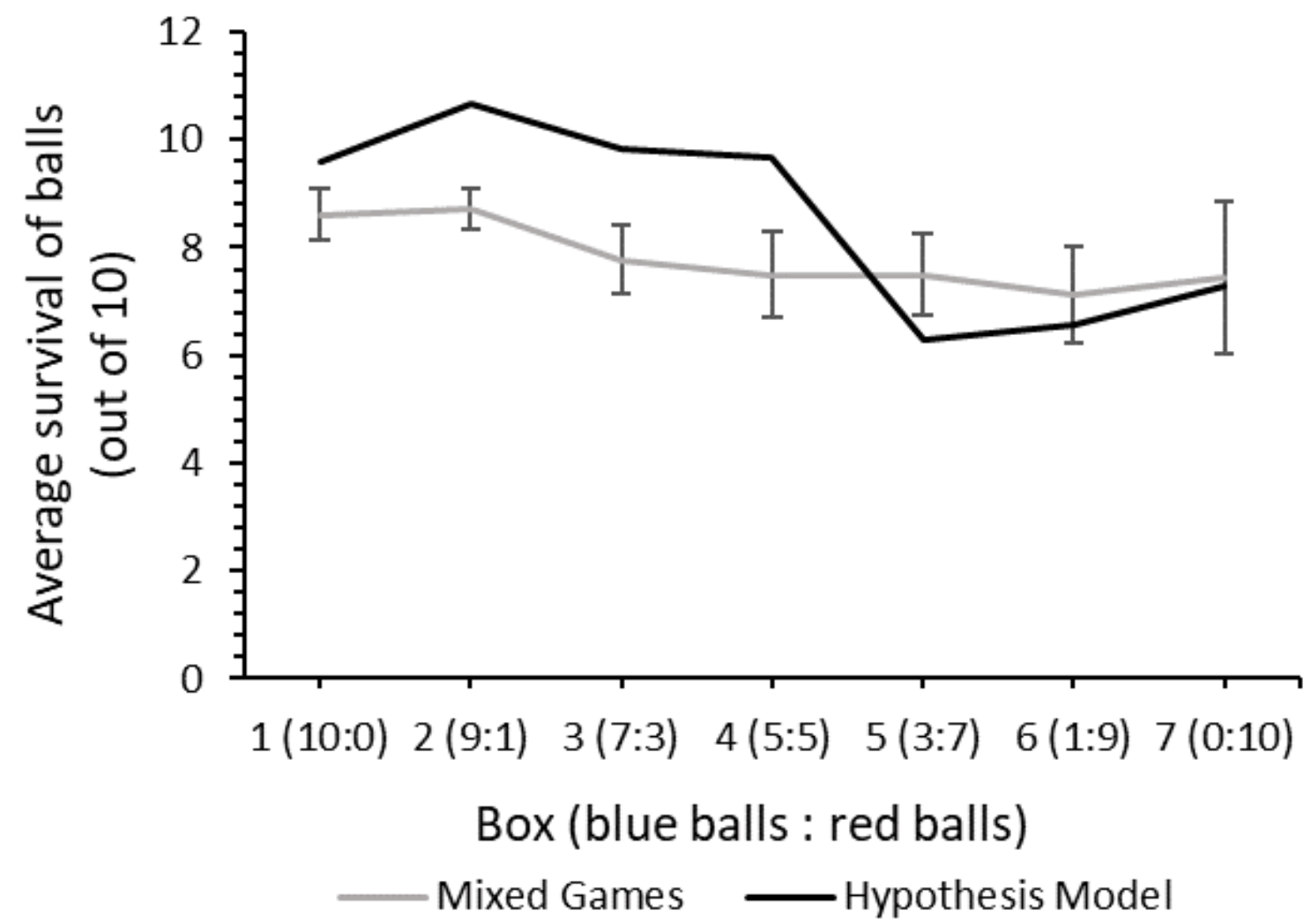

Figure 4.2. The expected average survival for each box based on the hypothesis model (black line) versus the observed average survival for each box in the trade-off games (grey line) with $95 \%$ confidence intervals.

Table 4.2. Mann-Whitney post-hoc analysis $p$-values for the pairwise comparisons of total survival at each pair of boxes in trade-off games. Bold face indicates values that are significant at the 0.05 significance level.

\begin{tabular}{c|cccccc}
\hline \hline Box (blue balls : red balls) & $1(10: 0)$ & $2(9: 1)$ & $3(7: 3)$ & $4(5: 5)$ & $5(3: 7)$ & $6(1: 9)$ \\
\hline $2(9: 1)$ & 0.790 & - & - & - & - & - \\
$3(7: 3)$ & 0.069 & $\mathbf{0 . 0 3 1}$ & - & - & - & - \\
$4(5: 5)$ & $\mathbf{0 . 0 4 2}$ & $\mathbf{0 . 0 1 8}$ & 0.796 & - & - & - \\
$5(3: 7)$ & $\mathbf{0 . 0 2 3}$ & $\mathbf{0 . 0 1 1}$ & 0.559 & 0.796 & - & - \\
$6(1: 9)$ & $\mathbf{0 . 0 0 9}$ & $\mathbf{0 . 0 0 3}$ & 0.366 & 0.529 & 0.747 & - \\
$7(0: 10)$ & 0.549 & 0.505 & 0.596 & 0.422 & 0.458 & 0.242 \\
\hline \hline
\end{tabular}


The composition of balls in Box 10:0 was the same in the security and trade-off games with ten yellow and ten blue balls. Additionally, the composition of balls in Box 0:10 was the same in the devaluation and trade-off games with ten red balls. It was therefore hypothesised that the survival at Box 10:0 would not differ between the security and trade-off games, and that the survival at Box 0:10 would not differ between the devaluation and trade-off games. At Box 0:10, there was no difference in survival between the devaluation games and the trade-off games (Devaluation mean $=6.55$, devaluation standard deviation $=3.91$; Trade-off mean $=8.61$, trade-off standard deviation 1.04). At Box 10:0, there was also no difference in survival between the security games and the trade-off games (Security mean $=9.5$, security standard deviation $=0.58$; Trade-off mean $=8.61$, trade-off standard deviation 1.04).

It was hypothesised that in the trade-off game, the presence of devaluation would result in all participants behaving similarly to each other, with a single dominant strategy being adopted by participants in response to devaluation. Instead, the coefficient of variation $(\mathrm{CV})$ in the survival of balls increased as devaluation increased from 12\% at Box 10:0 to $41 \%$ at Box 0:10 (Table 4.3).

Table 4.3. The average survival of balls, the standard deviation, and the coefficient of variation (CV) for survival at each box in the trade-off games.

\begin{tabular}{cccc}
\hline \hline Box (blue : red) & Survival Average & Standard Deviation & Coefficient of Variation $(\%)$ \\
\hline $1(10: 0)$ & 8.6 & 1.0 & 12.0 \\
$2(9: 1)$ & 8.7 & 0.8 & 9.5 \\
$3(7: 3)$ & 7.8 & 1.4 & 18.0 \\
$4(5: 5)$ & 7.5 & 1.7 & 23.0 \\
$5(3: 7)$ & 7.5 & 1.6 & 21.6 \\
$6(1: 9)$ & 7.1 & 1.9 & 27.2 \\
$7(0: 10)$ & 7.4 & 3.1 & 41.3 \\
\hline \hline
\end{tabular}




\subsubsection{Total selections and blue ball selections}

The total number of selections varied across boxes in the trade-off games (Kruskal-Wallis, $\mathrm{H}$ $(d f=6)=17.4, P=0.008$; Figure 4.3). The number of selections made at each box appears to follow a bell-curve shape with the most selections made at the central boxes, and fewer selections made towards each extreme. Post-hoc tests reveal that the number of selections at Box 10:0 was lower than Boxes 7:3 to 3:7, and the number of selections at Box 9:1 was also lower than Boxes 7:3 to 3:7 (Table 4.4). At the other extreme, the number of selections at Box 0:10 was lower than Boxes 7:3 to 3:7.

The number of blue balls selected also varied across boxes in the security-devaluation trade-off games $(\mathrm{H}(d f=5)=15.6, P=0.008$; Figure 4.3: Box 0:10 had no blue balls and so is excluded from this analysis). Post-hoc tests revealed that the average number of blue ball selections did not differ across the first five boxes, and that the number blue ball selections at Box 1:9 was significantly lower than all other boxes (Table 4.4). Each box contained a different number and proportion of blue balls (Figure 4.4). Therefore, the hunter-catch efficiency (total number of selections per blue ball selected) is also graphed (Figure 4.3, black line). The catch efficiency is relatively constant across the first four boxes but increases after Box 5:5. The most efficient box was Box 9:1 with the best catch efficiency (fewest balls selected per blue ball selected). 


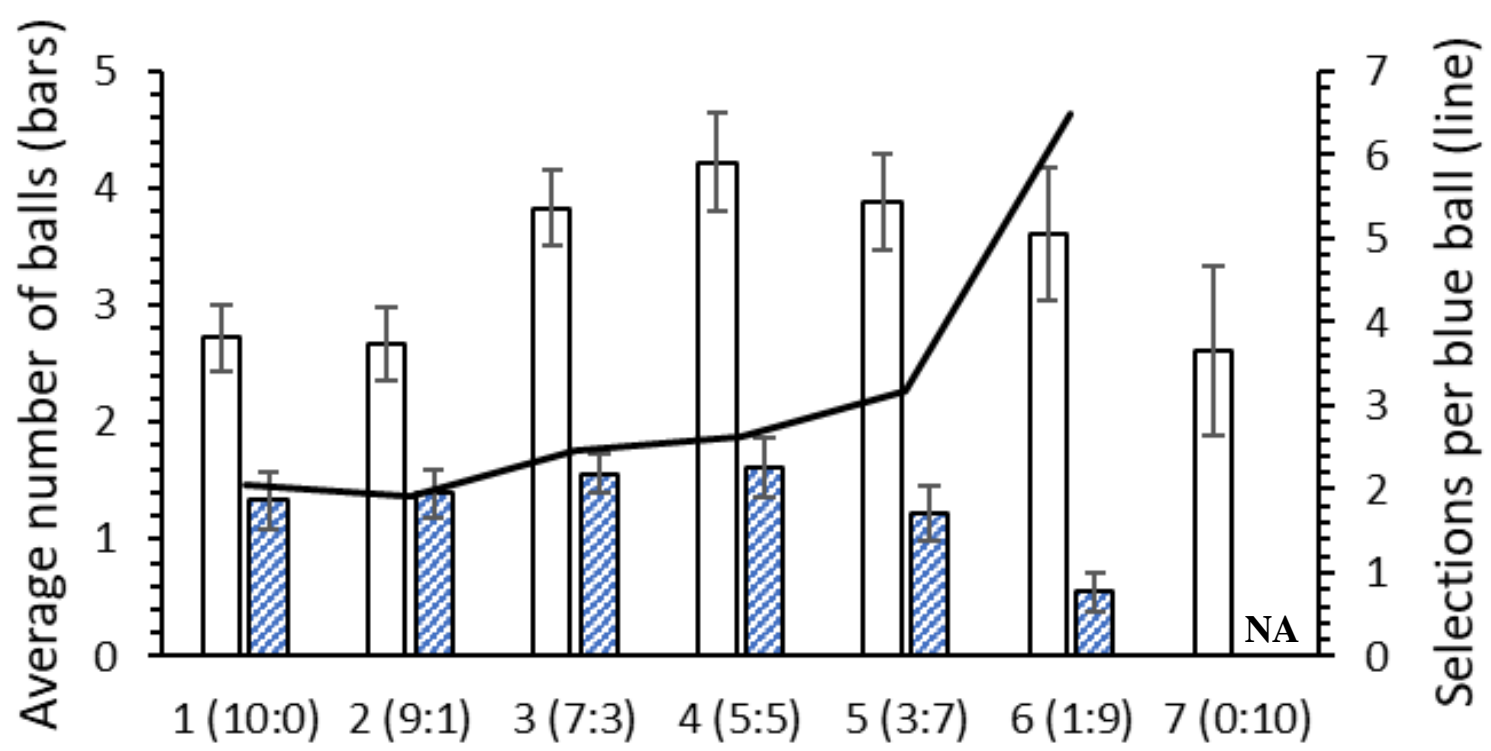

Box (blue balls : red balls)

\section{$\square$ Average Selections $\quad$ Average Blues Balls Selected}

Figure 4.3. The average number of balls selected, and the average number of blue balls selected at each box in trade-off games. The black line shows catch efficiency (the total number of selections per blue ball selected) and is represented by a secondary $y$-axis to the right. The $N A$ indicates that there were never blue balls present in Box 0:10, explaining the absence of data. Error bars are \pm 1 standard error. 


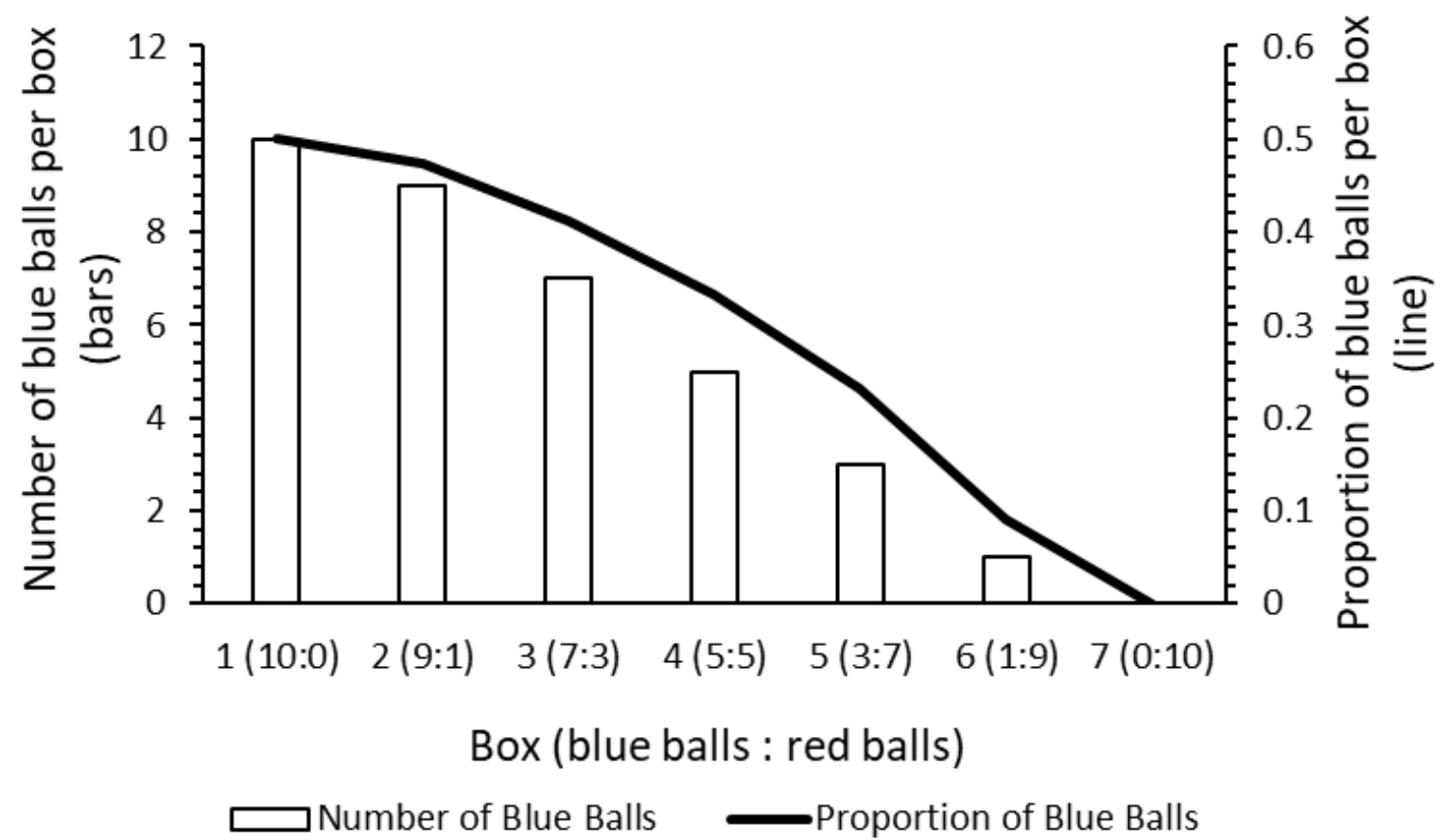

Figure 4.4. The number of blue balls placed in each box of the trade-off games (bars) and the proportion of blue balls starting in each box of the trade-off game (line). 
Table 4.4. Mann-Whitney post-hoc analysis $p$-values for the pairwise comparisons for each pair of boxes of (A) total selections in trade-off games, and (B) number of blue ball selections in trade-off games. Bold face indicates values that are significant at the 0.05 significance level.

(A)

\begin{tabular}{c|cccccc}
\hline \hline Box (blue : red) & $1(10: 1)$ & $2(9: 1)$ & $3(7: 3)$ & $4(5: 5)$ & $5(3: 7)$ & $6(1: 9)$ \\
\hline $2(9: 1)$ & 0.870 & - & - & - & - & - \\
$3(7: 3)$ & $\mathbf{0 . 0 1 9}$ & $\mathbf{0 . 0 2 8}$ & - & - & - & - \\
$4(5: 5)$ & $\mathbf{0 . 0 0 5}$ & $\mathbf{0 . 0 0 6}$ & 0.594 & - & - & - \\
$5(3: 7)$ & $\mathbf{0 . 0 2 6}$ & $\mathbf{0 . 0 3 7}$ & 0.974 & 0.573 & - & - \\
$6(1: 9)$ & 0.271 & 0.274 & 0.450 & 0.254 & 0.501 & - \\
$7(0: 10)$ & 0.247 & 0.310 & $\mathbf{0 . 0 2 5}$ & $\mathbf{0 . 0 1 4}$ & $\mathbf{0 . 0 3 4}$ & 0.099 \\
\hline \hline
\end{tabular}

(B)

\begin{tabular}{c|ccccc}
\hline \hline Box (blue : red) & $1(10: 1)$ & $2(9: 1)$ & $3(7: 3)$ & $4(5: 5)$ & $5(3: 7)$ \\
\hline $2(9: 1)$ & 0.765 & - & - & - & - \\
$3(7: 3)$ & 0.393 & 0.594 & - & - & - \\
$4(5: 5)$ & 0.449 & 0.593 & 0.946 & - & - \\
$5(3: 7)$ & 0.726 & 0.411 & 0.144 & 0.226 & - \\
$6(1: 9)$ & $\mathbf{0 . 0 1 8}$ & $\mathbf{0 . 0 0 5}$ & $<\mathbf{0 . 0 0 0}$ & $\mathbf{0 . 0 0 3}$ & $\mathbf{0 . 0 2 9}$ \\
\hline \hline
\end{tabular}




\subsubsection{Hunter treatment of devalued balls}

When a red ball was selected the participant could choose to either keep it, return it or discard

it. The outcome of red balls differed depending on the value of the red ball $\left(\chi^{2}{ }_{4}=278.8\right.$, $\mathrm{N}=560, P<0.001$, Figure 4.5). Post-hoc tests revealed that when red balls were worthless, a lower number were kept and returned, and a higher number discarded (Table 4.5).

Conversely, when red balls were worth 50 cents, a higher number were kept and returned, and a lower number discarded. There was no difference between the numbers of red balls kept, returned and discarded when red balls were worth 20 cents. 


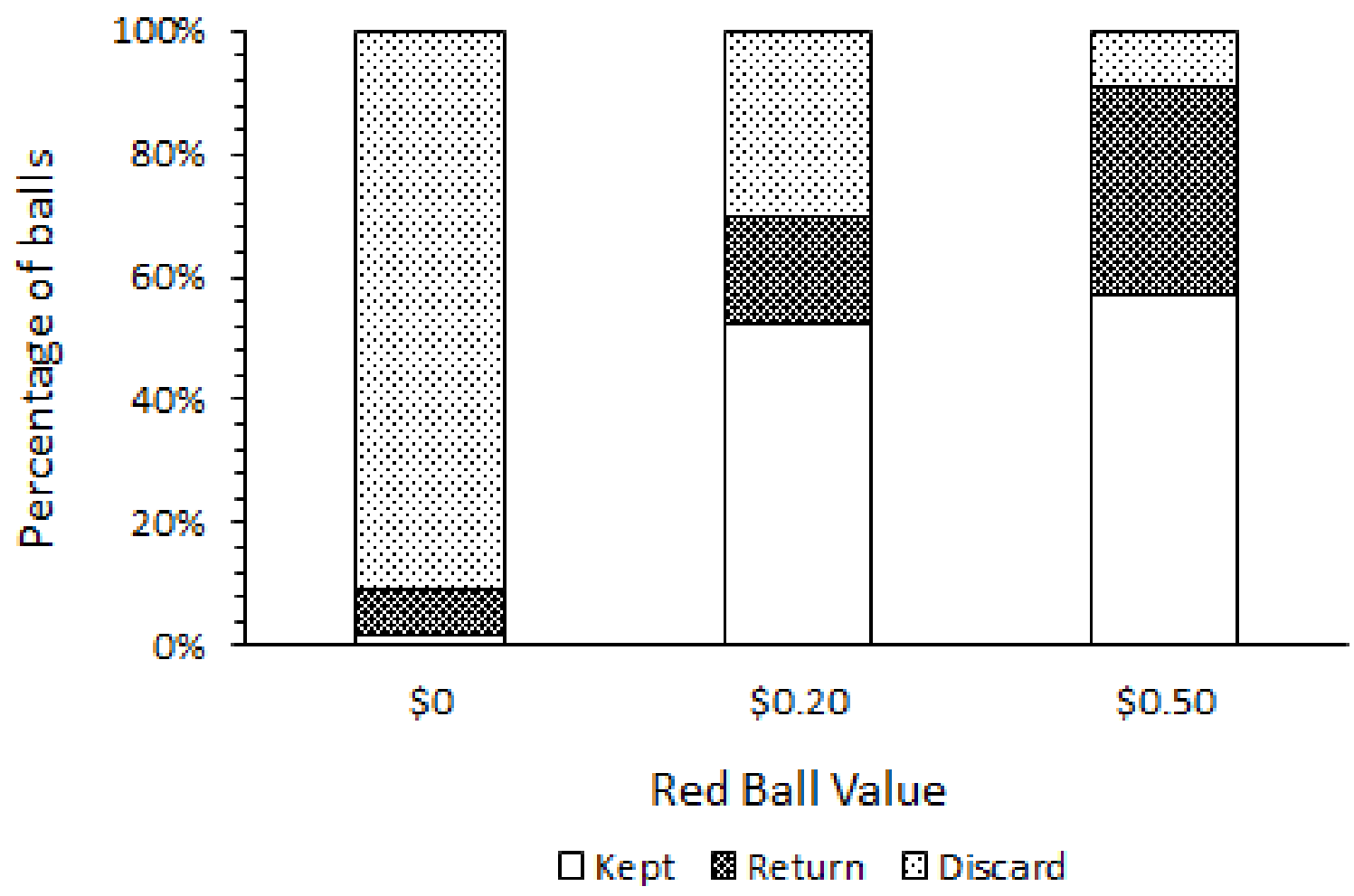

Figure 4.5. The percentage of red balls kept, returned and discarded for each of the red ball values.

Table 4.5. The results of the post-hoc tests for the chi-square test of red ball outcome and red ball value. The values presented are residuals with a Bonferroni correction. The cut-off at the 0.05 significance level is \pm 2.773 . The significant values are marked in bold face.

\begin{tabular}{c|ccc}
\hline \hline & \multicolumn{3}{c}{ Red Ball Value } \\
& $\$ 0$ & $\$ 0.20$ & $\$ 0.50$ \\
\hline Kept & $\mathbf{- 1 0 . 2 7}$ & 1.40 & $\mathbf{7 . 4 2}$ \\
Return & $\mathbf{- 4 . 9 4}$ & -2.00 & $\mathbf{5 . 6 3}$ \\
Discard & $\mathbf{1 6 . 0 7}$ & 0.39 & $\mathbf{- 1 3 . 6 0}$ \\
\hline \hline
\end{tabular}


The outcome of red balls also differed between the devaluation and trade-off games $\left(\chi_{2}^{2}\right.$ $=95.7, \mathrm{~N}=560, P<0.001$; Figure 4.6). Post-hoc tests revealed that in the devaluation games without blue balls, fewer red balls were kept, and more were returned and discarded (Table 4.6). Conversely, in trade-off games with blue balls, fewer red balls were returned and discarded, and more were kept.

Because both chi-square tests produced results which rejected the null hypothesis, logistic mixed-effect models were used to test for an interaction between red ball value and game type. Red ball value $(P<0.001)$, game type $(P<0.001)$ and their interaction $(P<$ 0.001) proved significant in determining whether a red ball was kept. However, only red ball value proved significant in determining whether a red ball that was not kept was returned or discarded $(P=0.003)$. 


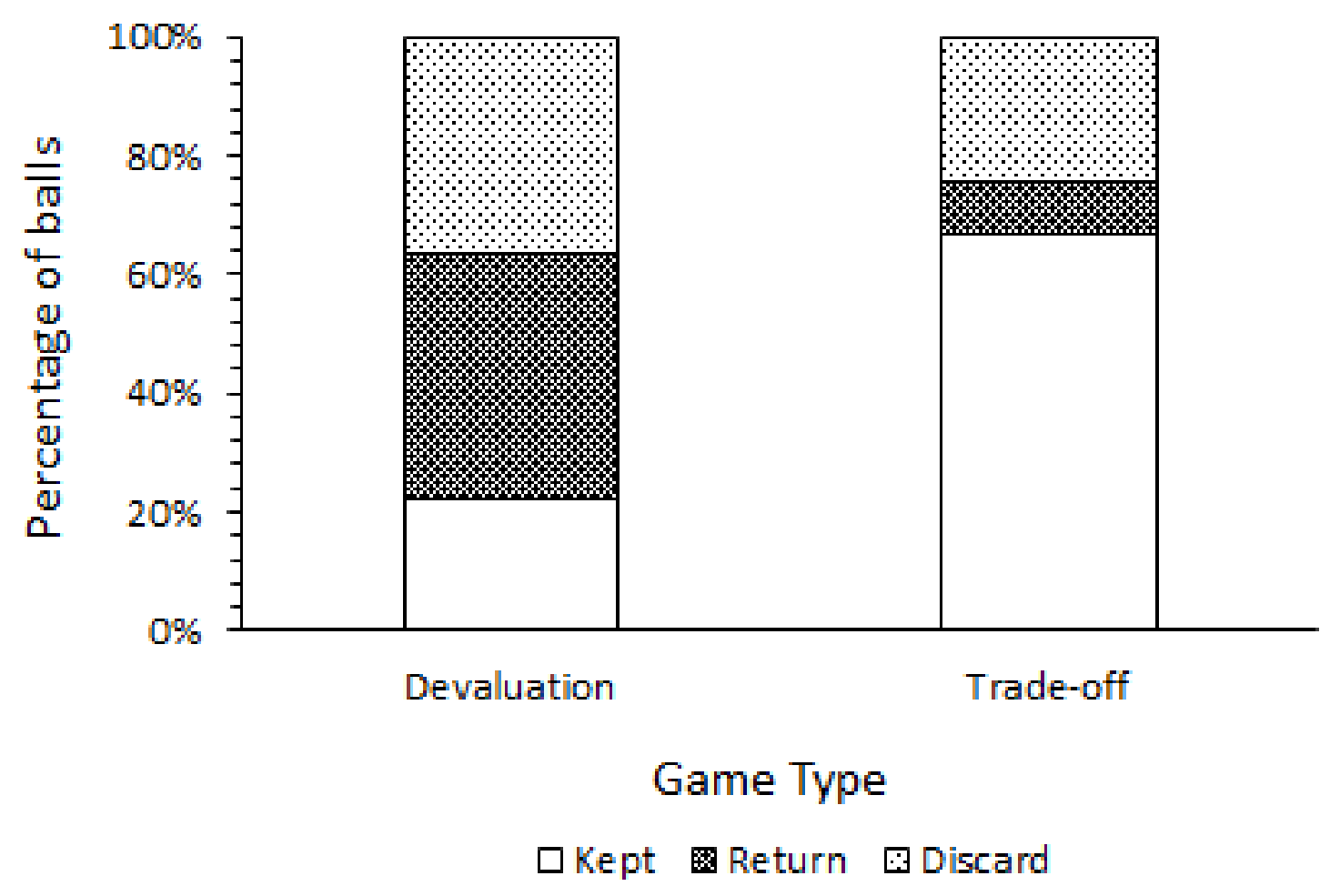

Figure 4.6. The percentage of red balls kept, returned and discarded in devaluation and trade-off games.

Table 4.6. The results of the post-hoc tests for the chi-square test of red ball outcome and game type. The values presented are residuals with a Bonferroni correction. The cut-off at the 0.05 significance level is \pm 2.638 . The significant values are marked in bold face.

\begin{tabular}{c|cc}
\hline \hline & \multicolumn{2}{c}{ Game Type } \\
& Devaluation & Trade-off \\
\hline Kept & $\mathbf{- 9 . 5 5}$ & $\mathbf{9 . 5 5}$ \\
Return & $\mathbf{6 . 9 2}$ & $\mathbf{- 6 . 9 2}$ \\
Discard & $\mathbf{3 . 8 3}$ & $\mathbf{- 3 . 8 3}$ \\
\hline \hline
\end{tabular}


The outcome of red balls also differed between the five sites where the games were played $\left(\chi^{2}{ }_{8}=192.5, \mathrm{~N}=560, P<0.001\right.$; Figure 4.7$)$. These differences are potentially explained by the socio-economic status of the separate communities (Figure 4.8). Post-hoc tests revealed that in Petone, a higher number of red balls were kept, and a lower number were returned or discarded (Table 4.7). In Upper Hutt a higher number were kept, and a lower number were discarded. In Aro, Karori and Seatoun, a lower number of red balls were kept, and a higher number were discarded.

Again, because both chi-square tests produced results which rejected the null hypothesis, logistic mixed-effect models were used to test for an interaction between red ball value and game type. Only Petone proved significant in determining whether a red ball was kept $(P=0.047)$. None of the fixed effects or interactions proved significant in determining whether a red ball that was not kept was returned or discarded. 


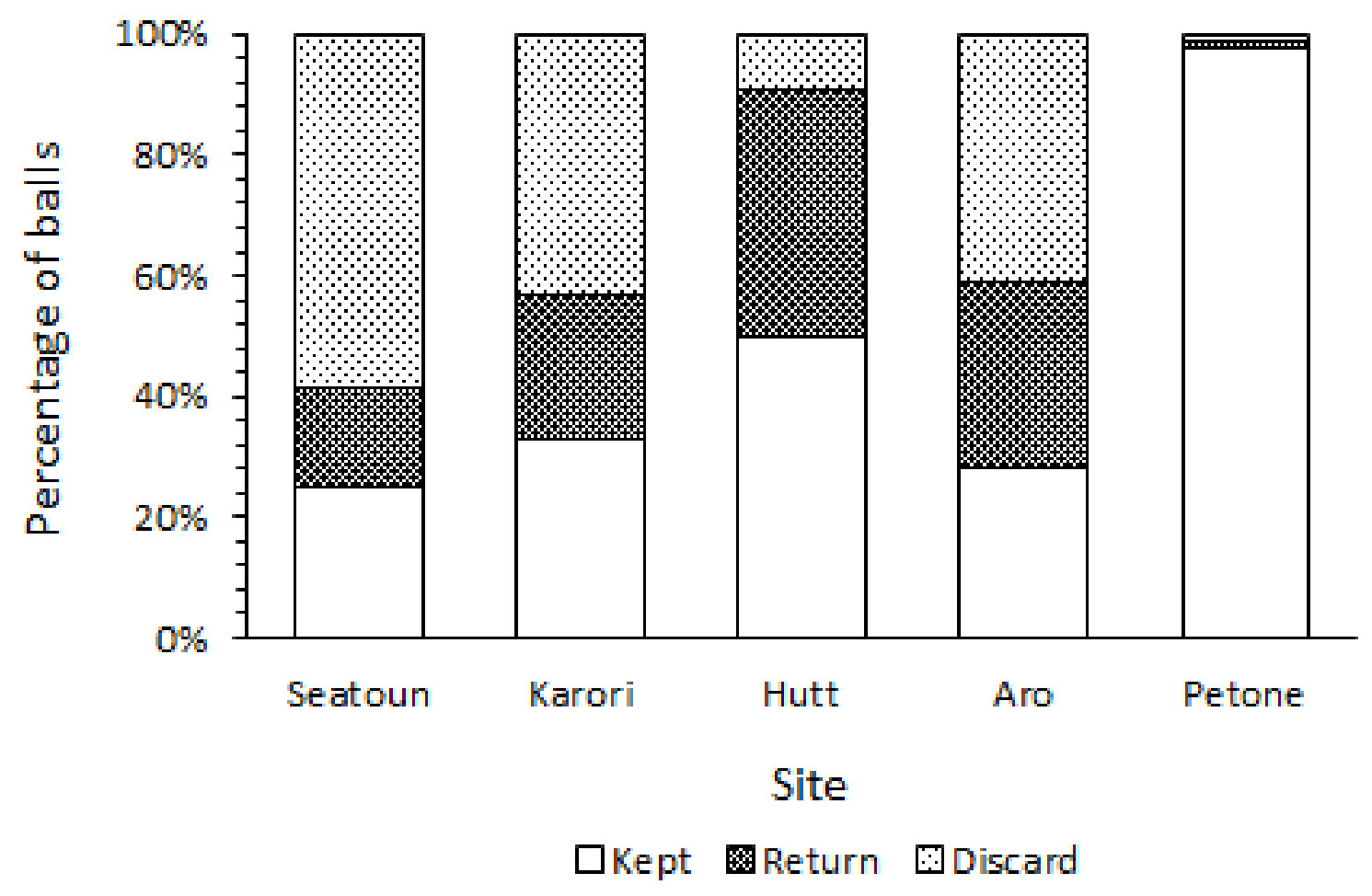

Figure 4.7. The percentage of red balls kept, returned and discarded for each of the five sites. The suburbs are ordered from least deprived (left) to most deprived (right), as per Figure 4.8.

Table 4.7. The results of the post-hoc tests for the chi-square test of red ball outcome and site. The sites are ordered from least deprived (left) to most deprived (right). The values presented are residuals with a Bonferroni correction. The cut-off at the 0.05 significance level is \pm 2.935 . The significant values are marked in bold face.

\begin{tabular}{c|ccccc}
\hline \hline & \multicolumn{5}{c}{$\underline{\text { Site }}$} \\
& Seatoun & Karori & Upper Hutt & Aro Valley & Petone \\
\hline Kept & $\mathbf{- 3 . 9 8}$ & $\mathbf{- 2 . 9 6}$ & 1.34 & $\mathbf{- 4 . 4 2}$ & $\mathbf{1 0 . 5 5}$ \\
Return & -2.09 & -0.37 & $\mathbf{5 . 1 1}$ & 1.47 & $\mathbf{- 5 . 6 6}$ \\
Discard & $\mathbf{6 . 4 0}$ & $\mathbf{3 . 6 2}$ & $\mathbf{- 6 . 4 1}$ & $\mathbf{3 . 4 5}$ & $\mathbf{- 6 . 1 4}$ \\
\hline \hline
\end{tabular}



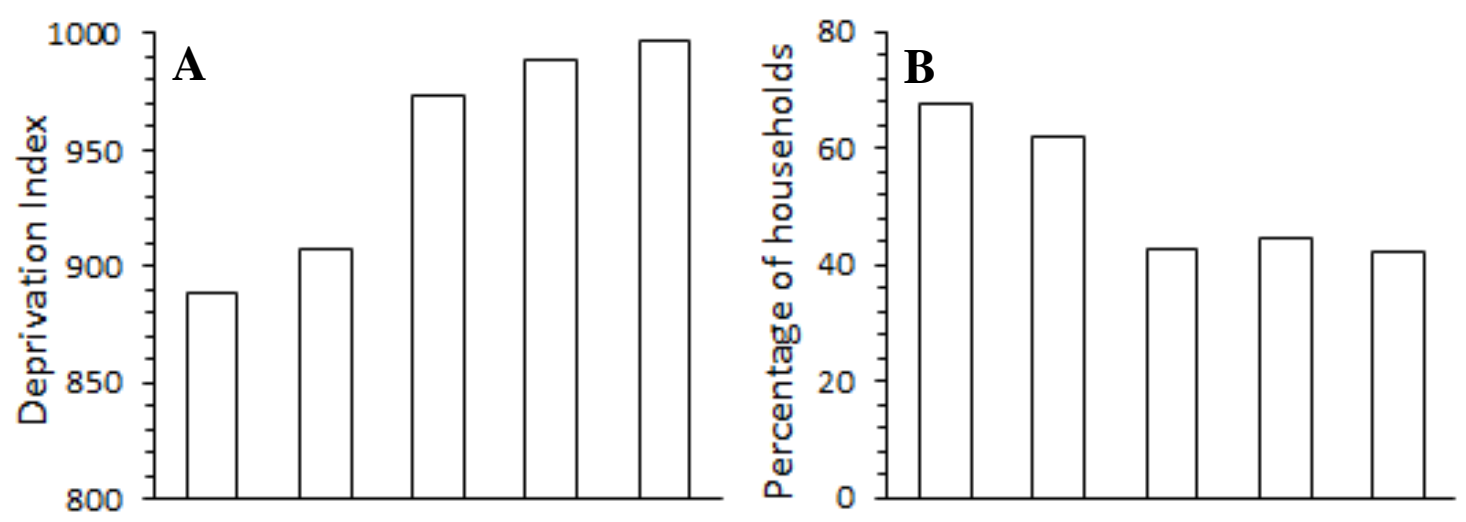

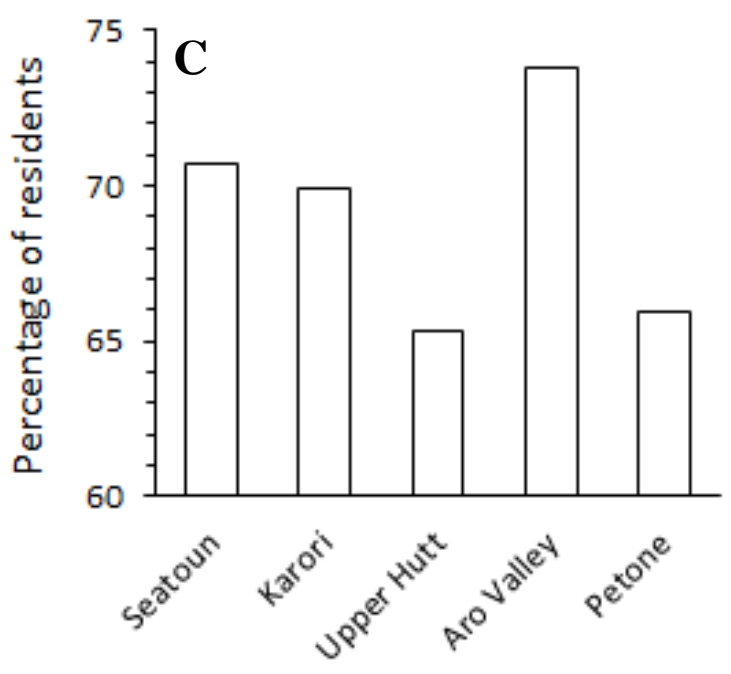

Suburb

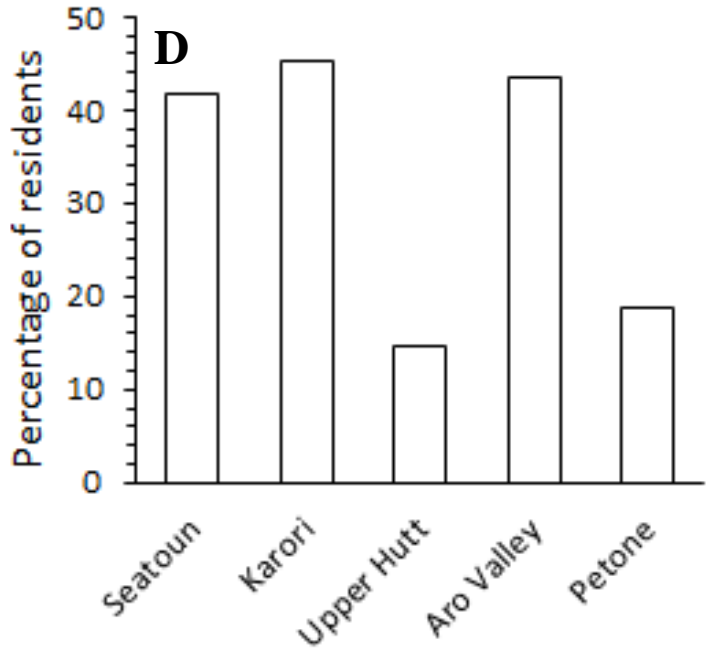

Suburb

Figure 4.8. New Zealand census data of the percentage of residents in each

suburb where games were conducted (profile.idnz.co.nz, 2018a;

profile.idnz.co.nz., 2018b).

$\mathrm{A}=$ deprivation index $\mathrm{B}=$ percentage of households with high income (> \$70,000); $\mathrm{C}=$ labour force participation percentage; $\mathrm{D}=$ university education percentage.

The household income threshold of $\$ 70,000$ is the threshold used by profile.idnz.co.nz. 
Eighteen trade-off games were completed totalling 180 participants, and surveys were received from 171 (95\%) of those. Of the 180 participants, 16 won (9\%) and 164 lost (91\%). Surveys were received from all 16 participants who won (100\%) and 155 of the 164 participants who lost (95\%).

- A larger number of winners stated that they cooperated with other participants (19\%) compared to the number of losers who stated that they cooperated (6\%). However, this difference was not statistically significant (Fisher's exact test: $P=0.093$ ).

- When comparing the participants who kept red balls with those who did not, winners were more likely than losers to keep a red ball (56\% and 32\% respectively; Fisher's exact test: $P=0.024)$.

- Of the winning and losing participants who kept red balls, more winners did so because they deemed them valuable enough or saw the benefit in accumulating low value balls ( $44 \%$ and $16 \%$ respectively; Fisher's exact test: $P=0.017)$.

- When comparing the participants whose strategies were to select from low risk boxes with those who selected from high risk boxes, more winners selected from low risk boxes (75\% and 34\% respectively; Fisher's exact test: $P=0.048)$.

- There was no significant difference in the number of winners and losers whose strategy was to select form moderate risk-reward boxes (19\% and 25\% respectively; Fisher's exact test: $P=0.764)$.

- Winning and losing participants who selected a red ball returned red balls at the same rate (both 9\%; Fisher's exact test: $P=1.00$ ).

- Winning and losing participants who selected a red ball discarded red balls at a statistically similar rate (9\% and $21 \%$ respectively; Fisher's exact test: $P=0.684$ ).

Those who won money were more inclined to select from low risk boxes, instead of selecting from high risk boxes to get yellow balls. Additionally, those who won were more inclined to keep devalued red balls as opposed to returning them or discarding them. It is also possible that those who cooperated with other participants had a better chance of winning. 


\subsubsection{Dynamic games}

Based on the results of the devaluation, security and trade-off lucky dip games, it was hypothesised that if guards were behaving rationally or optimally, they would adopt security strategies by placing high numbers of blue balls in their boxes. Most often, the initial strategy used by guards was to put a high number of blue balls in their box. The average number of blue balls that guards placed in their boxes was 8.5 (out of 10 ; standard deviation $=1.9$ ), and the highest number of red balls that a guard included in their box was five. The number of selections at each box increased with a weak correlation as the number of red balls initially placed in the box decreased $\left(\mathrm{R}=0.282 ; \mathrm{R}^{2}=0.080\right)$. This shows that hunters targeted devalued boxes. Even when the value of the red balls was reduced from 50 cents to 20 cents hunter participants still targeted devalued boxes ( 50 cents: $R=0.255, R^{2}=0.065 ; 20$ cents: $R$ $\left.=0.492, \mathrm{R}^{2}=0.242\right)$. The number of balls surviving in a box declined with a moderate correlation as the number of red balls initially placed in the box increased $\left(R=-0.376 ; R^{2}=\right.$ 0.141). The number of blue balls selected from a box increased with a weak correlation as the number of blue balls initially placed in the box increased $\left(\mathrm{R}=0.243 ; \mathrm{R}^{2}=0.059\right)$. 


\subsection{Scavenger-hunt games}

In all games, devalued stakes found by the participants were all removed from their standing position and laid on the ground so that they were removed from the game. Therefore, there was no significant difference in how participants responded to devalued items across the three devaluation levels. There was no significant difference in the number of stakes 'killed' across the three devaluation levels $\left(\chi^{2}{ }_{2}=4.7, \mathrm{~N}=80, P=0.094\right.$; Figure 4.9). Participants' Likert scale motivational scores did not differ significantly between devaluation levels (Kruskal-Wallis, $\mathrm{H}(d f=2)=1.1, P=0.571$; Figure 4.10). 


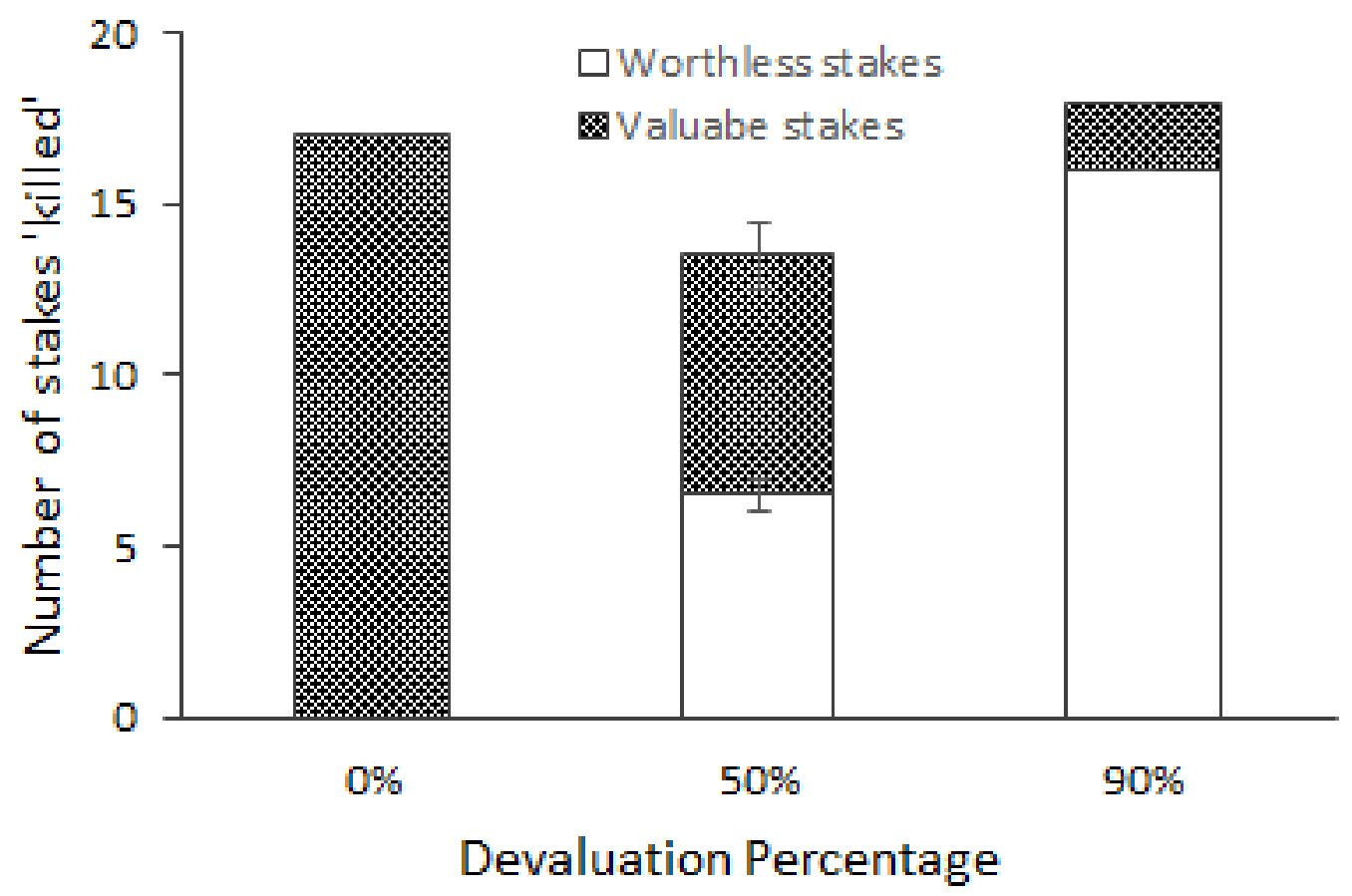

Figure 4.9. The average number of stakes 'killed' (delivered or laid down) in the scavenger-games of each devaluation level. Error bars are \pm 1 standard error. 


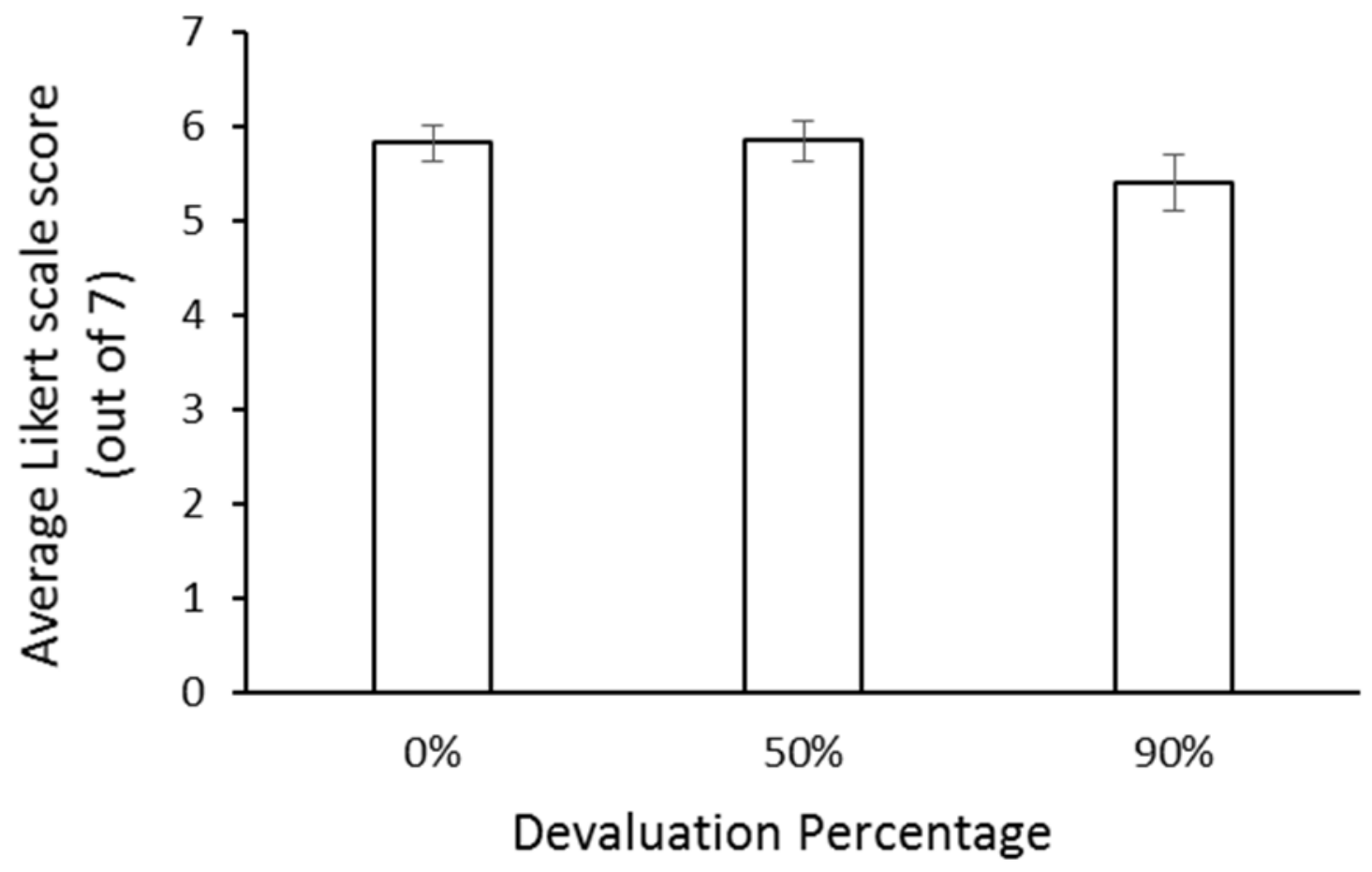

Figure 4.10. The average Likert scale score of participant motivation for each devaluation level ( $1=$ not motivated; $7=$ highly motivated $)$. Error bars are \pm 1 standard error. 
There was no difference in the average number of steps per participant pair across the three devaluation levels (ANOVA, $\mathrm{F}_{2,18}=1.0, P=0.382$; Figure 4.11). There was a difference in the average number of steps per participant pair across the three game stages (ANOVA, $\mathrm{F}_{2,49}=5.3, P=0.008$; Figure 4.12). Post-hoc tests revealed that the average steps during game stage three was significantly lower than stages one and two (Table 4.8). Stages one and two did not differ. 


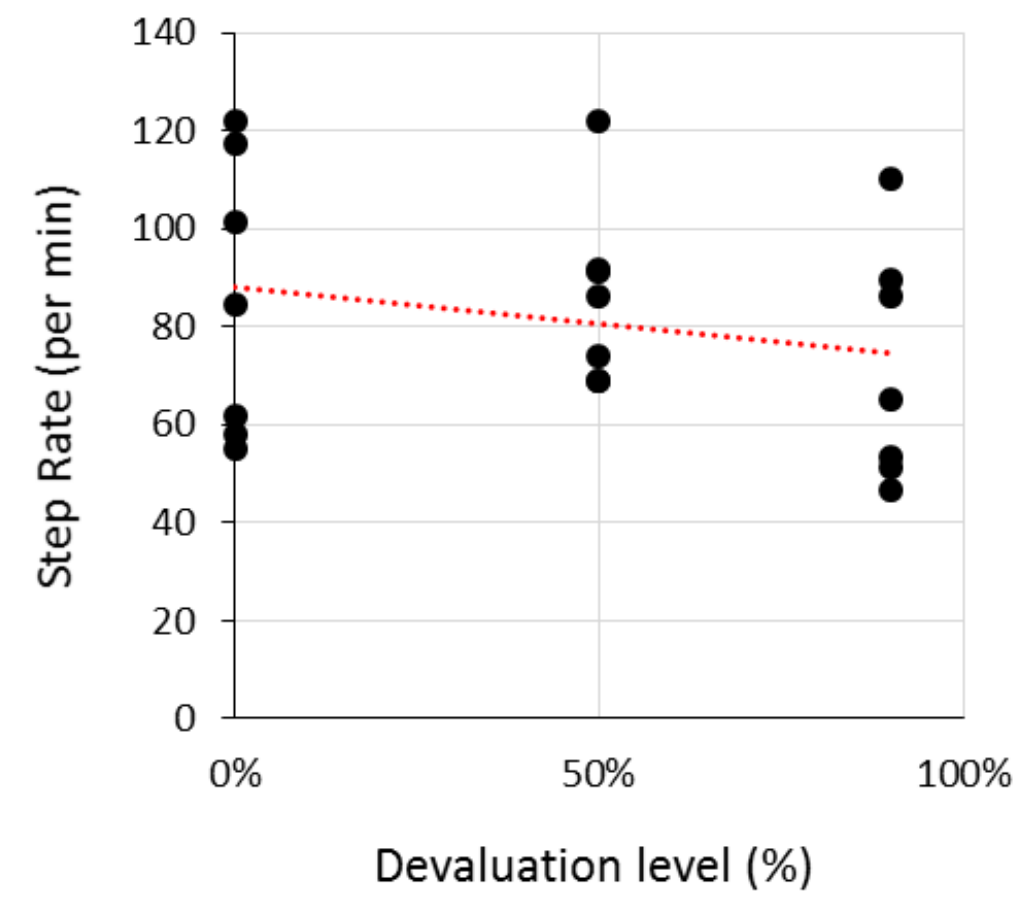

Figure 4.11. The average number of steps per participant pair at each devaluation level.

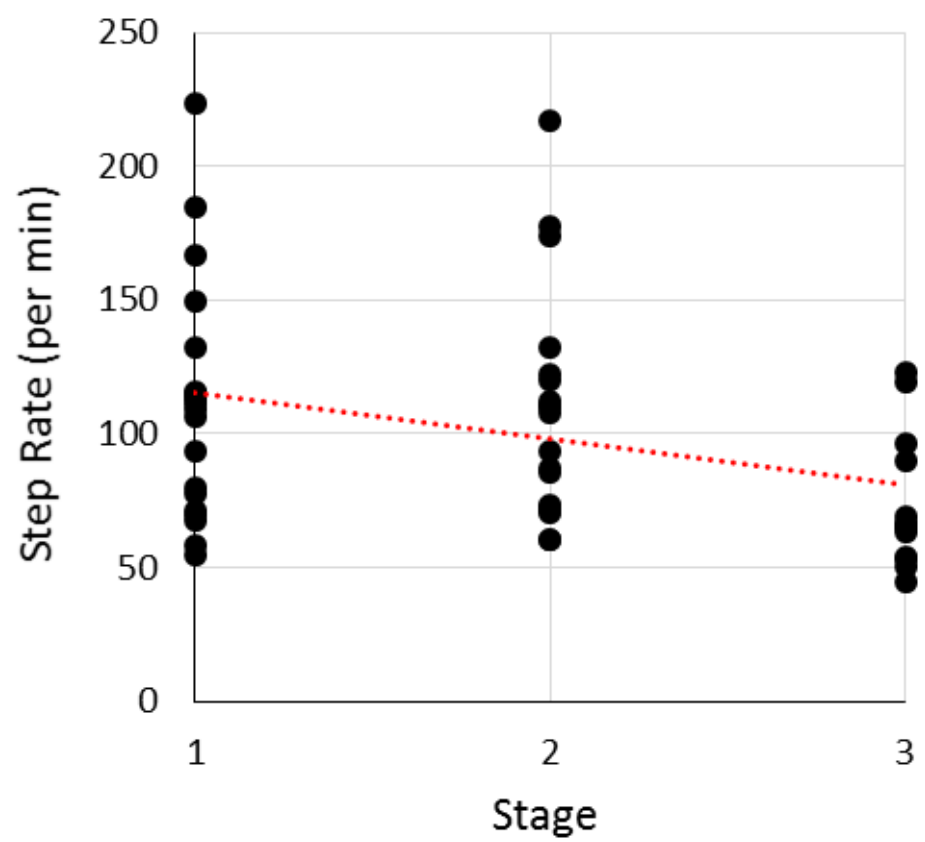

Figure 4.12. The average number of steps per participant pair during each game stage, with all devaluation levels combined. 
Table 4.8. Post-hoc Tukey test results for the ANOVA of the average number of steps per participant pair across the three game stages. Bold face indicates values that are significant at the 0.05 significance level.

\begin{tabular}{c|c}
\hline Stage Comparison & $p$-value \\
\hline Stage 1 - Stage 2 & 0.987 \\
Stage 1 - Stage 3 & $\mathbf{0 . 0 1 2}$ \\
Stage 2 - Stage 3 & $\mathbf{0 . 0 1 9}$ \\
\hline \hline
\end{tabular}




\subsection{Online survey}

The Bradley-Terry model used to analyse the pairwise comparison data revealed that Park 1 with no devaluation was consistently the least popular when paired with all three other parks (Figure 4.13; Table 4.9). There was no significant difference in preferences between Parks 2 (50\% devaluation), 3 (75\% devaluation) and 4 (90\% devaluation).

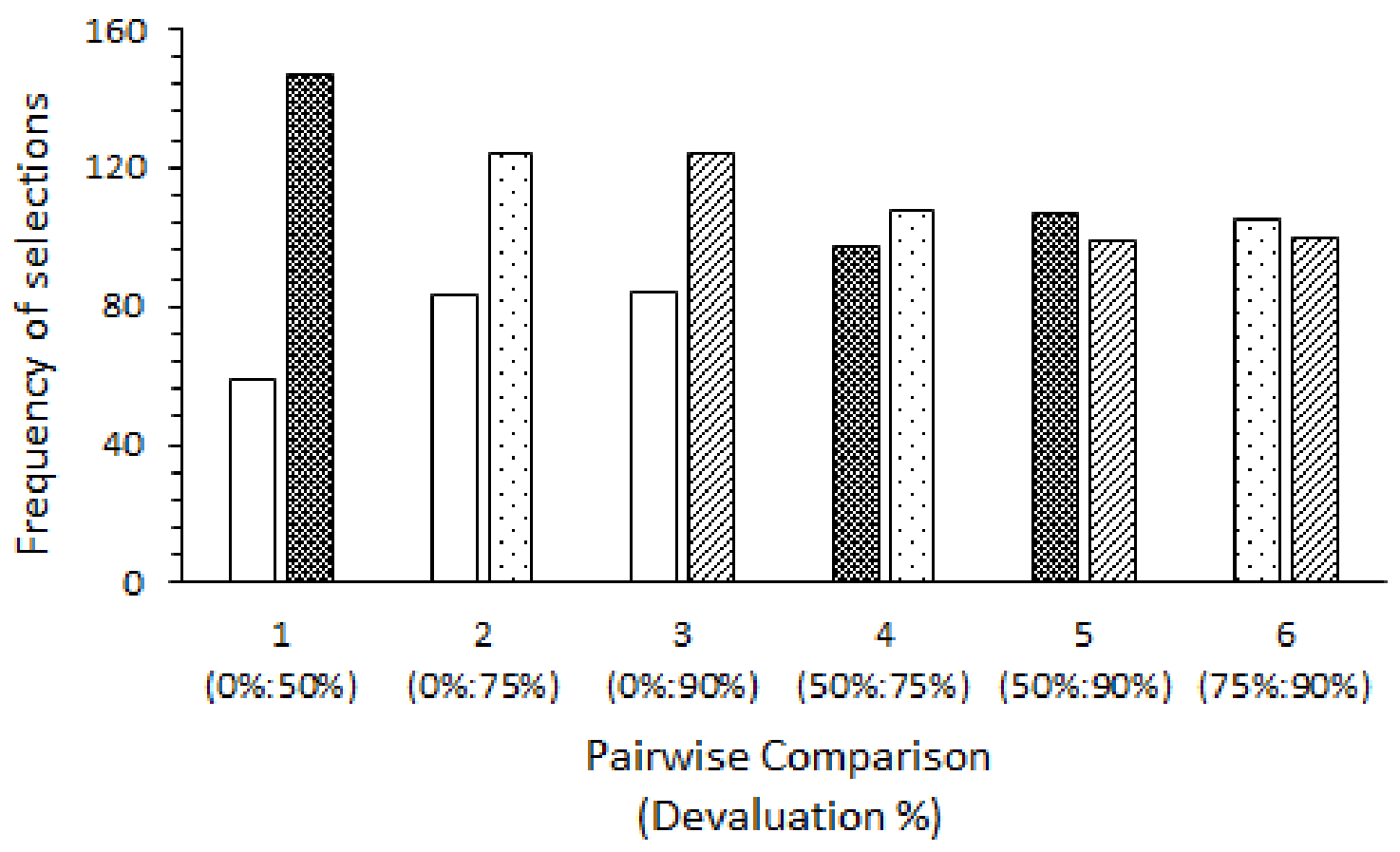

Figure 4.13. The number of times each park was selected in each of the six pairwise comparisons. Each of the four parks with its own devaluation percentage $(0 \%, 50 \%, 75 \%$ and $90 \%)$ is distinguished by a unique bar pattern.

Table 4.9. The Bradley-Terry model output $p$-values for each park pairwise comparison. Bold face indicates values that are significant at the 0.05 significance level.

\begin{tabular}{c|ccc}
\hline \hline Park (Devaluation \%) & $1(0 \%)$ & $2(50 \%)$ & $3(75 \%)$ \\
\hline $2(50 \%)$ & $<\mathbf{0 . 0 0 1}$ & - & - \\
$3(75 \%)$ & $<\mathbf{0 . 0 0 1}$ & 0.481 & - \\
$4(90 \%)$ & $<\mathbf{0 . 0 0 1}$ & 0.146 & 0.453 \\
\hline \hline
\end{tabular}


Ranking and park are not independent $\left(\chi_{9}^{2}=197.3, \mathrm{~N}=636, P<0.001\right.$; Figure 4.14). Post-hoc tests revealed that Park 1 (no devaluation) was the least preferred, receiving a high number of fourth ranks, and a lower number of second and third ranks (Table 4.10). Park 2 (50\% devaluation) received a low number of fourth ranks and a higher number of third ranks, while Park 3 (75\% devaluation) received low numbers of first and fourth ranks, and the highest number of second ranks. Park 4 (90\% devaluation) received high numbers of extreme ranks (one and four) and low numbers of moderate ranks (two and three) (Table 4.10; Figure 4.14). 


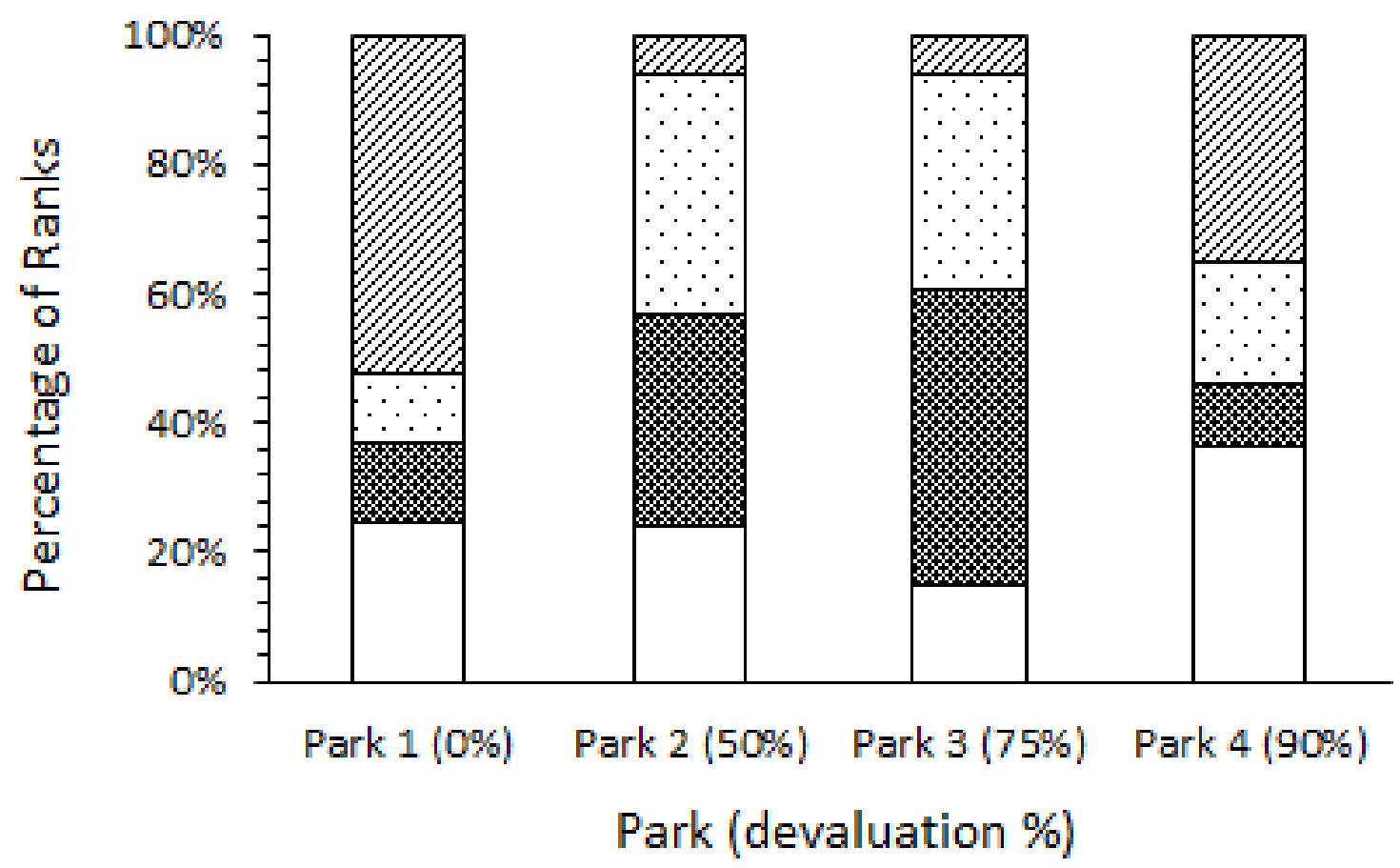

\section{口Rank 1 咚Rank 2 Rank 3 『Rank 4}

Figure 4.14. The percentage of each rank score $(1=$ most preferred; $4=$ least preferred) received by each of the four parks. $\mathrm{N}=159$.

Table 4.10. The results of the post-hoc tests for the chi-square test of park and ranking. The values presented are residuals with a Bonferroni correction. The cut-off at the 0.05 significance level is \pm 2.955 . The significant values are marked in bold face.

\begin{tabular}{l|cccc}
\hline \hline & Rank 1 & Rank 2 & Rank 3 & Rank 4 \\
\hline Park 1 & -0.16 & $\mathbf{- 4 . 1 8}$ & $\mathbf{- 4 . 8 1}$ & $\mathbf{9 . 1 5}$ \\
Park 2 & -0.37 & 2.59 & $\mathbf{4 . 0 7}$ & $\mathbf{- 6 . 2 9}$ \\
Park 3 & $\mathbf{- 3 . 3 3}$ & $\mathbf{6 . 8 2}$ & 2.80 & $\mathbf{- 6 . 2 9}$ \\
Park 4 & $\mathbf{3 . 8 6}$ & $\mathbf{- 5 . 2 3}$ & -2.06 & $\mathbf{3 . 4 4}$ \\
\hline \hline
\end{tabular}


For Section 2 of the survey, whether respondents chose to return or discard was not independent of whether there were more yearlings or stags in the population $\left(\chi^{2}{ }_{1}=9.0\right.$, $\mathrm{N}=767, P=0.003$; Figure 4.15). When there was a higher proportion of yearlings in the presented population, more respondents selected to discard the yearling. Alternatively, when there was a higher proportion of mature stags in the presented population, more respondents selected to return the yearling.

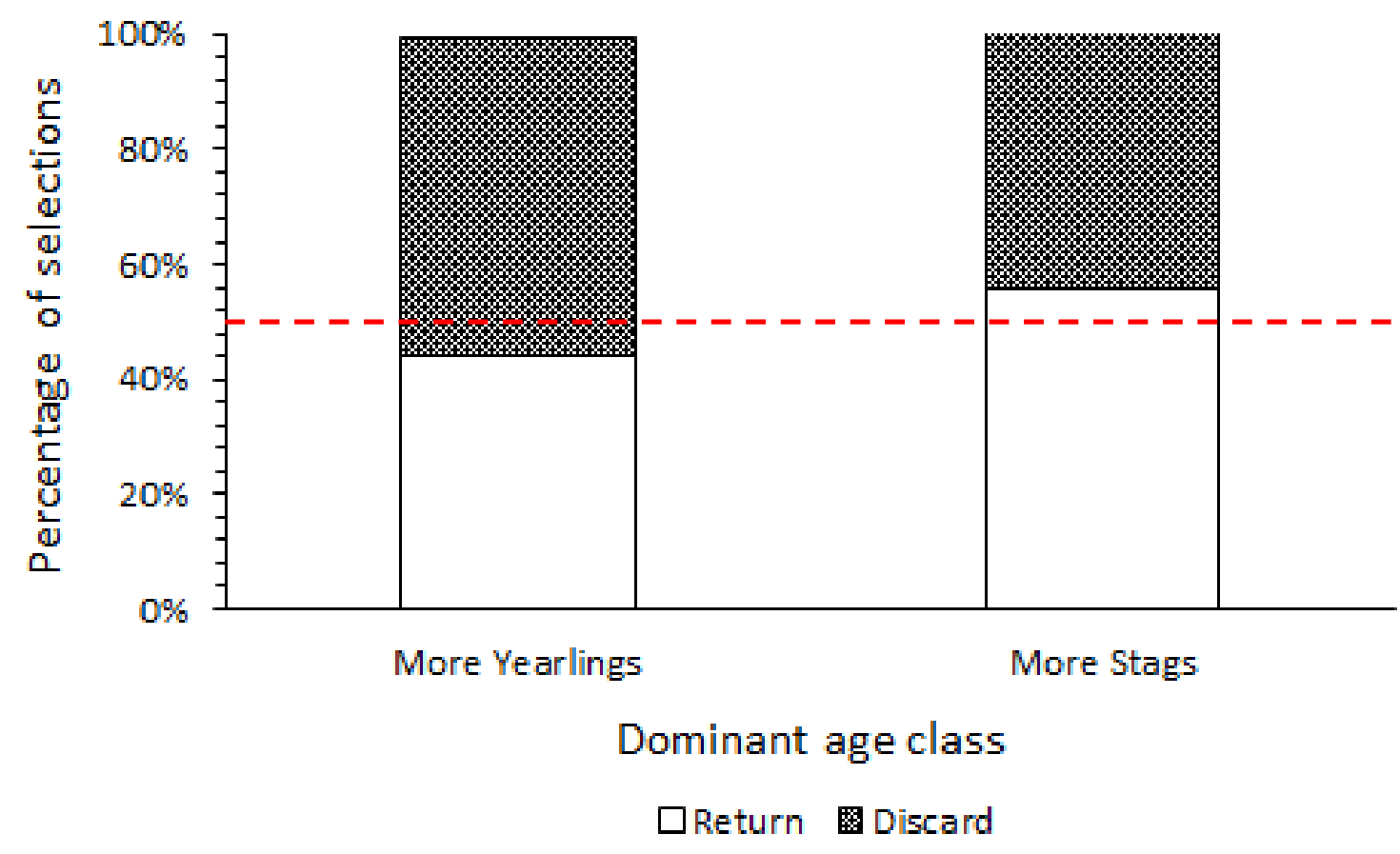

Figure 4.15. The percentage of respondents who selected return and discard when there were more yearlings in the presented populations, and more stags in the presented populations. The red dashed line marks the $50 \%$ threshold. 



\section{Discussion}

Two serious games and a scenario choice-based survey were used to study human hunting behaviour. Lucky-dip games were rapid, highly replicated indoor games. They were used to test the impact of variation in devaluation and security strategies across multiple populations. These games also enabled the investigation of how the magnitude of devaluation influences outcomes. Scavenger-hunt games were conducted over a three-hour period and a larger area. These games elicited more complex behaviours and interactions between participants enabling the study of these behaviours. Finally, the online scenario choice-based survey enabled recreational hunters to participate in the research by having them respond to hypothetical circumstances that were similar to those posed to participants in the lucky-dip and scavenger-hunt games. Each of the three research methods presented similar scenarios but used different formats to test the ideas amongst a diverse population of people. This allowed for the examination behavioural nuances that are important when trying to understand the complexities of mitigating and managing the IWT. The results across all three methods were consistent and complementary, which provides confidence in the conclusions reached.

The main finding is that when combined with security, devaluation failed to increase commodity survival. In the security and devaluation games, increasing either security or devaluation independently improved survival. This shows that both security and devaluation succeed independently and supports the assumption that hunters avoid risk and hunt for higher-value commodities. This potentially explains why proponents of devaluation are committed to it and have invested in developing new and innovative devaluation methods. However, the IWT is complex and nuanced. Hunting prevention strategies are not implemented independently, few parks are geographically isolated but instead form multipark systems, and human behaviour varies. When the games presented participants with a trade-off between devaluation and security, survival decreased as devaluation increased. Five reasons why devaluation failed to protect commodities in the games are discussed below, which provides insight into why devaluation of rhino has often failed to protect rhino. 


\subsection{Why did devaluation not succeed?}

\subsubsection{Devaluation vs. Security}

When combined with security, devaluation failed to prevent the harvesting of balls because risk was a greater deterrent such that participants harvested devalued items instead of taking greater risks to find higher-value items. Even though survival increased after Box 5:5 in the devaluation games due to increasing devaluation (Figure 4.1), the decline in survival due to decreasing risk in the security games was larger. Therefore, the hypothesis model predicted a decline in survival after Box 5:5 in the trade-off games. While the negative trend observed in the trade-off games was weaker than expected from the hypothesis model, the average survival of balls did decrease as devaluation increased and security decreased. The dynamic games also support this negative trend in survival as devaluation increased and security decreased. Participants preferred to select from boxes with more red balls and fewer blue balls to avoid the risk posed by blue balls, even when red balls reduced in value from 50 cents to 20 cents. In the scavenger-hunt games, high numbers of stakes were 'killed' across all three devaluation levels, again suggesting that high levels of devaluation failed to protect commodities. Furthermore, in Section 1A of the survey, all pairwise comparisons that included Park 1 with $0 \%$ devaluation showed that this park was consistently the least preferred park. This was confirmed by Section 1B of the survey where Park 1 was ranked lowest more often than would be expected. Devaluation might be discouraging to hunters but is seldom their only consideration. In particular, risk rendered devaluation strategies less effective at protecting commodities.

Unlike devaluation, security as a sole measure can effectively reduce illegal hunting. While sometimes criticised, enforcing conservation laws is an important regulator of wildlife crime (Keane et al., 2008). In Zambia for example, higher resource allocation to enforcement efforts reduced the illegal hunting of elephants (Jachmann \& Billiouw, 1997). High levels of security were more effective at improving ball survival than high levels of devaluation. In the devaluation games, survival increased as devaluation increased. However, the decline in survival as security decreased in the security games was steeper and of greater magnitude. Risk, in the form of security, more effectively deterred hunting compared to devaluation of a similar magnitude. The behaviour of the guards in the dynamic games reflected this. By consistently placing high numbers of blue balls in their boxes, the guards demonstrated an understanding that risk is a much more effective deterrent than devaluation. The scavenger- 
hunt games also demonstrated the importance of security. The risk of laying a stake down was perceived as low, resulting in all worthless stakes that were found being laid down to be removed from the game. Whether this level of risk is consistent with that which real-world hunter's experience and perceive is debatable.

The risks associated with illegal hunting are often said to be low. This can be due to lenient penalties, low detection rates, and corruption (Bennett, 2015; Leader-Williams \& Milner-Gulland, 1993; Sollund, 2016). Enforcement of conservation laws through security is often described as inefficient, as shown by the growth of the IWT despite increased investment in security (Challender \& MacMillan, 2014). Additionally, when killing a devalued animal, the scene can be vacated immediately as the commodity is often not harvested. With no commodity having been harvested, there is also less chance of being apprehended after leaving the park. Historically, penalties have also been lenient. Some countries have introduced harsher penalties, such as longer prison sentences in South Africa (Milliken et al., 2012), and shoot-to-kill policies in Zimbabwe (Leader-Williams \& MilnerGulland, 1993). Nevertheless, it is suggested that many countries such as Kenya should increase penalties as a deterrent (Milliken et al., 2012). Many of the countries where illegal hunting is common also experience high levels of corruption (Bennett, 2015; Challender \& MacMillan, 2014). Corrupt officials overlook wildlife crime infringements, facilitate the introduction of contraband to legal markets (Bennett, 2015) and disclose sensitive information, such as the location of rhino (Taylor et al., 2014). For these reasons, the low level of risk that participants experienced when laying down a stake is arguably consistent with the real-world.

Failed attempts to substitute security with devaluation has been observed with dehorning in Africa. Many parks have dehorned rhino prior to security reductions or in response to poor security. When dehorning was attempted in Zimbabwe's Hwange National Park, nearly all rhino were subsequently killed (Lindsey \& Taylor, 2011). This may be because the Department of National Parks and Wild Life Management experienced budgetary reductions in the 12-18 months following dehorning which resulted in security lapses (Lindsey \& Taylor, 2011). Other Zimbabwean parks including Matobo National Park, Matusadona National Park, Chipinge Safari Area and Sinamatela also experienced significant losses of rhino following dehorning and in the absence of security (Lindsey \& Taylor, 2011). More recently, the Save Valley Conservancy dehorned six rhino in 2011, all of which were subsequently killed (Lindsey \& Taylor, 2011). 
Security has also been shown to be more effective than devaluation. The Mililangwe Wildlife Reserve has one of the most comprehensive security programmes in Zimbabwe (Du Toit, 2011). Rhino hunting rates are low and therefore dehorning is not necessary (Du Toit, 2011). In comparison, parks that have implemented devaluation to compensate for poor security have been unsuccessful. Three poorly protected reserves each experienced a 10-fold increase in rhino kills compared to Mililangwe Wildlife Reserve, despite on average $41 \%$ of these populations being dehorned (Du Toit, 2011). The Chiredzi River Conservancy, another reserve with poor security, lost 27 of its 29 rhino all of which had been dehorned (Du Toit, 2011). If hunter detection rates remain low and devaluation is more widely used, the killing of devalued animals could become a common occurrence. Devaluation should be accompanied by security if it is to provide any benefits, and security should be prioritised if resources are limited.

\subsubsection{Risk aversion vs. Risk tolerance}

Devaluation failed to protect commodities because the responses to devaluation are more variable than responses to security. People responded to devaluation differently. Some were risk averse and preferred to hunt in low risk areas despite reduced rewards. Where there is a trade-off between security and devaluation, the devalued population may still be hunted, even increasingly, especially if the devalued animals retain some value.

Risk aversion can be defined as "the tendency to prefer certain rewards over (often larger) rewards of uncertain probability" (Sheehy-Skeffington \& Rea, 2017, p. 46). In the games, this relates to claiming the value of a devalued red ball, rather than taking the risk of trying to find a yellow ball of higher value. Conversely, risk tolerance can be defined as "the tendency to prefer rewards of uncertain probability over (often smaller) rewards of certain probability" (Sheehy-Skeffington \& Rea, 2017, p. 46). In the games, this relates to returning or discarding a devalued red ball and taking the risk of trying to find a yellow ball of higher value. As devaluation increased in the trade-off games, the coefficient of variation for the survival of balls increased. There was greater variation in how participants responded to devaluation, compared to how participants responded to security. This divergence is also demonstrated by the lucky-dip post-game surveys. Those who received a reward (winners) adopted consistent strategies, compared to those who did not receive a reward (losers), showing differences in the way people played the game. In the online survey, respondents were divided on how they ranked Park 4 with the greatest level of devaluation (90\%). Park 4 
was frequently ranked first and fourth, with fewer second and third ranks. This was the only park that presented this polarised result. The divergence of behaviour in response to devaluation might occur because the risk imposed by devaluation is indirect. Devaluation aims to reduce the hunter's rewards, and therefore provide a disincentive to hunt (Büscher \& Ramutsindela, 2016; Ferreira, Hofmeyr, et al., 2014). Conversely, the risk imposed by security was direct and absolute, meaning everyone perceived and responded to it similarly.

\subsubsection{Changes in perceptions of risk and value}

Devaluation also failed to improve survival because the interaction between security and devaluation caused people's perceptions of risk and reward to change. The interaction between perceived risk and perceived value is a well-studied phenomenon. Proposed by Kahneman and Tversky (1979), prospect theory was developed in response to the critiques of expected utility theory and is promoted as a better explanation of decision making. It focuses on decision making between probability choices, more commonly known as a gamble (Kahneman \& Tversky, 1979). Expected utility theory looks at how people make optimal choices based on the assumption that people make decisions to maximise their utility (Myerson, 1991). Conversely, prospect theory claims that people's decision making is based on the potential magnitude of risks and rewards instead of the outcome, and explains behaviours that do not maximise utility (Kahneman \& Tversky, 1979). For example, consider a choice between $90 \%$ chance of $\$ 1,000$ and a $10 \%$ chance of $\$ 0$, or $100 \%$ chance of $\$ 890$. Using expected values, people should select the $90 \%$ chance of $\$ 1,000$ because the expected value, $\$ 900(\$ 1,000 \times 0.90=\$ 900)$, is higher than $\$ 890$. However, prospect theory explains why most people select the $100 \%$ chance of $\$ 890$ (Kahneman \& Tversky, 1979).

The presence of risk increased people's perceived relative value of devalued items. Participants behaved differently towards red balls in devaluation versus trade-off games. In the devaluation games, very few red balls were kept with the majority returned or discarded. However, in the trade-off games most red balls were kept and few were returned or discarded. Therefore, the increase in risk caused people to adopt more risk averse behaviours as they were more likely to keep a red ball and settle for its lower value. This could also be explained as risk causing the perceived relative value of red balls to increase. As a result, where devaluation does not render the commodity completely worthless, more hunters may kill animals that have been devalued to harvest the remaining valuable commodity. 
Paulsen et al. (2011) presented participants with two gamble choices with the same expected value. As the difference in risk between the two gambles increased, participants were more inclined to choose the low risk-reward option (Paulsen et al., 2011). Therefore, despite no difference in the expected values, risk aversion increased with increasing risk (Paulsen et al., 2012). Similar behavioural trends have been observed in law. Tor, GazalAyal, and Garcia (2010) hypothesised that the decision to accept a plea deal or go to trial is largely determined by the severity of the punishment and the probability of being found guilty. Using questionnaires, they found that as the risk of going to trial increased, participants were more inclined to exhibit risk averse behaviour by accepting a plea deal (Tor et al., 2010).

\subsubsection{Magnitude of devaluation}

The 'killing' of devalued items was more common when devalued items were worthless. This is associated with the concern about devaluation methods being unable to fully devalue commodities. The magnitude of devaluation can be defined as the proportion of the commodity's value that is removed. This will be an important consideration if new devaluation methods are developed. Devaluation methods currently developed (e.g. rhino dehorning and horn infusions) do not render the commodity worthless. Some valuable commodity always remains, and the horns regrow. Dehorning only removes $90 \%-93 \%$ of the horn (Kock \& Atkinson, 1993), and must be repeated every 1.3 years to account for regrowth and successfully discourage hunting (Milner-Gulland et al., 1992). The literature on devaluation suggests that partial devaluation is an issue, with the implication being that complete devaluation would be more effective (Büscher \& Ramutsindela, 2016; Ferreira, Hofmeyr, et al., 2014; Lindsey \& Taylor, 2011). My findings contradict this expectation. When red balls were worth 50 cents, few were discarded, and many were either kept or returned. However, when red balls were worthless, most were discarded and few were kept. Those participants who chose not to keep red balls were more likely to discard them if they were worthless. A similar result was observed in the scavenger-hunt games with all devalued stakes being laid down. Devalued stakes were always worthless and laying down a stake was comparable to with discarding a ball in the lucky-dip games.

There are several reasons why a hunter may kill a worthless animal. Some hunters will kill a devalued rhino in anger or spite of not finding a more valuable animal (Martin, 1994). Additionally, if you eliminate the devalued animal from the population, you remove the risk 
of tracking the same animal again in the future (Lindsey \& Taylor, 2011). It can be difficult to determine an animal's value from distance or in thick bush, so the animal is sometimes killed and the commodity's value later inspected in a safe location (Lindsey \& Taylor, 2011). Killing a worthless animal can also be a form of retribution against the guards or the park that is attempting to regulate illegal hunting (Lindsey \& Taylor, 2011).

This could suggest that if a hunter encounters a devalued animal, they are more likely to leave it alive if the devalued commodity retains some value. They are more likely to kill the animal if the devalued commodity is worthless. This is especially true if the perceived risk of detection having killed the animal is low (Leader-Williams \& Milner-Gulland, 1993; Sollund, 2016). This behaviour could be explained by several rationales. Firstly, people find it difficult to discard an item of value, even if that value is considered by them to be low. The desire to collect and accumulate goods has evolved in humans, as it increases the probability of survival in times of scarce resources (Grisham \& Barlow, 2005). In modern society, collective behaviours are a basic human instinct (Grisham \& Barlow, 2005). Therefore, even though a participant may have chosen not to take the risk of keeping a devalued item, they also did not want to throw away an item of value. Secondly, participants could be attempting to control the proportion of balls in the boxes or the outcomes of other participants. Some participants reported that they returned red balls because even though they did not want the value, another participant may. This implies a cooperation motivation. Others reported that returning a red ball would disadvantage others because they would be more likely to select a red ball as opposed to a full-value yellow ball. This implies a competition motivation. Therefore, partial devaluation may be more successful than complete devaluation if the remaining value is low enough to be undesirable but high enough to not be considered worthless. Finding this balance will be difficult and context-specific but could assist with the development of successful devaluation strategies.

\subsubsection{Devaluation did not demotivate participants}

Finally, devaluation failed to demotivate participants. Following the scavenger-hunt games, there was no difference in how participants scored their motivation across the three devaluation levels. Additionally, there was no difference in the average number of steps taken per participant pair across devaluation levels. While some participants reported higher motivation for fewer high-value items, others reported being more motivated by many lowvalue items. Additionally, some stated that their motivation did not differ between the 
devaluation levels because the total value available (\$250) was constant across all games. Similar results were obtained during the pilot studies when measures of motivation included time spent searching and the proportion or invited participants that participated (Figure 2.2 and Figure 2.3). When interpreting these results, one must consider that only the level of devaluation was manipulated between scavenger-hunt games. Because the level of risk remained constant, one could conclude that devaluation failed to demotivate participants.

\subsection{Impacts across multi-park systems}

As well as showing that devaluation failed, the games demonstrate how the strategies to prevent illegal hunting in one park could impact hunting pressure at surrounding parks. The success of any hunting prevention strategy will depend on whether the park is isolated or one of many parks available to hunters. If the park is geographically isolated, hunting prevention strategies might be less effective because hunters have less opportunity to shift their hunting activities to a more desirable park. While risk averse hunters may stop hunting altogether because the risks have increased too substantially, risk tolerant hunters will continue hunting.

However, if hunting prevention strategies are altered or applied at a park that is not geographically isolated, these actions could impact hunting pressure at neighbouring parks. Applying or increasing security or devaluation at a park may cause illegal hunters to shift their hunting efforts to other nearby parks (Lindsey \& Taylor, 2011). In South Africa, most dehorning is conducted by private parks (Lindsey \& Taylor, 2011). Apart from provincial parks in the Mpumalanga Province, South Africa National Parks (SANParks) and provincial parks do not dehorn rhino (Taylor et al., 2014). As private game reserves are relatively profitable and small (Langholz \& Lassoie, 2001), they could be in a better financial position to dehorn rhino without having to reduce security. Neighbouring national or provincial parks may be unable to afford to devalue their larger and more difficult to capture populations. Therefore, as dehorning increases in private parks, the illegal hunting pressure could shift to neighbouring parks (Lindsey \& Taylor, 2011; Milliken et al., 2012).

The lucky-dip games were designed to study this phenomenon because each box represented a different park. In the devaluation and security games, participants targeted boxes with lower levels of risk and devaluation. In the trade-off games, most selections were made at the central box (Box 5:5) where the number of red, yellow and blue balls was equal. The number of selections tailed off either side of Box 5:5 in a bell-curve shape towards the 
security and devaluation extremes. This may be because people were less willing to select from either extreme (security or devaluation) and instead selected from the boxes with moderate levels of risk and reward. Another explanation is that the majority of participants were risk neutral, meaning they are indifferent to extreme levels of risk and reward (Concina, 2014). If that were true, it would contradict previous research which has concluded that risk aversion is the most common behavioural trait, followed by risk neutrality, with risk tolerance being least common (Bergman, 2004; Concina, 2014; Dohmen et al., 2011; Holt \& Laury, 2002). Risk aversion potentially increases as the rewards increase (Concina, 2014). This would explain why risk averse behaviour was less common than expected in these games as the rewards were relatively low. However, the Holt and Laury (2002) study also utilised low rewards, with the maximum gain being less than $\$ 4$, and found that $66 \%$ of their participants were risk averse, $25 \%$ risk neutral and $8 \%$ risk tolerant.

Which box participants chose to select balls from also influenced the distribution of blue ball selections. It is important to remember when interpreting these results that all the boxes contained different numbers and proportions of blue balls (Figure 4.4). It was hypothesised that the highest number of blue balls would be selected at Box 10:0, where the total number and proportion of blue balls was highest. Surprisingly, the number of blue balls selected did not differ across the first five boxes. Because more selections were made at the central boxes (Boxes 7:3, 5:5 and 3:7), the number of blue balls selected at these boxes was higher than expected. This result was confirmed in the dynamic games, where the number of blue balls selected from a box was only weakly correlated with the number of blue balls in the box $(\mathrm{R}=0.243)$. A stronger correlation was expected.

These results suggest that most people are deterred by extreme levels of risk and reward, and prefer a moderate risk-reward trade-off. Therefore, it will not necessarily be the park with the highest security level that will succeed in apprehending the most illegal hunters. The number of illegal hunters apprehended will be determined by a combination of security efficiency and hunting pressure. Increasing the efficacy of security could force hunters to select other surrounding parks, resulting in more apprehensions there. Therefore, protecting a meta-population of wildlife may be enhanced by coordinating the levels of security between neighbouring parks. This result also confirms that security is effective at deterring illegal hunters without necessarily resulting in a higher number of apprehensions. 


\subsection{Socio-economic considerations}

Participants responded differently to devaluation across the five sites where lucky-dip games were conducted. In Petone and Upper Hutt, many devalued red balls were kept. Conversely, in Aro Valley, Karori and Seatoun, fewer red balls were kept, and many were discarded. This can potentially be explained by the different socio-economic characteristics of the communities in which the games were conducted. Most red balls were discarded in Karori and Seatoun where residents are of higher socio-economic status. In Upper Hutt and Petone where more red balls were kept, residents are of a lower socio-economic status. The results in Aro Valley were more similar to those in Karori and Seatoun, although Aro Valley's socioeconomic status is less clear. While a high deprivation index and low household income correspond with the lower socio-economic sites (Petone and Upper Hutt), labour force participation and tertiary education correspond with the higher socio-economic sites (Karori and Seatoun) (Figure 4.8). In New Zealand, socio-economic status is highly correlated with tertiary education (The New Zealand Ministry of Education, 2006). Aro Valley is near Victoria University of Wellington, and a high percentage of the residents are expected to be university students. This could explain why the response to red balls in Aro Valley was more in line with the other higher socio-economic sites.

This study was not designed to evaluate the influence of socio-economic influences on behaviour. If it had been, a greater number of games and participants would have been required to achieve statistical power so that those measures could be made across individuals, not sites. Nevertheless, the trends across sites in how the game was played appears to be consistent with previous research. It is often assumed that those of lower socioeconomic status engage in greater risk-taking behaviour (Sheehy-Skeffington \& Rea, 2017). However, the results support previous research that those of lower socio-economic status are more riskaverse than those of higher socio-economic status. One study that demonstrates this conducted an economic game that involved blowing up a balloon as a risk measure (Amir, Jordan, \& Rand, 2018). The larger the balloon got the more the participant was paid, but if the balloon popped, the participant received nothing (Amir et al., 2018). A second game involved hypothetically asking participants whether they would prefer a $50 \%$ chance of receiving $\$ 800$, or a guaranteed lesser value of between $\$ 100$ and $\$ 700$ (Amir et al., 2018). Those whose childhood socio-economic status was lower were more risk averse (Amir et al., 2018). Another study showed that there was a correlation between student socio-economic status and the choice of a career with high labour income risk (Caner \& Okten, 2010). Labour 
income risk is defined as the unpredictability of income (Caner \& Okten, 2010). Students from lower socio-economic backgrounds were less likely to select higher income risk careers (Caner \& Okten, 2010).

This result could indicate that the origins of the illegal hunter may dictate how they respond to security and devaluation strategies. An illegal hunter that hunts because they do not have an income or have local debt might be more risk averse and favour hunting a devalued population if risks are lower. Alternatively, an outsider who is attracted by highvalue might be more risk tolerant and favour hunting more protected populations in search of high-value animals. The success of devaluation strategies could be influenced by whether hunting is conducted by poverty driven local villagers, or greed driven criminal syndicates. Assessing this hypothesis further in future research would be a worthwhile and useful endeavour.

\subsection{Effectiveness of games at studying human behaviour}

This research has been a successful proof of concept for using games to research human behaviour and test hypotheses around wildlife hunting and trade. Firstly, the games have confirmed many of the conclusions and expectations that theoretical studies and anecdotal evidence have provided. The results suggest that devaluation may not succeed if implemented individually, and that security is likely to be a more consistent and effective deterrent of illegal hunting. The results demonstrate how the actions of one park will influence hunting at surrounding parks, and are consistent with previous research that people from lower socioeconomic backgrounds are more risk averse than people from higher socio-economic backgrounds.

The games also uncovered behaviours that previous research had been unable to examine. Human behavioural responses to devaluation are divergent. While devaluation successfully discouraged some participants, it also encouraged others. Risk tolerant hunters are less likely to be discouraged by the higher risks associated with higher potential rewards. Conversely, risk averse hunters, which arguably comprise the majority, are encouraged by lower risks despite associated lower rewards. In addition, the literature suggests that rendering the commodity worthless would be necessary to ensure the success of devaluation strategies. However, these results suggest that partial devaluation of the commodity may 
benefit survival. Hunters who are not willing to harvest a partially devalued commodity are less likely to kill a devalued animal if it retains some value.

Finally, the rationales that participants provided for their behaviour in the post-game surveys aligned with the rationales provided by real-world illegal hunters. In the scavengerhunt games, all devalued items that were found by participants were removed from their standing position and laid on the ground to signify that they were removed from the game. This action represented killing a devalued animal, even though there is no valuable commodity to be harvested. There are several reasons why a hunter may kill a worthless animal including anger or spite, to prevent finding the same animal again, to remove and later assess the animals value in a safer location, or as a retributive action against guards (Lindsey $\&$ Taylor, 2011; Martin, 1994). Most of these rationales were reported in the scavenger-hunt post-game surveys. Of the 31 respondents who laid down a worthless stake, 10 (32\%) indicated that they did so to prevent finding it again in the future. In the pilot studies, some respondents stated that they laid down a worthless stake in anger, and some stated that they laid down a worthless stake so that the guards would not be rewarded for its survival.

The fact that some participants provided similar rationales for their behaviours as illegal hunters indicates that games were an effective method of modelling human behavioural responses to devaluation. Although few participants expressed these rationales, it does still demonstrate the potential effectiveness of utilising games to study human behaviour. These games are a starting point. With greater resources, it could be possible to develop games that are more representative of reality, and that result in more participants exhibiting behaviours and rationales consistent with those of illegal hunters.

\subsubsection{Enhancements}

There are several ways in which this research could be enhanced if it were to be repeated or replicated. Many of the enhancements suggested below were known from the pilot studies. However, they were not included in this research due to financial or time limitations, and to achieve simplicity. Each scavenger-hunt game cost $\$ 250$ while lucky-dip games cost $\$ 20$ on average. Additional costs included equipment and registration fees for the public events. The research also had to be completed in the one-year allotted for a Masters thesis. These limitations meant that conducting more games was not feasible, which led to the decision to 
keep the games relatively simple. More complex games are possible and suggested if resources allow.

The lucky-dip games assumed a 1:1 financial trade-off between security and devaluation. While this assumption was made to achieve simplicity, adopting a more realistic financial trade-off would provide interesting and potentially more reliable results. The exact financial trade-off between security and devaluation is unknown. The cost of security differs between areas depending on factors such as size, terrain, isolation, infrastructure and access to employees (Rosen \& Smith, 2010). Likewise, certain populations can be more expensive to devalue due to factors such as the size and distribution of the population, the terrain and access to equipment (e.g. helicopters) and expertise (e.g. veterinary) (Ferreira, Hofmeyr, et al., 2014). Implementing effective security to protect wildlife is expensive, especially for high-value species such as rhino (Ferreira, Pfab, \& Knight, 2014). In the 1980's every $\$ 20,000$ spent on enforcement in Zambia resulted in only \$1 of additional cost to illegal hunters (Milner-Gulland, 1999). Whilst devaluation also requires significant financial investment, it is suggested to be more cost effective than security (Milner-Gulland, 1999). I advise developing games where security requires a greater investment than devaluation. This could be achieved by halving the proportion of blue balls (security) in each box but retaining the same proportion of red balls (devaluation). This would result in a security-devaluation trade-off of 2:1. One would expect results more in favour of devaluation, because the risk posed by blue balls would be less, and participants would be more inclined to select from the security boxes. Alternatively, a more flexible experimental design with multiple trade-off ratios could be beneficial since the true trade-off ration is unknown and likely to differ. Devaluation at a park could increase illegal hunting at surrounding parks with poor security, because hunters discouraged by devaluation will shift to hunting in these parks.

It would also be interesting to manipulate risk at the buyer's box. The risk at the buyer's box represented the risk of delivering and selling the commodity to a buyer (i.e., trading). Increasing the proportion of blue balls here would represent an increase in the efficiency of policing outside of protected areas, such as the identification and arrest of IWT participants. Little is known about how manipulating risk at this level of the supply chain will affect the system. Current knowledge focusses on enforcement at the hunting level of the supply chain through the use of rangers and security. However, increased policing outside of parks rather than security inside parks could be easier and more effective. The distribution of illegal wildlife commodities will experience bottlenecks in villages and at border crossings 
and ports. Focussed policing at these bottlenecks, as is done to prevent the distribution of illegal drugs by targeting border crossings, could be more effective (Dell, 2015; INTERPOL, 2015).

Games could be designed to test the effectiveness of enforcement at different levels of the supply chain. If the proportion of blue balls at the buyer's box was increased, participants would be less inclined to keep and claim the value of a devalued red ball. This is because the risk of claiming a ball's value would be higher, and only worthwhile if the ball was relatively valuable. In the real-world this would translate to hunters deciding not to harvest a partially devalued commodity. Increasing the efficiency of policing outside of parks would probably have little effect on whether the hunter decides to kill or spare a devalued animal. That decision is more influenced by the magnitude of devaluation, the proportion of the population devalued and the risk a hunter faces of being detected in the protected area.

It would be beneficial to conduct scavenger-hunt games where the devalued items have some value rather than being worthless. Unlike the lucky-dip games, all the devalued stakes in the scavenger-hunt games were worthless. This decision was made to simplify the game because the game could not be highly replicated. This most likely contributed to all the devalued stakes that were found being laid on the ground and removed from the game. However, current devaluation methods do not render the commodity completely worthless, and the lucky-dip games suggest that partial devaluation might be more beneficial. Therefore, conducting scavenger-hunt games with devalued items worth a proportion of the full-value commodity would be useful. Based on the results of the lucky-dip games, more devalued items would be expected to be left standing if they retained some value.

It would also be beneficial to manipulate the risk associated with laying down a stake. This could be achieved by increasing the number of guard participants or increasing the handling time or risk of laying a stake down. For example, a loud sound could play when a stake is manipulated (simulating a gunshot) to inform the guards of the "kill" and the location. The games suggest that the risk of laying a stake down was low because all the devalued stakes that were found were laid down. While this level of risk may be representative of the real-world, the games and literature suggest that risk is an effective and consistent regulator of human behaviour. It would be beneficial to examine how different levels of risk in the scavenger-hunt game influence participant responses to devalued items. 
Conducting more variations of the scavenger-hunt game, as was done with the luckydip game, would be beneficial. Conducting many variations of the lucky-dip game was possible because the games were quick to conduct. Due to participant recruitment challenges and the length of the scavenger-hunt games, conducting as many variations and replicates was not feasible. Nevertheless, conducting more scavenger-hunt games would be beneficial because they provide useful information about the complex and nuanced human behaviours that are not exhibited in the lucky-dip games.

All scavenger-hunt participants completed a post-game survey. The survey responses from the pilot studies were more complex than the responses received for the final applications of the games. I believe this was primarily due to the longer length of the pilot study games. Whilst the games were only 2.5 hours long, the games used in the two pilot studies were conducted over two and three days respectively. The pilot study respondents reported more complex behaviours, interactions, cooperation deals and competitive behaviours. While some participants reported similar behaviours following the final application of the games, these responses were less complex and less frequent. The longer games may have given participants more time to devise complex behavioural and interactive strategies (cooperative and competitive) that are more realistic of the real-world. Cooperation deals were common in both the pilot studies and the games. However, bribery and competition between hunters, and retributive actions by hunters towards guards, were all exhibited in the pilot studies but were not exhibited in the games. These behaviours are exhibited by illegal hunters (Bennett, 2015; Lindsey \& Taylor, 2011).

Unfortunately, conducting more scavenger-hunt games, and longer games would make it more difficult to recruit participants. Recruitment for the pilot studies was easier because they were conducted with university students on campus as part of an undergraduate course. The games were conducted with a more general population and were therefore shortened to facilitate recruitment. Despite this, it was still difficult to recruit organisations and participants, resulting in only four scavenger-hunt games being conducted with a total of 48 participants. Nevertheless, conducting longer scavenger-hunt games would provide results that are more representative of the real-world behaviours, but would require more time, personnel and investment of resources. 


\subsection{Real-world application of the research}

Applying the results of this research to the real-world would first require testing the games with a more contextually relevant population. This could be achieved by taking the games to local communities in regions such as Africa or south-east Asia where illegal hunting is prevalent. The unique socio-economic and cultural characteristics of these communities could therefore be considered. Illegal hunters are typically from low socioeconomic backgrounds (Duffy et al., 2016) and have lower socio-economic status than the participants that played the games. In these games, lower socioeconomic sites tended to exhibit more risk averse behaviour. Participants from local communities in Africa or south-east Asia would presumably be more risk averse and align more similarly with the lower socioeconomic sites (Petone and Upper Hutt) rather than the higher socioeconomic sites (Karori and Seatoun). This might mean that they target devalued populations, and more devalued animals could be killed.

The literature on risk aversion in African communities is divided. Brick, Visser, and Burns (2012) assessed risk aversion in South African fishing communities, with regards to illegal fishing. While socio-economic status was not considered, no correlation was discovered between risk aversion and wealth, an important socioeconomic variable (Brick et al., 2012). Conversely, Yesuf and Bluffstone (2009) examined poverty and risk aversion in Ethiopian communities. They used a number of wealth indicators (livestock, land size and cash availability) and found that increased wealth was correlated with greater risk tolerance (Yesuf \& Bluffstone, 2009). A similar result was obtained by a risk aversion study in Zambia (Wik et al., 2004). These results confirm the decreasing absolute risk aversion (DARA) hypothesis which explains that increased wealth (and by extension socioeconomic status) results in greater risk tolerance (Wik et al., 2004). Therefore, risk aversion increases with declines in wealth and socio-economic status.

After testing the games in more contextually relevant communities, the results would need to be applied to the real-world. This would need to be done through adaptive management. Adaptive management is a decision making process used in conditions of uncertainty that allows for the outcomes of an implemented management initiative to be continually monitored and evaluated as a guide to future decisions (McCarthy \& Possingham, 2007). This allows for the early detection of unanticipated outcomes, and for management initiatives to be adapted accordingly (McCarthy \& Possingham, 2007). An adaptive 
management approach would be necessary for two reasons. Firstly, although games were an effective method of studying human behaviour, they are a model of human behaviour. As with all models there will be unpredictable differences between the model and reality (Redpath et al., 2018). This is especially true of these games as they have been conducted with participants disconnected from the IWT system (Redpath et al., 2018). This is the reason for first testing the games with a contextually relevant population. An adaptive management approach will enable improvement of the management initiative in response to any unpredicted outcomes. Secondly, the outcome of any management initiative, such as devaluation, will be context dependent. The outcome will differ between species and protected areas based on their unique characteristics. An adaptive management approach could enable the management initiative to be adapted to each situation.

Lindsey and Taylor (2011) provide a framework for use as a decision-making tool on when and how to implement dehorning. Based on this framework, other research and the results of this study, I offer the following recommendation. If threatened by illegal hunting, small populations of rhino should be completely devalued, so long as effective security can be maintained following devaluation. Maximising both security and devaluation will be the most successful strategy but is often unachievable due to financial or logistical limitations. If implementing devaluation results in decreased resources for security, then security should be prioritised over devaluation. However, some protected areas may have increased the magnitude of their security programs beyond the point where significant additional benefits are received. In these situations, it may be acceptable to reduce security to the point where the benefits are maintained and use the recovered revenue to implement devaluation.

For moderate and large populations of rhino where devaluing the entire population is not possible, devaluation should be implemented to a level that does not require a reduction in security. In the trade-off lucky-dip games, Box 9:1 was the most successful because survival was highest, the number of selections was lowest (highest level of deterrence) and the number of selections per blue ball selected (catch efficiency) was highest. Although, the outcomes for Box 9:1 were not statistically different from adjacent boxes, this may indicate that limited devaluation has an effect when security can be maintained. Devaluing a small proportion (e.g. 10\%) of moderate to large rhino populations may provide some benefit over only using security. 


\subsection{Non-economic concerns with devaluation}

Besides economic considerations, there are other concerns associated with devaluation strategies. Tranquilising an animal is a difficult and risky procedure (Lindsey \& Taylor, 2011). When dehorning of rhino was first attempted in the late 1980's, mortality rates were high at 14\% (Lindsey \& Taylor, 2011). In 1991, 7\% of 141 white rhino tranquilised in Zimbabwe died (Kock et al., 1995). Although the procedure has improved, and deaths are now rare, tranquilisation mortality is a risk that needs to be considered when devaluing any species. There are also concerns that the tranquilisation reduces female rhino fertility. Alibhai et al. (2001) found that an intensive immobilisation programme in Zimbabwe had a negative impact on female rhino fertility. The risks of tranquilising any animal need to be considered, especially if a safe tranquilisation protocol has not been developed for the species (Kock et al., 1995; Lindsey \& Taylor, 2011).

There are also concerns that dehorning results in increased calf mortality as the mother is less equipped to protect her calf from predators (J. Berger \& Cunningham, 1994). This research has been questioned due to small sample sizes (Lindsey \& Taylor, 2011). Nevertheless, the negative consequences that devaluation could have on intra- and interspecific interactions should be considered when devaluing any species.

The removal of any valuable commodity from an animal, such as dehorning rhino, likely results in the storage and stockpiling of the valuable commodities. Criminals have attempted to raid stockpiles of commodities such as rhino horn and ivory (Milliken et al., 2012). While SANParks have secure storage facilities, such as the Kruger National Park storage facility at Skukuza Camp, private parks often do not have this infrastructure (Milliken et al., 2012). Many private parks store rhino horns in bank vaults (Lindsey \& Taylor, 2011). However, some banks refuse to accept these commodities as they carry extreme security risks. Private parks then incur further cost by having to utilise private security companies (Lindsey \& Taylor, 2011). If valuable wildlife commodities are removed from any species, consideration must be given to the security, storage and potentially the destruction of the commodities.

Finally, devaluation strategies could negatively impact tourism. With rhino being one of the Big 5 game species, it was suggested that tourists would be less inclined to visit a park that had dehorned rhino (Lindsey \& Taylor, 2011). While some tourists objected to dehorning when it was first attempted, some suggested that dehorning could benefit tourism as it 
demonstrates that the park is actively attempting to prevent illegal hunting (Lindsey \& Taylor, 2011). Nevertheless, when applying devaluation, decision makers should consider public opinion of the proposed method, and whether their decisions will negatively impact tourism. Many parks rely on tourism as a main source of revenue to implement and maintain hunting prevention strategies such as security and devaluation (Langholz, 1996).

\subsection{Conclusion}

For several decades, devaluation has been promoted as a way to reduce the reward for illegal hunters and discourage illegal hunting. However, devaluation strategies may not be the silver bullet solution that proponents suggest. This research shows that devaluation can fail because people respond to devaluation differently, and security is a more effective and consistent regulator of human behaviour. Contrary to expectation, rendering a commodity worthless may result in more killings than if the commodities retain some value. Furthermore, decision makers should be aware that the actions of one park might impact the hunting pressure and success of hunting prevention strategies at neighbouring parks. While devaluation may be effective against some illegal hunters, other hunters may be incentivised to hunt devalued populations, especially if security has been reduced as a result. This does not imply that devaluation strategies are not useful. Devaluation may provide additional protection when it is not possible to improve security or where additional security would provide negligible benefits.

A multifaceted approach will be required to resolve the IWT (Challender, $\mathrm{Wu}$, et al., 2014). This will require a combination of security, devaluation, and technology, as well as long-term strategies such as demand reduction, poverty alleviation and community involvement. To achieve a multifaceted strategy, the field of conservation biology should welcome and facilitate interdisciplinary collaboration with fields such as the social sciences and psychology. The use of games contributes to this multifaceted and interdisciplinary approach. Games can provide an effective method of researching human behaviour and modelling complex and difficult to study real-world systems. Many other difficult-to-study disciplines, such as criminology, could benefit from greater use of similar methods to overcome research barriers. I encourage more researchers and disciplines to embrace the utility of games as a research tool. 



\section{References}

Alibhai, S. K., Jewell, Z. C., \& Towindo, S. S. (2001). Effects of immobilization on fertility in female black rhino (Diceros bicornis). Journal of Zoology, 253(3), 333-345.

Almeida, J. E., Rossetti, R. J. F., Jacob, J. T. P. N., Faria, B. M., \& Leça Coelho, A. (2017). Serious games for the human behaviour analysis in emergency evacuation scenarios. Cluster Computing, 20(1), 707-720.

Amir, D., Jordan, M. R., \& Rand, D. G. (2018). An uncertainty management perspective on long-run impacts of adversity: The influence of childhood socioeconomic status on risk, time, and social preferences. Journal of Experimental Social Psychology, 79, 217-226.

Angelici, F. M. (2016). Problematic Wildlife at the Beginning of the Twenty-First Century: Introduction. In F. M. Angelici (Ed.), Problematic Wildlife: A Cross-Disciplinary Approach (pp. 3-18). Switzerland: Springer.

Annecke, W., \& Masubelele, M. (2016). A Review of the Impact of Militarisation: The Case of Rhino Poaching in Kruger National Park, South Africa. Conservation and Society, 14(3), 195-204.

Ayling, J. (2013). What sustains wildlife crime? Rhino horn trading and the resilience of criminal networks. Journal of International Wildlife Law \& Policy, 16, 57-80.

Baker, J. E. (1997). Development of a model system for touristic hunting revenue collection and allocation. Tourism Management, 18(5), 273-286.

Bates, D., Maechler, M., Bolker, B., \& Walker, S. (2015). Fitting Linear Mixed-Effects Models Using lme4. Journal of Statistical Software, 67(1), 1-48.

Bennett, E. L. (2015). Legal ivory trade in a corrupt world and its impact on African elephant populations. Conservation Biology, 29(1), 54-60. 
Berger, J., \& Cunningham, C. (1994). Phenotypic Alterations, Evolutionarily Significant Structures, and Rhino Conservation. Conservation Biology, 8(3), 833-840.

Berger, J. O. (1985). Statistical decision theory and Bayesian analysis (2 ed.). New York, NY: Springer-Verlag.

Bergman, M. (2004). Examining risk attitudes. Complexity, 9(5), 25-30.

Besanko, D., \& Braeutigam, R. R. (2005). Microeconomics (2 ed.). Hoboken, NJ: John Wiley $\&$ Sons.

Biggs, D., Courchamp, F., Martin, R., \& Possingham, H. P. (2013). Legal trade of Africa's rhino horns. Science, 339(6123), 1038-1039.

Boy, S. C., Raubenheimer, E. J., Marais, J., \& Steenkamp, G. (2015). White rhinoceros Ceratotherium simum horn development and structure: a deceptive optical illusion. Journal of Zoology, 296(3), 161-166.

Bozdogan, H. (1987). Model selection and Akaike's Information Criterion (AIC): The general theory and its analytical extensions. Psychometrika, 52(3), 345-370.

Brashares, J. S., Arcese, P., Sam, M. K., Coppolillo, P. B., Sinclair, A. R. E., \& Balmford, A. (2004). Bushmeat Hunting, Wildlife Declines, and Fish Supply in West Africa. Science, 306(5699), 1180-1183.

Brick, K., Visser, M., \& Burns, J. (2012). Risk Aversion: Experimental Evidence from South African Fishing Communities. American Journal of Agricultural Economics, 94(1), 133-152.

Burnham, K. P., \& Anderson, D. R. (2002). Model selection and multimodel inference: $a$ practical information-theoretic approach. New York, NY: Springer-Verlag.

Büscher, B., \& Ramutsindela, M. (2016). Green violence: Rhino poaching and the war to save Southern Africa's peace parks. African Affairs, 115(458), 1-22. 
Caner, A., \& Okten, C. (2010). Risk and career choice: Evidence from Turkey. Economics of Education Review, 29(6), 1060-1075.

Cartwright, E. (2011). Behavioral economics. New York, NY: Routledge.

Challender, D., \& MacMillan, D. C. (2014). Poaching is more than an enforcement problem. Conservation Letters, 7(5), 484-494.

Challender, D., Waterman, C., \& Baillie, J. E. (2014). Scaling up pangolin conservation. Retrieved from IUCN SSC Pangolin Specialist Group: http://cmsdata.iucn.org/downloads/scaling_up_pangolin_conservation_280714_v4_1. pdf

Challender, D., Wu, S. B., Nijman, V., \& MacMillan, D. C. (2014). Changing behavior to tackle the wildlife trade. Frontiers in Ecology and the Environment, 12(4), 203-203.

Chesney, T., Chuah, S.-H., \& Hoffmann, R. (2009). Virtual world experimentation: An exploratory study. Journal of Economic Behavior \& Organization, 72(1), 618-635.

Clarke, K. A., \& Primo, D. M. (2012). A model discipline: Political science and the logic of representations. New York, NY: Oxford University Press.

Colyvan, M., Justus, J., \& Regan, H. M. (2011). The conservation game. Biological Conservation, 144(4), 1246-1253.

Concina, L. (2014). Risk attitude \& economics (Number 2014-01). Retrieved from Toulouse, France: Foundation for an industrial safety culture: Foundation for an Industrial Safety Culture: https://www.foncsi.org/fr/publications/collections/regards/riskattitude-and-economics/Viewpoint-risk-attitude-economics.pdf

Cooney, R., Roe, D., Dublin, H., Phelps, J., Wilkie, D., Keane, A., . . Biggs, D. (2017). From Poachers to Protectors: Engaging Local Communities in Solutions to Illegal Wildlife Trade. Conservation Letters, 10(3), 367-374. 
Crookes, D. J. (2016). Trading on extinction: An open-access deterrence model for the South African abalone fishery. South African Journal of Science, 112(3-4), 1-9.

Crookes, D. J. (2017). Does a reduction in the price of rhino horn prevent poaching? Journal for Nature Conservation, 39, 73-82.

Crosta, A., \& Sutherland, K. (2016). The white gold of jihad: The 2010-2012 groundbreaking investigation into al-Shabaab's link to ivory trafficking in Eastern Africa. Retrieved from Los Angeles, CA: https://elephantleague.org/wpcontent/uploads/2016/02/Report-Ivory-al-Shabaab-Oct2016.pdf

Dang Vu, H. N., \& Nielsen, M. R. (2018). Understanding utilitarian and hedonic values determining the demand for rhino horn in Vietnam. Human Dimensions of Wildlife, $23(5), 417-432$.

Dell, M. (2015). Trafficking Networks and the Mexican Drug War. American Economic Review, 105(6), 1738-1779.

Dohmen, T., Falk, A., Huffman, D., Sunde, U., Schupp, J., \& Wagner, G. G. (2011). Individual Risk Attitudes: Measurement, Determinants, and Behavioral Consequences. Journal of the European Economic Association, 9(3), 522-550.

Dorward, L. J., Mittermeier, J. C., Sandbrook, C., \& Spooner, F. (2017). Pokémon go: benefits, costs, and lessons for the conservation movement. Conservation Letters, 10(1), 160-165.

du Toit, R. (2011). Zimbabwe lowveld: dehorning experience. Paper presented at the Proceedings of a workshop assessing legal trade in rhino horn as a tool in combating poaching as well as a detailed assessment of the efficacy of dehorning as a deterrent to poaching, Johannesburg, South Africa.

du Toit, R., \& Anderson, N. (2013). Dehorning rhinos. Wildlife Ranching, 2013 Autumn, 8285. 
Dudley, N., Stolton, S., \& Elliott, W. (2013). Wildlife crime poses unique challenges to protected areas. Parks, 19(1), 7-12.

Duffy, R. (2014). Waging a war to save biodiversity: the rise of militarized conservation. International Affairs, 90(4), 819-834.

Duffy, R., St John, F. A. V., Büscher, B., \& Brockington, D. (2016). Toward a new understanding of the links between poverty and illegal wildlife hunting. Conservation Biology, 30(1), 14-22.

Dutton, A. J., Hepburn, C., \& Macdonald, D. W. (2011). A Stated Preference Investigation into the Chinese Demand for Farmed vs. Wild Bear Bile. PloS one, 6(7), e21243.

Ellis, R. (2013). Tiger bone \& rhino horn: the destruction of wildlife for traditional Chinese medicine. Washington: Island Press.

Emslie, R. H. (2004). Rhino population sizes and trends. Pachyderm, 37, 107-110.

Emslie, R. H. (2012a). Ceratotherium simum. The IUCN Red List of Threatened Species 2012, e.T4185A16980466.

Emslie, R. H. (2012b). Diceros bicornis. The IUCN Red List of Threatened Species 2012, e.T6557A16980917.

Emslie, R. H., \& Brooks, M. (1999). African Rhino. Status Survey and Conservation Action Plan. Retrieved from IUCN/SSC African Rhino Specialist Group: https://portals.iucn.org/library/sites/library/files/documents/1999-049.pdf

Emslie, R. H., Milledge, S., Brooks, M., van Strien, N. J., \& Dublin, H. T. (2007). African and Asian Rhinoceroses - Status, Conservation and Trade. Retrieved from http://www.rhinoresourcecenter.com/pdf_files/118/1181374230.pdf

Emslie, R. H., Milliken, T., \& Talukdar, B. (2013). African and Asian Rhinoceroses - Status, Conservation and Trade. Retrieved from https://www.rhinoalive.com/wpcontent/uploads/2016/06/African-and-Asian-Rhino-Richard-Emslie.pdf 
Emslie, R. H., Milliken, T., Talukdar, B., Ellis, S., Adcock, K., \& Knight, M. H. (2016). African and Asian Rhinoceroses - Status, Conservation and Trade. Retrieved from http://www.rhinoresourcecenter.com/pdf_files/147/1470753643.pdf

Feng, Y., Siu, K., Wang, N., Ng, K.-M., Tsao, S.-W., Nagamatsu, T., \& Tong, Y. (2009). Bear bile: dilemma of traditional medicinal use and animal protection. Journal of Ethnobiology and Ethnomedicine, 5(2), 1-9.

Ferreira, S., Hofmeyr, M., Pienaar, D., \& Cooper, D. (2014). Chemical horn infusions: a poaching deterrent or an unnecessary deception? Pachyderm, 55, 54-61.

Ferreira, S., Pfab, M., \& Knight, M. (2014). Management strategies to curb rhino poaching: Alternative options using a cost-benefit approach. South African Journal of Science, 110(5-6), 01-08.

Fletcher, R. (2017). Gaming conservation: Nature 2.0 confronts nature-deficit disorder. Geoforum, 79, 153-162.

Foose, T. J., \& van Strien, N. (1997). Asian Rhinos - Status Survey and Conservation Action Plan. Retrieved from IUCN/SSC Asian Rhino Specialist Group: https://portals.iucn.org/library/sites/library/files/documents/1997-005.pdf

Game [Def. 1]. (n.d.). In Collins English Dictionary Online. Retrieved from https://www.collinsdictionary.com/dictionary/english/game

Garris, R., Ahlers, R., \& Driskell, J. E. (2002). Games, Motivation, and Learning: A Research and Practice Model. Simulation \& Gaming, 33(4), 441-467.

Gibbens, S. (2018, 31 March). Last Male Northern White Rhino Sudan Remembered in Ceremony. National Geographic. Retrieved from https://news.nationalgeographic.com/2018/03/sudan-last-male-northern-white-rhinoextinct-spd/ 
Gibson, C. C., \& Marks, S. A. (1995). Transforming rural hunters into conservationists: An assessment of community-based wildlife management programs in Africa. World Development, 23(6), 941-957.

Glynatsi, N. E., Knight, V., \& Lee, T. E. (2018). An Evolutionary Game Theoretic Model of Rhino Horn Devaluation. Ecological Modelling, 389, 33-40.

Griffin, E. (2007). Blood sport: hunting in Britain since 1066: Yale University Press.

Grisham, J. R., \& Barlow, D. H. (2005). Compulsive Hoarding: Current Research and Theory. Journal of Psychopathology and Behavioral Assessment, 27(1), 45-52.

Haas, T. C., \& Ferreira, S. M. (2015). Federated databases and actionable intelligence: using social network analysis to disrupt transnational wildlife trafficking criminal networks. Security Informatics, 4(2), 1-14.

Hansson, S. O. (2011). Decision Theory: An Overview. In M. Lovric (Ed.), International Encyclopedia of Statistical Science. Berlin, Heidelberg: Springer-Verlag.

Hardin, G. (1968). The Tragedy of the Commons. Science, 162(3859), 1243-1248.

Harvey, R. (2016). Risks and Fallacies Associated with Promoting a Legalised Trade in Ivory. Politikon, 43(2), 215-229.

Hay, D. (1975). Albion's Fatal Tree: Crime and Society in Eighteenth-Century England. London, United Kingdom: Allen Lane.

Holt, C. A., \& Laury, S. K. (2002). Risk aversion and incentive effects. American Economic Review, 92(5), 1644-1655.

Honeycutt, J., \& Orndorff, R. (2016, 7 November). How to Age Bucks. Retrieved from https://www.realtree.com/deer-hunting/how-to-age-bucks\#sequence

Humphreys, J., \& Smith, M. L. R. (2018). Militarised Responses to the Illegal Wildlife Trade. In T. Reitano, S. Jesperson, \& L. Bird Ruiz-Benitez de Lugo (Eds.), 
Militarised Responses to Transnational Organised Crime : The War on Crime (pp. 25-42). Cham: Springer International Publishing.

INTERPOL. (2015). INTERPOL Contribution to the United Nations General Assembly Special Session (UNGASS) on the World Drug Problem. Retrieved from Lyon, France:

https://www.unodc.org/documents/ungass2016/Contributions/IGO/INTERPOL_Input _to_UNGASS_19NOV2015.pdf

Jachmann, H., \& Billiouw, M. (1997). Elephant Poaching and Law Enforcement in the Central Luangwa Valley, Zambia. Journal of Applied Ecology, 34(1), 233-244.

Järvelä, S., Ekman, I., Kivikangas, J. M., \& Ravaja, N. (2014a). A practical guide to using digital games as an experiment stimulus. Transactions of the Digital Games Research Association, 1(2).

Järvelä, S., Ekman, I., Kivikangas, J. M., \& Ravaja, N. (2014b). A practical guide to using digital games as an experiment stimulus. Transactions of the Digital Games Research Association, 1(2), 85-115.

Just, D. R. (2014). Introduction to behavioral economics. Hoboken, NJ: John Wiley \& Sons.

Kahneman, D., \& Tversky, A. (1979). Prospect Theory: An Analysis of Decision under Risk. Econometrica, 47(2), 263-291.

Kalron, N., \& Crosta, A. (2013). Africa's White Gold of Jihad: al-Shabaab and Conflict Ivory [Press release]. Retrieved from http://elephantleague.org/project/africas-white-goldof-jihad-al-shabaab-and-conflict-ivory/

Keane, A., Jones, J. P., Edwards-Jones, G., \& Milner-Gulland, E. J. (2008). The sleeping policeman: understanding issues of enforcement and compliance in conservation. Animal conservation, 11(2), 75-82.

Kenney, M. (2007). The Architecture of Drug Trafficking: Network Forms of Organisation in the Colombian Cocaine Trade. Global Crime, 8(3), 233-259. 
Kingdon, J., Happold, D., Butynski, T., Hoffmann, M., Happold, M., \& Kalina, J. (Eds.). (2013). Mammals of Africa. Volume V: Carnivores, Pangolins, Equids and Rhinoceroses (Vol. 5). London, UK: Bloomsbury Publishing.

Kock, M. D., \& Atkinson, M. W. (1993). Report on dehorning of black (Diceros bicornis) and white (Ceratotherium simum) rhinoceroses in Zimbabwe. Retrieved from Harare, Zimbabwe: http://www.rhinoresourcecenter.com/pdf_files/129/1293781295.pdf

Kock, M. D., Morkel, P., Atkinson, M., \& Foggin, C. (1995). Chemical Immobilization of Free-Ranging White Rhinoceros (Ceratotherium simum simum) in Hwange and Matobo National Parks, Zimbabwe, Using Combinations of Etorphine (M99), Fentanyl, Xylazine, and Detomidine. Journal of Zoo and Wildlife Medicine, 26(2), 207-219.

Kozlov, M. D., \& Johansen, M. K. (2010). Real Behavior in Virtual Environments: Psychology Experiments in a Simple Virtual-Reality Paradigm Using Video Games. Cyberpsychology, Behavior, and Social Networking, 13(6), 711-714.

Kreps, D. M. (1990). A course in microeconomic theory. Princeton, NJ: Princeton University Press.

Kurpiers, L. A., Schulte-Herbruggen, B., Ejotre, I., \& Reeder, D. M. (2016). Bushmeat and Emerging Infectious Diseases: Lessons from Africa. In F. M. Angelici (Ed.), Problematic Wildlife: A Cross-Disciplinary Approach (pp. 507-551). Switzerland: Springer.

Langholz, J. A. (1996). Economics, Objectives, and Success of Private Nature Reserves in Sub-Saharan Africa and Latin America. Conservation Biology, 10(1), 271-280.

Langholz, J. A., \& Lassoie, J. P. (2001). Perils and Promise of Privately Owned Protected Areas: This article reviews the current state of knowledge regarding privately owned parks worldwide, emphasizing their current status, various types, and principal strengths and weaknesses. BioScience, 51(12), 1079-1085. 
Leader-Williams, N., \& Milner-Gulland, E. (1993). Policies for the enforcement of wildlife laws: the balance between detection and penalties in Luangwa Valley, Zambia. Conservation Biology, 7(3), 611-617.

Lee, T. E., \& Roberts, D. L. (2016). Devaluing rhino horns as a theoretical game. Ecological Modelling, 337, 73-78.

Lemieux, A. M., \& Clarke, R. V. (2009). The International Ban on Ivory Sales and its Effects on Elephant Poaching in Africa. The British Journal of Criminology, 49(4), 451-471.

Lewis, D. M., \& Alpert, P. (1997). Trophy Hunting and Wildlife Conservation in Zambia. Conservation Biology, 11(1), 59-68.

Lindsey, P. A., \& Taylor, A. (2011). A study on the dehorning of African rhinoceros as a tool to reduce the risk of poaching. Pretoria, South Africa: Republic of South Africa, Department of Environmental Affairs.

Linklater, W. L., \& Rudman, S. A. (2017). Experimental games to understand illegal hunting for wildlife commodities. Paper presented at the Human Dimensions of Wildlife, Estes, Colorado.

Linklater, W. L., Rudman, S. A., \& Jackson, C. (2017). Using experimental games to understand criminal hunting: Could rhino be saved by devaluing their horn? Unpublished manuscript.

Livingstone, E., Gomez, L., \& Bouhuys, J. (2018). A review of bear farming and bear trade in Lao People's Democratic Republic. Global Ecology and Conservation, 13, e00380.

Lunstrum, E. (2014). Green Militarization: Anti-Poaching Efforts and the Spatial Contours of Kruger National Park. Annals of the Association of American Geographers, 104(4), 816-832.

Manning, R. B. (1993). Hunters and poachers: A cultural and social history of unlawful hunting in England 1485-1640. Oxford, UK: Oxford University Press. 
Martin, E. B. (1994). Rhino poaching in Namibia from 1980 to 1990 and the illegal trade in the horn. Pachyderm, 18, 39-51.

Mason, C. F., Bulte, E. H., \& Horan, R. D. (2012). Banking on extinction: endangered species and speculation. Oxford Review of Economic Policy, 28(1), 180-192.

McCall, R., \& Baillie, L. (2017). Ethics, Privacy, and Trust in Serious Games. In R. Nakatsu, M. Rauterberg, \& P. Ciancarini (Eds.), Handbook of Digital Games and Entertainment Technologies (pp. 611-640). Singapore: Springer Singapore.

McCarthy, M. A., \& Possingham, H. P. (2007). Active Adaptive Management for Conservation. Conservation Biology, 21(4), 956-963.

Menickellia, J., \& Hastie, P. A. (2014). The Impact of Two Curricular Models on Motivation, Engagement and Achievement in Physical Education. International Journal of Physical Education, Fitness and Sports, 3(2), 33-42.

Mesterton-Gibbons, M., \& Milner-Gulland, E. J. (1998). On the strategic stability of monitoring: implications for cooperative wildlife programmes in Africa. Proceedings of the Royal Society of London. Series B: Biological Sciences, 265(1402), 1237-1244.

Milliken, T., Emslie, R. H., \& Talukdar, B. (2009). African and Asian Rhinoceroses - Status, Conservation and Trade. Retrieved from https://www.cites.org/sites/default/files/eng/cop/15/doc/E15-45-01A.pdf

Milliken, T., Shaw, J., Emslie, R., Taylor, R., \& Turton, C. (2012). The South AfricaVietnam rhino horn trade nexus. Traffic, 134-136.

Milner-Gulland, E. (1999). How many to dehorn? A model for decision-making by rhino managers. Animal conservation, 2(2), 137-147.

Milner-Gulland, E., Beddington, J., \& Leader-Williams, N. (1992). Dehorning African rhinos: a model of optimal frequency and profitability. Proceedings of the Royal Society of London B: Biological Sciences, 249(1324), 83-87. 
Milner-Gulland, E., \& Leader-Williams, N. (1992). A model of incentives for the illegal exploitation of black rhinos and elephants: poaching pays in Luangwa Valley, Zambia. Journal of Applied Ecology, 29(2), 388-401.

Milner-Gulland, E., Leader-Williams, N., \& Beddington, J. (1994). Is dehorning African rhinos worthwhile? Pachyderm, 18, 52-58.

Model [Def. 9]. (n.d.). In Collins English Dictionary Online. Retrieved from https://www.collinsdictionary.com/dictionary/english/model

Murcott, M. (2017). Transformative Environmental Constitutionalism's Response to the Setting Aside of South Africa's Moratorium on Rhino Horn Trade. Humanities, 6(4), $1-15$.

Myerson, R. B. (1991). Game Theory: Analysis of Conflict. Cambridge, MA: Harvard University Press.

Nijman, V. (2010). An overview of international wildlife trade from Southeast Asia. Biodiversity and Conservation, 19(4), 1101-1114.

Njerekai, C., \& Mabika, P. (2016). A Review of the Global Trophy Hunting Procedures and Processes with Illustrations from Zimbabwe. African Journal of Hospitality, Tourism and Leisure, 5(1), 1-15.

Paulsen, D. J., Platt, M. 1., Huettel, S. A., \& Brannon, E. M. (2011). Decision-Making Under Risk in Children, Adolescents, and Young Adults. Frontiers in Psychology, 2, 1-6.

Paulsen, D. J., Platt, M. L., Huettel, S. A., \& Brannon, E. M. (2012). From risk-seeking to risk-averse: the development of economic risk preference from childhood to adulthood. Frontiers in Psychology, 3, 1-6.

Phelps, J., Biggs, D., \& Webb, E. L. (2016). Tools and terms for understanding illegal wildlife trade. Frontiers in Ecology and the Environment, 14(9), 479-489. 
Pires, S. F., \& Moreto, W. D. (2011). Preventing wildlife crimes: Solutions that can overcome the 'Tragedy of the Commons'. European Journal on Criminal Policy and Research, 17(2), 101-123.

profile.idnz.co.nz. (2018a). Community profile | Wellington City | profile.id. Retrieved from: https://profile.idnz.co.nz/wellington\#

profile.idnz.co.nz. (2018b). Community profile| The Wellington Region| profile.id. Retrieved from: https://profile.idnz.co.nz/greater-wellington

R Core Team. (2017). R: A language and environment for statistical computing: $\mathrm{R}$ foundation fro Statistical Computing. Retrieved from https://www.R-project.org/

Redpath, S. M., Gutiérrez, R. J., Wood, K. A., \& Young, J. C. (2015). Conflicts in conservation: navigating towards solutions. Cambridge, UK: Cambridge University Press.

Redpath, S. M., Keane, A., Andrén, H., Baynham-Herd, Z., Bunnefeld, N., Duthie, A. B., .. . Travers, H. (2018). Games as Tools to Address Conservation Conflicts. Trends in ecology \& evolution, 33(6), 415-426.

Regan, H. M., Ben-Haim, Y., Langford, B., Wilson, W. G., Lundberg, P., Andelman, S. J., \& Burgman, M. A. (2005). Robust decision-making under severe uncertainty for conservation management. Ecological Applications, 15(4), 1471-1477.

Relyea, R., \& Hoverman, J. (2006). Assessing the ecology in ecotoxicology: a review and synthesis in freshwater systems. Ecology Letters, 9(10), 1157-1171.

Rhino Rescue Project. (n.d.-a). About the Project. Retrieved from http://rhinorescueproject.org/about-the-project/

Rhino Rescue Project. (n.d.-b). Infusion in action: Probe placement varies between horns. Retrieved from http://rhinorescueproject.org/infusion-in-action/ 
Rivalan, P., Delmas, V., Angulo, E., Bull, L. S., Hall, R. J., Courchamp, F., . . LeaderWilliams, N. (2007). Can bans stimulate wildlife trade? Nature, 447(7144), 529-530.

Robinson, S. (2008). Conceptual modelling for simulation Part I: definition and requirements. Journal of the Operational Research Society, 59(3), 278-290.

Rosen, G. E., \& Smith, K. F. (2010). Summarizing the Evidence on the International Trade in Illegal Wildlife. EcoHealth, 7(1), 24-32.

Rubino, E. C., Pienaar, E. F., \& Soto, J. R. (2018). Structuring Legal Trade in Rhino Horn to Incentivize the Participation of South African Private Landowners. Ecological Economics, 154, 306-316.

Sandbrook, C., Adams, W. M., \& Monteferri, B. (2015). Digital games and biodiversity conservation. Conservation Letters, 8(2), 118-124.

Schneider, J. L. (2008). Reducing the Illicit Trade in Endangered Wildlife. Journal of Contemporary Criminal Justice, 24(3), 274-295.

Shaw, M., \& Rademeyer, J. (2016). A Flawed War: Rethinking 'Green Militarisation'in the Kruger National Park. Politikon, 43(2), 173-192.

Sheehy-Skeffington, J., \& Rea, J. (2017). How Poverty Affects People's Decision-making Processes. York, UK: Joseph Rowntree Foundation.

Sollund, R. (2016). Wildlife Trafficking in a Globalized World: An Example of Motivations and Modus Operandi from a Norwegian Case Study. In F. M. Angelici (Ed.), Problematic Wildlife: A Cross-Disciplinary Approach (pp. 553-570). Switzerland: Springer.

Sullivan, B. L., Aycrigg, J. L., Barry, J. H., Bonney, R. E., Bruns, N., Cooper, C. B., . . Kelling, S. (2014). The eBird enterprise: An integrated approach to development and application of citizen science. Biological Conservation, 169, 31-40. 
Sumaila, U. R. (1999). A review of game-theoretic models of fishing. Marine Policy, 23(1), $1-10$.

Susi, T., Johannesson, M., \& Backlund, P. (2007a). Serious Games : An Overview (HS- IKI TR-07-001). Retrieved from http://urn.kb.se/resolve?urn=urn:nbn:se:his:diva-1279

Susi, T., Johannesson, M., \& Backlund, P. (2007b). Serious Games : An Overview ((ISSN)). Retrieved from Skövde: http://urn.kb.se/resolve?urn=urn:nbn:se:his:diva-1279

Talukdar, B. K., Emslie, R., Bist, S. S., Choudhury, A., Ellis, S., Bonal, B. S., . . Barua, M. (2008). Rhinoceros unicornis. The IUCN Red List of Threatened Species 2008, e.T19496A8928657.

Taylor, A., Brebner, K., Coetzee, R., Davies-Mostert, H., Lindsey, P., Shaw, J., \& 't SasRolfes, M. (2014). The viability of legalising trade in rhino horn in South Africa. Retrieved from Pretoria, South Africa: http://www.rhinoresourcecenter.com/pdf_files/139/1398153747.pdf

The New Zealand Ministry of Education. (2006). OECD thematic review of tertiary education. Retrieved from https://www.oecd.org/newzealand/36441052.pdf

Tietenberg, T. H. (2006). Environmental and natural resource economics (7 ed.). Boston, MA: Addison-Wesley.

Tor, A., Gazal-Ayal, O., \& Garcia, S. M. (2010). Fairness and the Willingness to Accept Plea Bargain Offers. Journal of Empirical Legal Studies, 7(1), 97-116.

Trump, K. (2017). Poaching and illegal wildlife trade. Retrieved from Germany: https://mobil.wwf.de/fileadmin/fm-wwf/PublikationenPDF/WWF_Poaching_and_illegal_Wildlife_Trade.pdf

Turner, H., \& Firth, D. (2012). Bradley-Terry Models in R: The BradleyTerry2 Package. Journal of Statistical Software, 48(9), 1-21. 
van Strien, N. J., Manullang, B., Sectionov, Isnan, W., Khan, M. K. M., Sumardja, E., .. . Bradley Martin, E. (2008). Dicerorhinus sumatrensis. The IUCN Red List of Threatened Species 2008, e.T6553A12787457.

van Strien, N. J., Steinmetz, R., Manullang, B., Sectionov, Han, K. H., Isnan, W., . . Ellis, S. (2008). Rhinoceros sondaicus. The IUCN Red List of Threatened Species 2008, e.T19495A8925965.

Vermillion, S. D., Malak, R. J., Smallman, R., Becker, B., Sferra, M., \& Fields, S. (2017). An investigation on using serious gaming to study human decision-making in engineering contexts. Design Science, 3, e15.

Vermillion, S. D., Malak, R. J., Smallman, R., \& Fields, S. (2014). Linking Normative and Descriptive Research with Serious Gaming. Procedia Computer Science, 28, 204-212.

Warchol, G. L. (2004). The Transnational Illegal Wildlife Trade. Criminal justice studies, 17(1), 57-73.

Washburn, D. A. (2003). The games psychologists play (and the data they provide). Behavior Research Methods, Instruments, \& Computers, 35(2), 185-193.

Wheeler, S. (1976). Trends and Problems in the Sociological Study of Crime. Social Problems, 23(5), 525-534.

Wik, M., Aragie Kebede, T., Bergland, O., \& Holden, S. T. (2004). On the measurement of risk aversion from experimental data. Applied Economics, 36(21), 2443-2451.

Wildlife ACT. (2018). Rhino dehorning in progress. Retrieved from https://wildlifeact.com/blog/rhino-dehorning-explained/

Williams, P. (2001). Transnational criminal networks. In J. Arquilla \& D. Ronfeldt (Eds.), Networks and netwars: the future of terror, crime, and militancy (pp. 61-97). Santa Monica, CA: Rand. 
Wittemyer, G., Northrup, J. M., Blanc, J., Douglas-Hamilton, I., Omondi, P., \& Burnham, K. P. (2014). Illegal killing for ivory drives global decline in African elephants. Proceedings of the National Academy of Sciences, 111(36), 13117-13121.

Woodroffe, R., Thirgood, S., \& Rabinowitz, A. (2005). People and Wildlife, Conflict or Coexistence? : Conflict or Coexistence? Cambridge, UNITED KINGDOM: Cambridge University Press.

Wright, R., Erdal Tekin, V. T., McClellan, C., Dickinson, T., \& Rosenfeld, R. (2014). Less cash, less crime: Evidence from the electronic benefit transfer program. Cambridge, MA: National Bureau of Economic Research.

Yesuf, M., \& Bluffstone, R. A. (2009). Poverty, Risk Aversion, and Path Dependence in Low-Income Countries: Experimental Evidence from Ethiopia. American Journal of Agricultural Economics, 91(4), 1022-1037.

Zuur, A., Ieno, E., Walker, N., Saveliev, A., \& Smith, G. (2009). Mixed effects models and extensions in ecology with R. New York, NY: Spring-Verlag. 



\title{
7 Appendices
}

\section{Appendix A}

\author{
VICTORIA UNIVERSITY OF WELLINGTON HUMAN ETHICS APPROVAL
}

MEMORANDUM (REFERENCE \#: 0000025115)

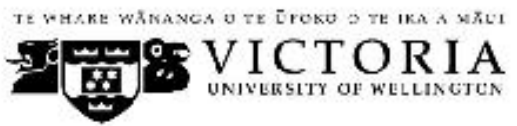

MEMORANDUM
Phone 044635480

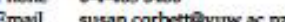

\begin{tabular}{l|l}
\hline TO & Sean Rudman \\
\hline COPY TO & $\begin{array}{l}\text { APProf Jack Robles } \\
\text { Dr Wayne Linklater }\end{array}$ \\
\hline FROM & AProf Susan Corbett, Convener, Human Ethics Committee \\
\hline DATE & 30 October 2017 \\
\hline PAGES & 1 \\
\hline SUBJECT & $\begin{array}{l}\text { Ethics Approval: 25115 } \\
\text { Experimental games to assess human behavioral responses to } \\
\text { economic devaluation strategies which alter supply and value of a } \\
\text { commodity such as rhino horn. }\end{array}$ \\
\hline
\end{tabular}

Thank you for your application for ethical approval, which has now been considered by the Standing Committee of the Human Ethics Committee.

Your application has been approved from the above date and this approval continues until 30 October 2020 . If your data collection is not completed by this date you should apply to the Human Ethics Committee for an extension to this approval.

Best wishes with the research.

Kind regards

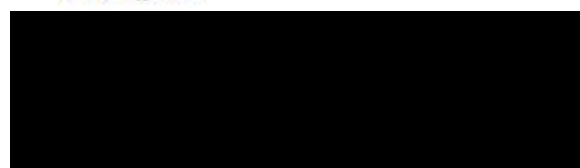

Susan Corbett

Convener, Victoria University Human Ethics Committee 
LUCKY-DIP PARTICIPANT INFORMATIOJN SHEET

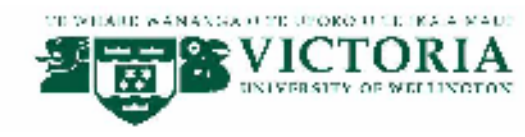
Experimental games to assess human behavioural responses to economic devaluation strategies

\section{INFORMATION FOR PARTICIPANTS OF 'LUCKY-DIP’ EXPERIMENTAI GAMES}

Thank you for your interest in this project. Please read this information before deciding whether or not to take part. If you decide to participate, thank you. If you decide not to talke part, thank you for considering my request.

Who am I?

My name is Sean Rudman and I am a Master's of Science student in Ecology \& Biodiversity at Victoria University of Wellington. This game is a research project.

What is the aim of the project?

The aim of this project is to determine how human harvesting behaviour changes in response to changes in supply and value of a desired harvestable commodity.

This research has been approved by the Victoria University of Wellington Human Ethics Committee [Reference \#: 0000025115].

How can you help?

If you agree to take part, you will participate in an experimental game of 'lucky-dip' format with other participants. A total of 10 participants will be presented with a series of 7 boxes each concealing different proportions of coloured balls. Blue balls will represent risk (their selection will result your removal from the game and loss of all acquired value). Yellow and red balls will represent differing monetary rewards of $\$ 2$ and $\$ 0.50$ respectively. The game will end once all participants have either selected a blue ball and been removed from the game, or decided to stop selecting balls. If you decide to stop playing before selecting a blue ball, you will receive the value of your accumulated balls.

Additionally, you may choose to enter a $\$ 250$ Prezzy Card prize draw.

The game will be video recorded for review later.

The total value of this game (sum of the value of all balls in all boxes) is [ $\$ 90$ or $\$ 140]$.

You can withdraw from the game at any time before the game begins, and any information you have provided will be retumed to you.

You can also withdraw while the game is in progress. However, it will not be possible to withdraw any information you have provided, or your contribution up to that point, as your behaviours may influence, or have influenced, that of other participants.

You will also be asked to complete a confidential post-game survey consisting of 6 questions that will require only 2 minutes of your time.

What will happen to the information you give? 


\section{Appendix B continued}

This research is confidential. This means that the researchers will be aware of your identity but the research data will be combined and your identity will not be revealed in any reports, presentations, or public documentation. However, you should be aware that in small projects your identity might be obvious to others in your community. By participating, you are disclosing that you are 18 years of age or older, and give consent for us to video participants playing the game and use the outcomes of these games and our observations of participant behaviour in this research. Your participation will remain completely confidential.

Only the primary investigator (myself) and the project supervisors will have access to the data and videos. The video recordings will only be used by the primary researchers to review the games for data, and will not be shown in any public space or conference.

Personal details will be collected only for those who wish to request a copy of the final report. All personal details will be received separately from the game and survey data. This ensures that your participation and answers to the survey questions are confidential.

The surveys, game summaries, consent forms, recordings and any personal details will be kept securely, and destroyed on $20^{\text {th }}$ November 2019.

Payments will be made in cash at the time of the game, and therefore we will not require personal payment details from participants.

What will the project produce?

The information from my research will be used in my Master's thesis and academic publications and conferences. Note that the video recordings will not be presented in any public space or conference.

If you accept this invitation, what are your rights as a research participant?

You do not have to accept this invitation if you don't want to. If you do decide to participate, you have the right to:

- an information brochure that fully explains the context of the research following the completion of your participation;

- ask for the video recorder to be turned off at any time during the game;

- withdraw from the game before it begins, with all your information returned;

- withdraw from the game while it is in progress. However, it will not be possible to withdraw the information or contribution you have provided up to that point;

- ask any questions about the study at any time;

- read any reports of this research by emailing the researcher to request a copy.

If you have any questions or problems, who can you contact?

If you have any questions, either now or in the future, please feel free to contact either:

Student:

Name: Sean Rudman

Role: Postgraduate Investigator

Email: rudmansean@myvrw.ac.nz

Human Ethics Committee information

If you have any concerns about the ethical conduct of the research you may contact the Victoria University HEC Convenor: Associate Professor Susan Corbett. Email susan.corbett@vuw.ac.nz or telephone +64-4-463 5480 .
Supervisor:

Name: Dr Wayne Linklater

Role: Associate Professor

Phone: +6444635233 ext. 8575

Email: Wayne.Linklater@vin.ac.nz 
LUCKY-DIP GAME CONSENT FORM FOR PARTICIPANTS

\author{
F国Q VICTORIA \\ Experimental games to assess human behavioural responses to \\ economic devaluation strategies

\section{CONSENT TO PARTICIPATE IN A 'LUCKY-DIP' EXPERIMINTAL GAME}

This consent form will be held until $20^{\text {th }}$ November 2019.

Researcher: Sean Rudman, School of Biological Sciences, Victoria University of Wellington.

- I have read the Information Sheet and the project has been explained to me. My questions have been answered to my satisfaction. I understand that I can ask further questions at any time.

- I agree to take part in a video recorded 'lucky-dip' game.

I understand that:

- I must be at least 18 years of age or older to participate.

- I may withdraw from this study at any point. However, if I withdraw after the game has begun my participation and information cannot be returned or retracted.

- The identifiable information I have provided will be destroyed on $20^{\text {th }}$ November 2019 .

- Any information I provide will be kept confidential to the researcher and the supervisor.

- I understand that the results will be used for a Masters thesis. Additionally, the results may be used in academic publications and presented to conferences (video recordings will not be publicly presented).

- My name will not be used in reports, nor will any information that would identify me.

- I would like to receive a copy of the final report, and have added my email Yes $\square$ No $\square$ address below.

- I would like to be entered in the $\$ 250$ Prezzy Card prize draw, and have Yes $\square$ No $\square$ added my email address below.

Signature of participant:

Name of participant:

Date:

Email 
Appendix D

POST-GAME INFORMATION BROCHURE FOR PARTICIPANTS THAT EXPLAINS THE CONTEXT OF THE RESEARCH

\section{The Games}

In the game you have just played:

You played the role of a poacher.

Karori Park represented a wildlife reserve and each stake represented a rhino.

The total value of each game was $\$ 250$. In this game, all stakes were worth the same value $(\$ 12.50)$. In other games, some stakes were worthless and other remainder are worth more.

Example: If 10 stakes are worthless, then the remaining 10 stakes are worth $\$ 25$ each so that the total value is still $\$ 250$.

In this way, each game represents a wildlife reserve with a different ratio of devaluation.

We are looking at how people's behaviour and motivation differs in response to different levels of devaluation.

Your contribution

Your decisions and behaviors of how to play the game, how many stakes were found and their fate, provides some insight in to the behavioral response of poachers to devaluation strategies.

\section{Who We Are}

About Us

While this project focuses on rhino conservation, the results could potentially be applied to other illegally killed and traded species such as elephant and pangolin. We hope to make a positive difference to the world of conservation.

We thank you greatly for your cooperation and participation. It is very much appreciated!

\section{Contact Us}

Sean Rudman (Postgraduate Investigator)

Email: sean.rudman@vuw.ac.nz

Dr. Wayne Linklater (Associate Professor) Phone: 044635150

Email: wayne.linklater@vuw.ac.nz

\begin{tabular}{|c|c|}
\hline 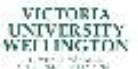 & $\begin{array}{l}\text { VICTORIA UNIVERSITY } \\
\text { OF WELLINGTON }\end{array}$ \\
\hline 要要8 & $\begin{array}{l}\text { Kelburn } \\
\text { Wellington, } 6012\end{array}$ \\
\hline
\end{tabular}

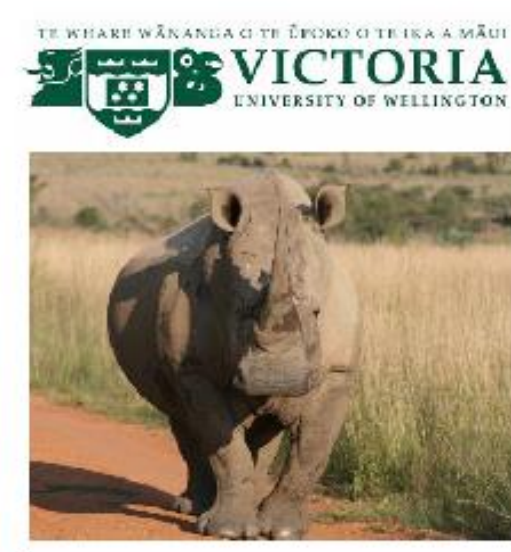

WILDLIFE

COMMODITY

DEVALUATION

STRATEGIES TO

PREVENT RHINO

POACHING

Post-game Information Brochure 


\section{Appendix D continued}

\section{Rhino poaching: a conservation issue}

Rhino are killed across Africa and Asia for their horn which is primarily trafficked to, and sold in, South-East Asia.

Rhino horn is falsely used medicinally to cure illnesses such as cancer, fever, impotence and hangovers.

It is also used ornamentally for products such as cultural knife handles in Yemen.

Rhino horn sells for up to USD $\$ 60,000$ per kilogram!

In 2015, 1,215 rhino were killed in South Africa alone!

Security has had some success at protecting rhino in reserves. However, this alone has not been sufficient.

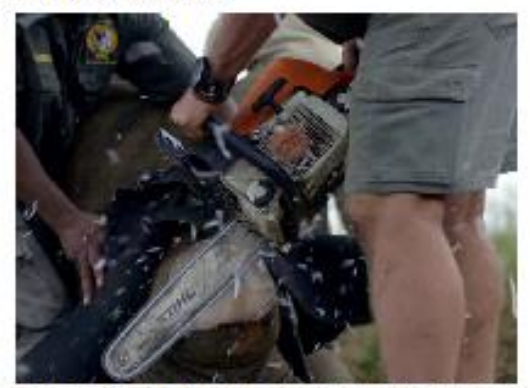

Image 1. The humane horn removal process Wildlife managers remove a horn with a chainsaw from a tranquilized rhino.
Wildlife commodity devaluation strategies are proposed as a solution to this crisis!

These strategies involve modifying the horn so that it is worthless to poachers.

Devaluation is achieved through either:

1. humane horn removals (Image 1) so there is no horn for poachers to harvest.

2. infusing horns with toxins (Image 2) and a dye so that the horn is not suitable for human consumption or ornamental use.

These strategies are used to reduce the reward to poachers and therefore discourage illegal hunting.

\section{However, there is a possible} alternative outcome.

When only some horns can be devalued, the supply of valuable horns is reduced.

Economics tells us that when a commodity becomes rarer it also becomes more valuable.

Therefore, devaluation strategies could increase the market value of untreated horn and encourage further hunting, resulting in a worse outcome.

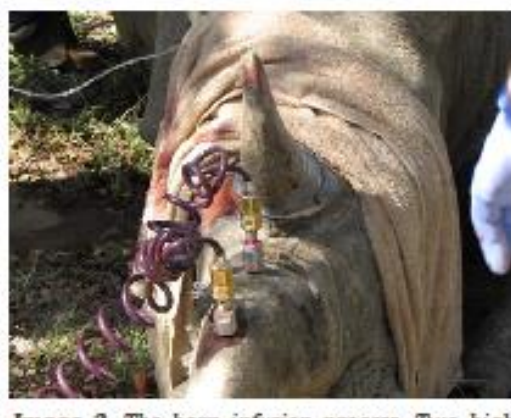

Image 2. The horn infusion process. Two hig pressure infusion probes inserted into the horn infuse a toxin into the horn.

We aim to assess two key questions:

1. When do devaluation strategies work?

2. What is the optimal mixed protection strategy including both devaluation and security?

This cannot be assessed with actual poachers due to their secretive nature and the danger involved in doing so.

Therefore, we are using experimental games and economic modelling.

Experimental games allow us to alter the supply, reward and risk of a harvesting activity to replicate a devaluation strategy, and investigate peoples' behaviour. 


\section{Appendix E}

\section{LUCKY-DIP POST-GAME PARTICIPANT SURVEY}

Player Number:

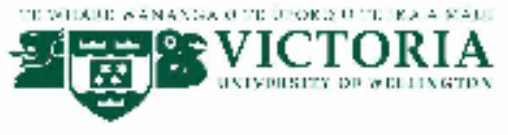

\section{'Luckv-dip' post-game Survev}

1. Reflect on your experience and how you played the game.

a. What would you have done differently?

b. Did you cooperate or work with anyone during the game? If so, what was your cooperation deal?

2. If you selected a red ball, did you keep it?

If so, why?

3. If you selected a red ball, did you return it to its box?

If so, why?

4. Why did you decide to 'harvest' from the box(es) that you did? 


\title{
SCAVENGER-HUNT GAME INFORMATION SHEET FOR PARTICIPANTING ORGANISATIONS
}

\author{
S4 OVICTORIA \\ E $\cdots$ : \\ Experimental games to assess human behavioural responses to \\ economic devaluation strategies

\section{INFORMATION FOR ORGANISATION PROVIDING PARTICIPANTS FOR 'SCAVENGER-HUNT' EXPERIMENTAL GAMES}

Thank you for your interest in this project. Please read this information before deciding whether or not to take part. If you decide to participate, thank you. If you decide not to take part, thank you for considering my request.

Who am I?

My name is Sean Rudman and I am a Master's of Science student in Ecology \& Biodiversity at Victoria University of Wellington. This game is a research project.

What is the aim of the project?

The aim of this project is to determine how human harvesting behaviour changes in response to changes in supply and value of a desired harvestable commodity.

This research has been approved by the Victoria University of Wellington Human Ethics Committee [Reference \#: 0000025115].

How can you help?

If you agree to take part, we will recruit members of your organisation as participants for an experimental game of a 'scavenger-hunt' format. Members of your organisation will be invited to participate and only those that consent will participate.

One organisation will provide twenty consenting searcher participants. They will have to search [a WCC Park] in pairs for hidden items across three half-hour long time periods separated by two half-hour breaks. The entire activity will take 2.5 hours. A proportion of the hidden items will have a monetary value (up to $\$ 125$ each), while the remainder of the items will be worthless. The value of the items found by all searchers will be donated to their organisation. To claim the value of an item it must be delivered to a designated buyer (a game coordinator).

A second organisation will provide two protectors who will aim to protect the hidden items and catch searchers. If a searcher is caught by a protector they will be removed from the game and lose the value of any collected but unclaimed items. At the end of the game, the value of the items not found by searchers will be donated to the organisation of the two protectors. 


\section{Appendix F continued}

The total possible value of this game (sum of the value of all hidden items) is $\$ 250$.

You, as an organisation, can withdraw from the game at any time before the game begins, and any information you have provided will be retumed to you.

You, as an organisation, can also withdraw while the game is in progress. However, it will not be possible to withdraw any information you have provided, or your contribution up to that point.

Withdrawal from the game will require informing either the supervisor or primary investigator (student) of this via email or phone call. The contact details for these individuals are included on this form.

What will happen to the information you give?

This research is confidential. This means that the researchers will be aware of the identity of your organisation, but your organisation will not be revealed in any reports, presentations, or public documentation. By participating, you are giving consent for us to use the outcomes of these games and our observations of participant behaviour in this research. Your organisations participation will remain completely confidential.

Only participants who are 18 years of age or older will be able to participate.

Only the primary investigator and the project supervisors will have access to the data.

You may elect to receive a copy of the final report for this project, and will have to provide an email address in order to receive this.

Payments to your organisation will be made via bank transfer to an account of your choosing.

The game summaries, consent forms and your organisation's details will be kept securely, and destroyed on $20^{\text {th }}$ November 2019.

What will the project produce?

The information from my research will be used in my Master's thesis and academic publications and conferences.

If you accept this invitation, what are your rights as a research participant?

You do not have to accept this invitation if you don't want to. If you do decide to participate, you have the right to:

- an information brochure or presentation from myself that fully explains the context of the research following the completion of your participation;

- withdraw from the game before it begins, with all your information returned;

- withdraw from the game while it is in progress. However, it will not be possible to withdraw the information or contribution you have provided up to that point;

- ask any questions about the study at any time;

- read any reports of this research by emailing the researcher to request a copy. 


\section{Appendix F continued}

If you have any questions or problems, who can you contact?

If you have any questions, either now or in the future, please feel free to contact either:

Student:

Name: Sean Rudman

Role: Postgraduate Investigator

Email: rudmansean@myvuw.ac.nz
Supervisor:

Name: Dr Wayne Linklater

Role: Associate Professor

School: Biological Sciences

Phone: +64 44635233 ext. 8575

Email: Wayne.Linklater@vuw.ac.nz

Human Ethics Committee information

If you have any concems about the ethical conduct of the research you may contact the Victoria University HEC Convenor: Associate Professor Susan Corbett. Email susan.corbett@vuw.ac.nz or telephone +64-4-4635480. 


\title{
Appendix G
}

\section{SCAVENGER-HUNT GAME CONSENT FORM FOR PARTICIPATING ORGANISATIONS}

\author{
(and

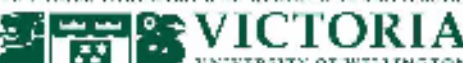

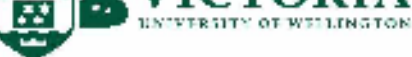

\section{Experimental games to assess human behavioural responses to economic devaluation strategies}

\section{CONSENT FROM ORGANISATION INVOLVED IN EXPERIMENTAL GAME}

This consent form will be held until $20^{\text {th }}$ November 2019 .

Researcher. Sean Rudman, School of Biological Sciences, Victoria University of Wellington.

- I have read the Information Sheet and the project has been explained. My questions have been answered to my satisfaction. I understand that I can ask further questions at any time.

- I agree for this organisation to be involved in this project, and for members of this organisation to be recruited as participants for a 'scavenger-hunt' experimental game.

I understand that:

- Members of this organisation will be recruited as participants for an experimental game.

- This organisation may withdraw from this study at any point. However, if this organisation withdraws after the game has begun, the contribution and information it has provided cannot be returned or retracted.

- The identifiable information provided by the organisation and participants will be destroyed on $20^{\text {th }}$ November 2019 .

- Any information provided will be kept confidential to the researcher and the supervisor.

- I understand that the results will be used for a Masters thesis. Additionally, the results may be used in academic publications and presented to conferences.

- The organisations name will not be used in reports, nor will any information that would identify the organisation.

- I would like to receive a copy of the final report, and have added an email Yes $\square$ No $\square$ address below.

Signature of organisation representative:

Name of organisation representative:

Name of organisation:

Date:

Email 


\title{
Appendix H
}

\section{SCAVENGER-HUNT GAME INFORMATION SHEET FOR PARTICIPANTS}

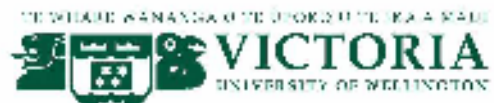 \\ Experimental games to assess human behavioural responses to \\ economic devaluation strategies

\section{INFORMATION FOR PARTICIPANTS OF 'SCAVENGER-HUNT' EXPERIMENTAL GAMES}

Thank you for your interest in this project. Please read this information before deciding whether or not to take part. If you decide to participate, thank you. If you decide not to take part, thank you for considering my request.

Who am I?

My name is Sean Rudman and I am a Master's of Science student in Ecology \& Biodiversity at Victoria University of Wellington. This game is a research project.

What is the aim of the project?

The aim of this project is to determine how human harvesting behaviour changes in response to changes in supply and value of a desired harvestable commodity.

This research has been approved by the Victoria University of Wellington Human Ethics Committee [Reference \#: 0000025115].

How can you help?

If you agree to take part, you will participate as either a searcher or a protector in an experimental game of a 'scavenger-hunt' format with other participants. In pairs, searchers must search [a WCC Park] alongside 9 other searcher pairs for hidden items across three halfhour long time periods separated by two half-hour breaks. The entire activity will take 2.5 hours. A proportion of the hidden items will have a monetary value (up to $\$ 125$ each), while the remainder of the items will be worthless. The value of the items found by all searchers will be donated to their organisation. To claim the value of an item it must be delivered to a designated buyer participant (a game coordinator).

Two protectors recruited from another organisation will work as a pair with the aim of protecting the hidden items and catching searchers. If a searcher is caught by a protector they will lose the value of any collected but unclaimed items. At the end of the game, the value of the items not found by searchers will be donated to the organisation of the two protectors.

The total possible value of this game (sum of the value of all hidden items) is $\$ 250$.

Additionally, you may choose to personally enter a \$250 Prezzy Card prize draw. 


\section{Appendix H continued}

You can withdraw any time before the game begins, and any information you have provided will be returned to you.

You can also withdraw while the game is in progress. However, it will not be possible to withdraw any information you have provided, or your contribution up to that point, as your behaviours may influence, or have influenced, that of other participants.

You will also be asked to complete a confidential post-game survey consisting of 6 questions that will only require 2 minutes of your time.

What will happen to the information you give?

This research is confidential. This means that the researchers will be aware of your identity but the research data will be combined and your identity will not be revealed in any reports, presentations, or public documentation. However, you should be aware that in small projects your identity might be obvious to others in your community. By participating, you are agreeing that you are 16 years of age or older, and give consent for us use the outcomes of these games and our observations of participant behaviour in this research. Your participation will remain completely confidential.

Only the primary investigator (myself) and the project supervisors will have access to the data.

Personal details will be collected only for those who wish to request a copy of the final report. All personal details will be received separately from the game and survey data. This ensures that your participation and answers to the survey questions are confidential.

Payments to organisations will be made via bank transfer to an account of their choosing.

The surveys, game summaries, consent forms and any personal details will be kept securely, and destroyed on $20^{\text {th }}$ November 2019.

What will the project produce?

The information from my research will be used in my Master's thesis and academic publications and conferences.

If you accept this invitation, what are your rights as a research participant?

You do not have to accept this invitation if you don't want to. If you do decide to participate, you, as an organisation, have the right to:

- an information brochure or presentation by myself that fully explains the context of the research following the completion of your participation;

- withdraw from the game before it begins, with all your information returned;

- withdraw from the game while it is in progress. However, it will not be possible to withdraw the information or contribution you have provided up to that point;

- ask any questions about the study at any time;

- read any reports of this research by emailing the researcher to request a copy. 


\section{Appendix $H$ continued}

If you have any questions or problems, who can you contact?

If you have any questions, either now or in the future, please feel free to contact either:

Student:

Name: Sean Rudman

Role: Postgraduate Investigator

Email: rudmansean@myvuw.ac.nz
Supervisor:

Name: Dr Wayne Linklater

Role: Associate Professor

School: Biological Sciences

Phone: +6444635233 ext. 8575

Email: Wayne.Linklater@vuw.ac.nz

Human Ethics Committee information

If you have any concerns about the ethical conduct of the research you may contact the Victoria University HEC Convenor: Associate Professor Susan Corbett. Email susan.corbett@vuw.ac.nz or telephone +64-4-4635480. 


\title{
SCAVENGER-HUNT GAME CONSENT FORM FOR PARTICIPATING ORGANISATIONS
}

\author{
FOICTORIA

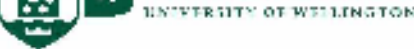

\section{Experimental games to assess human behavioural responses to economic devaluation strategies}

\section{CONSENT TO PARTICIPATE IN A 'SCAVENGER-HUNT' EXPERIMENTAL GAME}

This consent form will be held until $20^{\text {th }}$ November 2019 .

Researcher: Sean Rudman, School of Biological Sciences, Victoria University of Wellington.

- I have read the Information Sheet and the project has been explained to me. My questions have been answered to my satisfaction. I understand that I can ask further questions at any time.

- I agree to take part in a 'scavenger-hunt' experimental game.

I understand that:

- I must be at least 16 years of age or older to participate.

- I may withdraw from this study at any point. However, if I withdraw after the game has begun my participation and information cannot be returned or retracted.

- The identifiable information I have provided will be destroyed on $20^{\text {th }}$ November 2019 .

- Any information I provide will be kept confidential to the researcher and the supervisor.

- I understand that the results will be used for a Masters thesis. Additionally, the results may be used in academic publications and presented to conferences.

- My name will not be used in reports, nor will any information that would identify me.

- I would like to receive a copy of the final report, and have added my email Yes $\square$ No address below.

- I would like to be entered in the $\$ 250$ Prezzy Card prize draw, and have Yes $\square$ No $\square$ added my email address below.

Signature of participant:

Name of participant:

Date:

Email: 
Appendix J

SCAVENGER-HUNT POST-GAME PARTICIPANT SURVEY

Team colour:

Date:

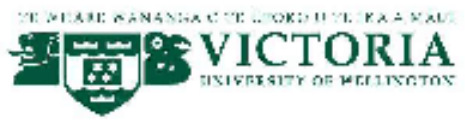

'Scavenger Hunt' post-game Survev

1. Motivation: In this game, 10 stakes were worth $\$ 25$ each, and the reaming 10 stakes were worth nothing.

How motivated were you to find and claim the value of stakes in this game? (circle)

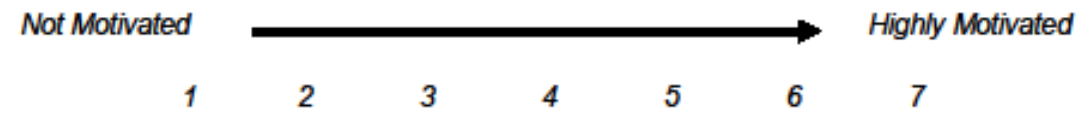

How motivated would you have been to find and claim the value of stakes if all 20 stakes had been worth an equal $\$ 12.50$ each? (circle)

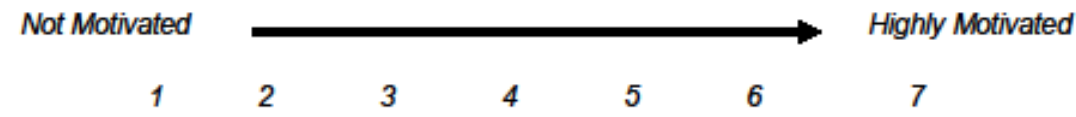

How motivated would you have been to find and claim the value of stakes if only 2 stakes had been worth $\$ 125$ each, with the remaining 18 worth nothing? (circle)

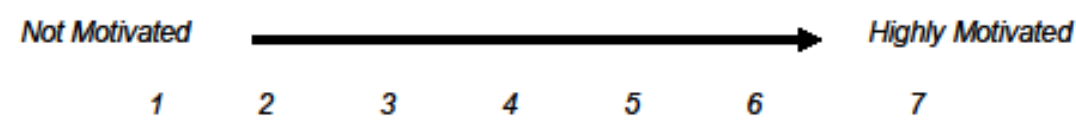

2. Explain the answers you have provided above 


\section{Appendix $\mathbf{J}$ continued}

3. Reflect on your experience and how you played the game.

a. How would you have played the game differently?

b. Did you cooperate or work with any other teams? If so, how did you cooperate or work with other teams?

4. If you found a worthless stake, did you leave it alone?

Yes: If so, why?

No: If so, why?

5. If you found a worthless stake, did you lay it down?

Yes: If so, why?

No: If so, why? 


\section{Appendix K}

\section{ONLINE SURVEY FOR RECREATIONAL HUNTERS}

$10 / 19 / 2018$

Qualtrices Survey Sottware

Consent

\section{Experimental games to assess human behavioural responses to economic devaluation strategies}

\section{INFORMATION FOR PARTICIPANTS OF ANONYMOUS HUNTING SURVEY}

You are invited to take part in this research. Please read this information before deciding whether or not to take part. If you decide to participate, thank you. If you decide not to participate, thank you for considering this request.

Who am I?

My name is Sean Rudman and I am a Master's of Science student in Ecology \& Biodiversity at Victoria University of Wellington. This research project is work towards my thesis.

What is the aim of the project?

The aim of this project is to determine how hunter behaviour changes in response to changes in supply and value of a desired harvestable commodity. The survey is designed to study how hunter behaviour responds to changes in the risk of failure and the value of the reward.

This research has been approved by the Victoria University of Wellington Human Ethics Committee [Reference \#: 0000025115].

How can you help?

If you agree to take part, you will complete an anonymous online survey. The survey will ask you questions about hunting. You have been invited to participate because you are a part of New Zealand's hunting fraternity who routinely consider the risks and rewards of hunting. You are relevant to our research, and most likely to benefit from our findings. The survey will take you 10-12 minutes to complete.

Additionally, you may choose to enter a \$250 Prezzy Card prize draw.

What will happen to the information you give?

This research is anonymous. This means that nobody, including the researchers will be aware of your identity. By answering it, you are agreeing that you are 16 years of age or older and you are giving consent for us to use your responses in this research. Your answers will remain completely anonymous 
and unidentifiable. Once you submit the survey, it will be impossible to retract your answer. Please do not include any personal identifiable information in your responses.

Personal details will be collected only for those who wish to enter the $\$ 250$ Prezzy Card prize draw and/or request a copy of the final report. These details will be received separately from the survey data. This ensures that your answers to the survey questions remain anonymous.

What will the project produce?

The information from this survey will be used in my Master's thesis, academic publications and conferences.

Only the primary investigator (myself) and the project supervisors will have access to the data. The survey responses will be kept securely, and destroyed on 20th November 2019.

If you have any questions or problems, please feel free to contact either?

Student:

Sean Rudman

Postgraduate Investigator

sean.rudman@vuw.ac.nz

Supervisor:

Dr. Wayne Linklater

Associate Professor

wayne.linklater@vuw.ac.nz

$+6444635150$

Human Ethics Committee information

If you have any concerns about the ethical conduct of the research you may contact the Victoria

University HEC Convenor: Dr Judith Loveridge. Email hec@vuw.ac.nz or telephone +64-4-463 6028.

Yes I Consent

O No I do not Consent

\section{Scenario 1}

\section{$\underline{\text { Scenario }}$}

(This scenario is fictional)

You have been offered free entry in to a balloted hunting event on Stewart Island. You will be hunting white-tailed deer (Odocoileus virginiamus borealis) stags for venison. Venison is a highly valuable commodity with annual exports valued at $\$ 165$ million. 
When you are drawn from the ballot, you will be randomly assigned two or the four hunting blocks. You can pick which of the two blocks you want to hunt in. Older deer are usually larger with more venison therefore they are worth more.

Within your chosen block, you are permitted to hunt one animal of your choice that you can locate. A buyer will be present to purchase your kill from you. The animals can be placed into the 5 following age categories. These are outlined below, along with the value the buyer is willing to provide per age class.

- Yearling $(\$ 100)$

- 2-years old $(\$ 200)$

- 3-years old $(\$ 300)$

- 4-years old $(\$ 500)$

- $5+$ years old $(\$ 1,100)$

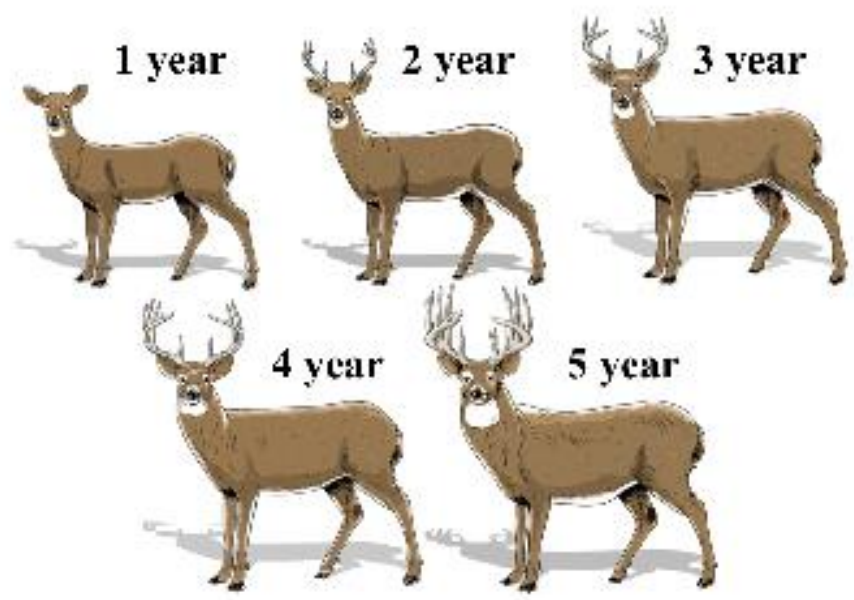

The four blocks all contain 20 white-tailed stags, and the total value of venison in each block equals $\$ 4,000$.

- Block 1: All 20 stags are 2-years old.

- Block 2: Ten of the 20 stags (50\%) are yearlings. The other 10 stags $(50 \%)$ are 3-years old.

- Block 3: Fifteen of the 20 stags (75\%) are yearlings. The other 5 stags $(25 \%)$ are 4-years old.

- Block 4: Eighteen of the 20 stags $(90 \%)$ are yearlings. The other 2 stags $(10 \%)$ are at least 5 -years old.

Block 1

You may chose to hunt in either Block 1 or Block 2. Both blocks contain a total of $\$ 4,000$ worth of venison. Which block do you choose? 


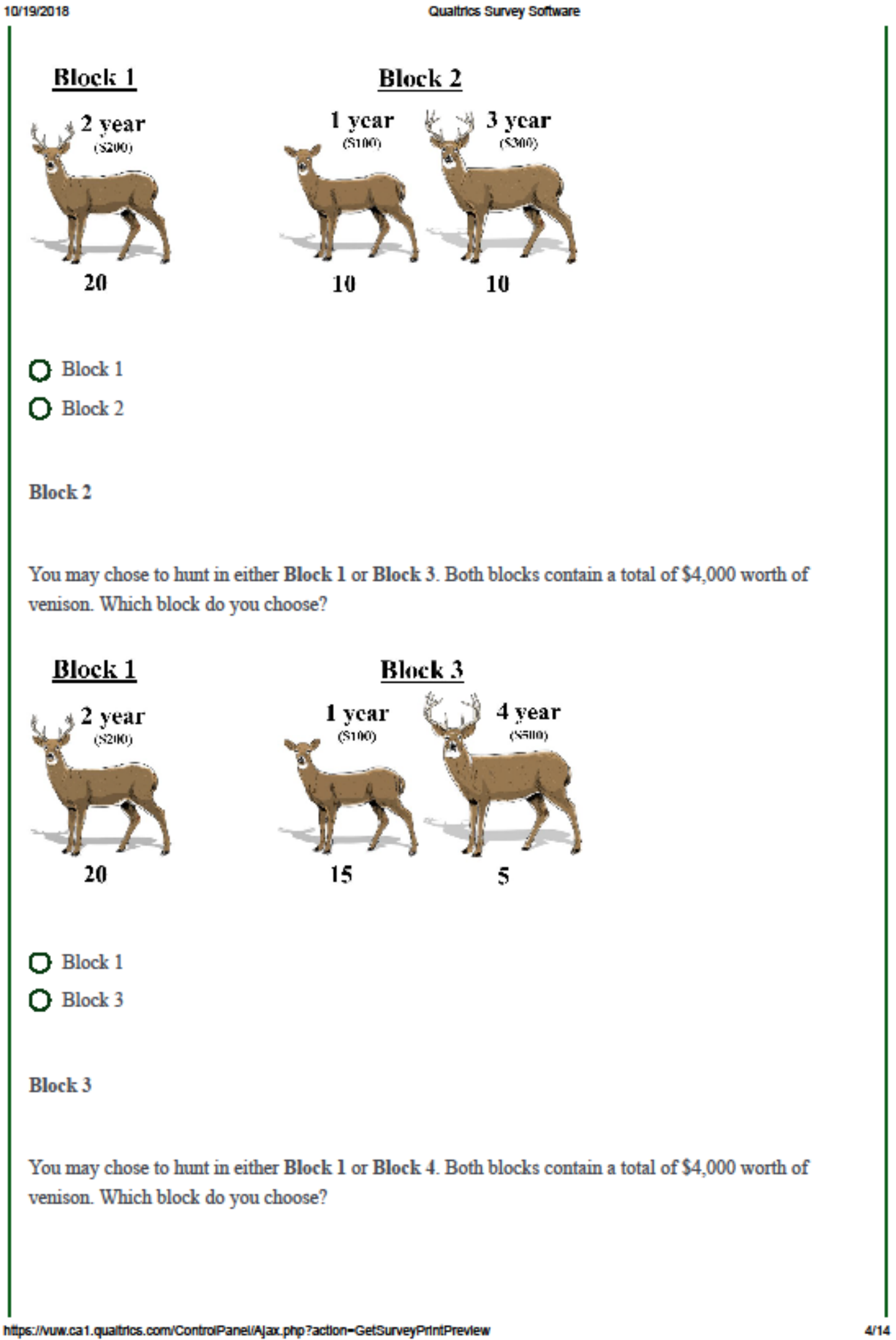




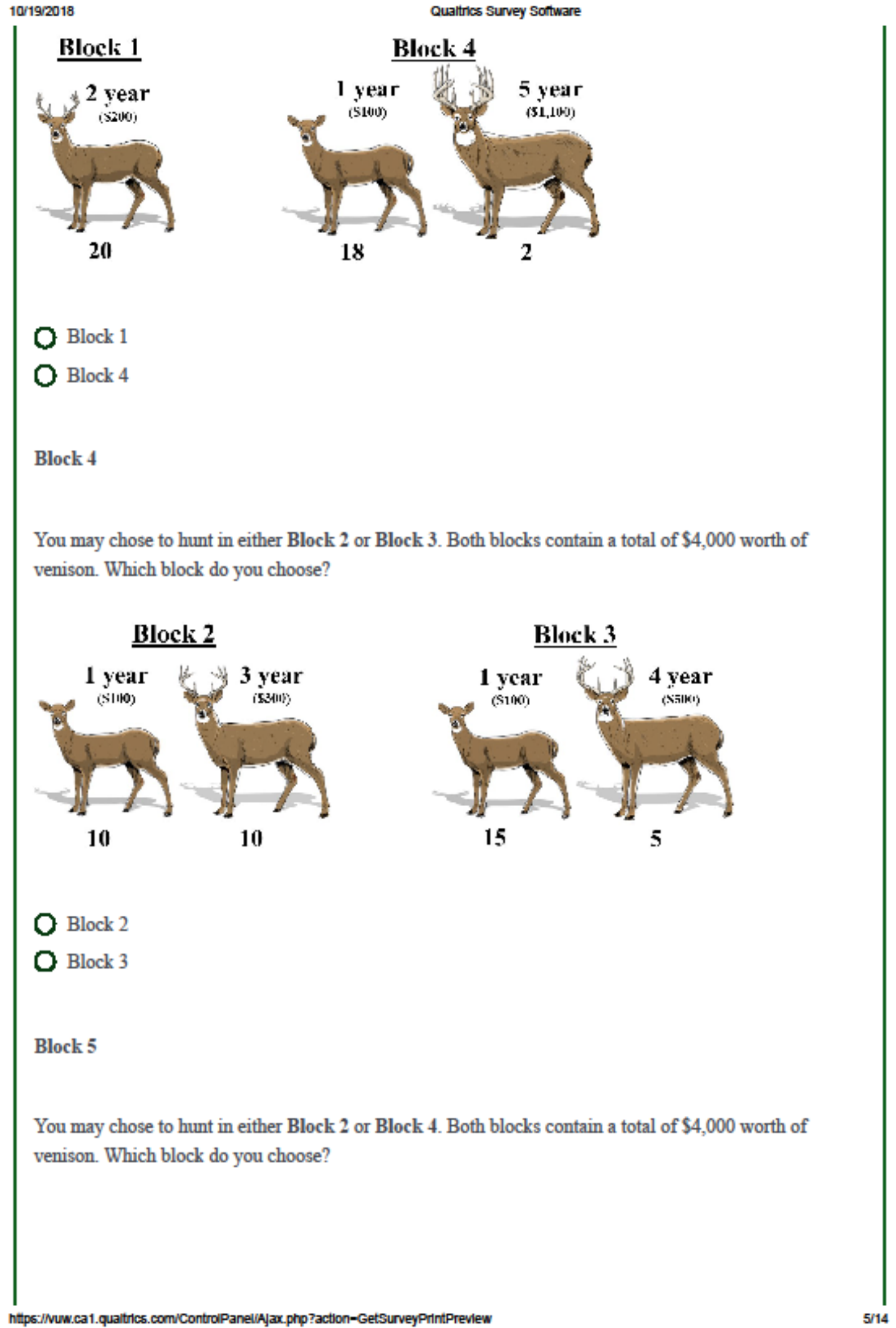




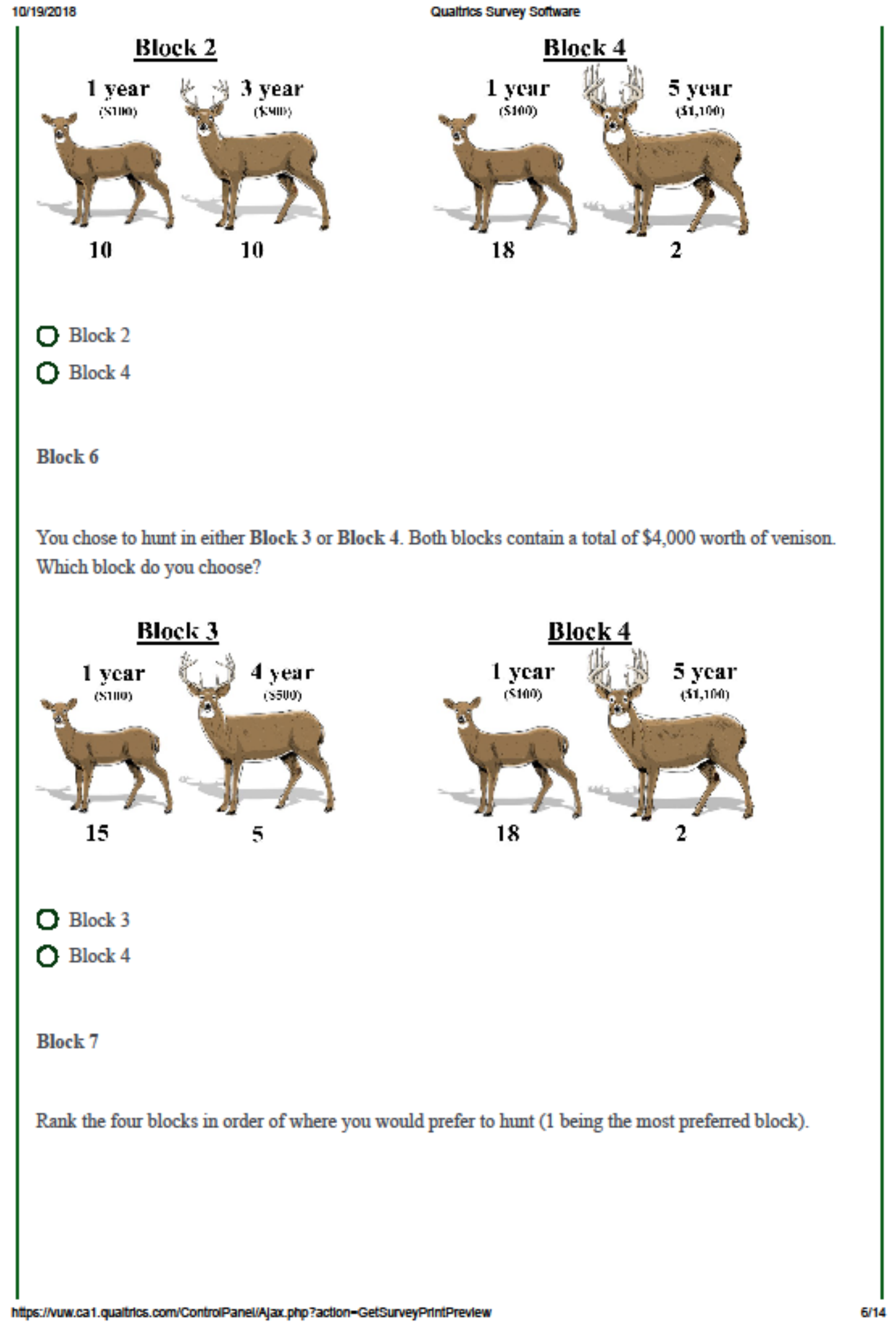




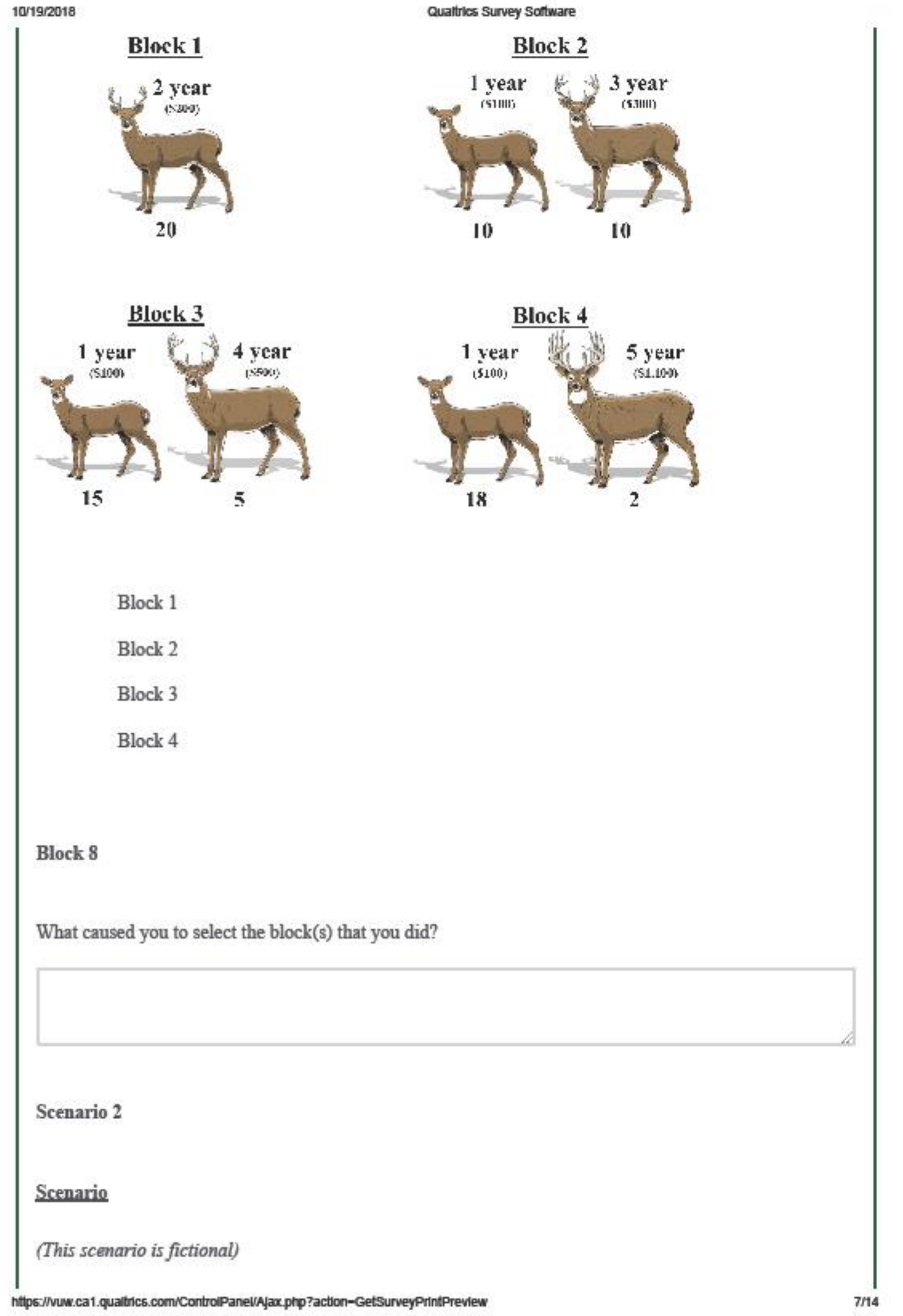


You are taking part in a hunting ballot for a trophy hunt of white-tailed deer (Odocoileus virginianus borealis) on Stewart Island. This will be the first time you are hunting white-tailed deer. There are four hunting blocks that each permit 5 white-tailed deer stags to be hunted. Each stag is numbered, and you will be blindly selecting a stag to hunt from a bowl that contains each stags' number. Each block has a different proportion of mature stags and yearlings. The rules of the ballot are:

The hunt will take place over 3 days. Each hunter will be permitted to hunt for one stag on one of the 3 days. The ballot occurs in two stages:

1. Hunting Ballot: The first ballot is to be allowed to hunt that day - there are 20 hunters but only 16 hunting spaces $(80 \%)$.

2. Stag Ballot: If you receive a hunting space, the second ballot is to select which of the 5 stags you can hunt.

The yearlings effectively have no trophy. If you select a yearling from the stag ballot, you have two choices:

1. Return the number to the bowl - you receive a continuation and can immediately re-select a stag to hunt on day 2 . However, there is a chance you will select the same animal again.

2. Discard the number from the bowl - that animal cannot be selected again, but you must re-enter the hunting ballot. You will again only have an $80 \%$ chance of receiving a hunting slot on day 2 before you can re-select a stag from the stag ballot

\section{Block 9a}

You have received a hunting slot on day 1 . Of the 5 available stags, 4 are yearlings ( $80 \%)$ and 1 is a mature stag $(20 \%)$.

Click the random number generator below. A number between 1-4 means you have selected a yearling. The number 5 means you have found the mature stag.

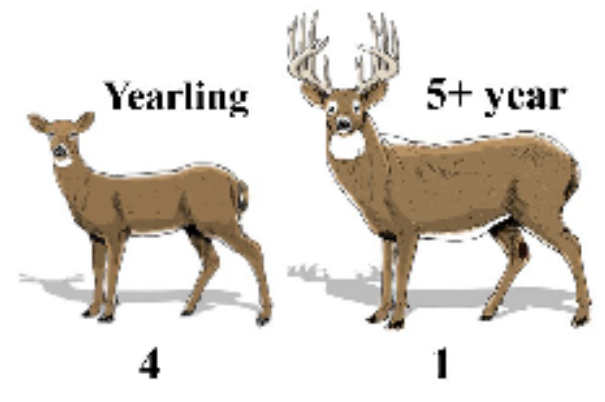


Block 9b

You randomly selected animal 2. You have selected a YEARLING. You may either:

Return the number to the bowl - you can immediately re-select a stag to hunt on day 2 from the stag ballot. But, you might select the same stag again

Discard the number from the bowl - remove that stag from the bowl, but there is a $20 \%$ chance that you will not be able to select a stag to hunt on day 2 .

What do you do?

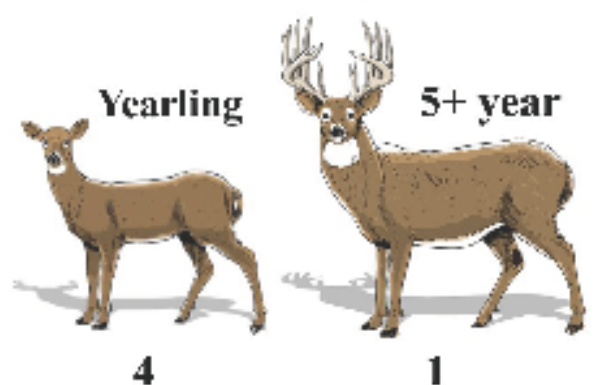

Return the number to the bowl

Discard the number from the bowl

Block 9c

Why did you decide to \$ $\{q: / / Q I D 12 / C h o i c e G r o u p / S e l e c t e d C h o i c e s\} ?$

Block 10a

You have received a hunting slot on day 1 . Of the 5 available stags, 3 are yearlings (60\%) and 2 are mature stags (40\%). 


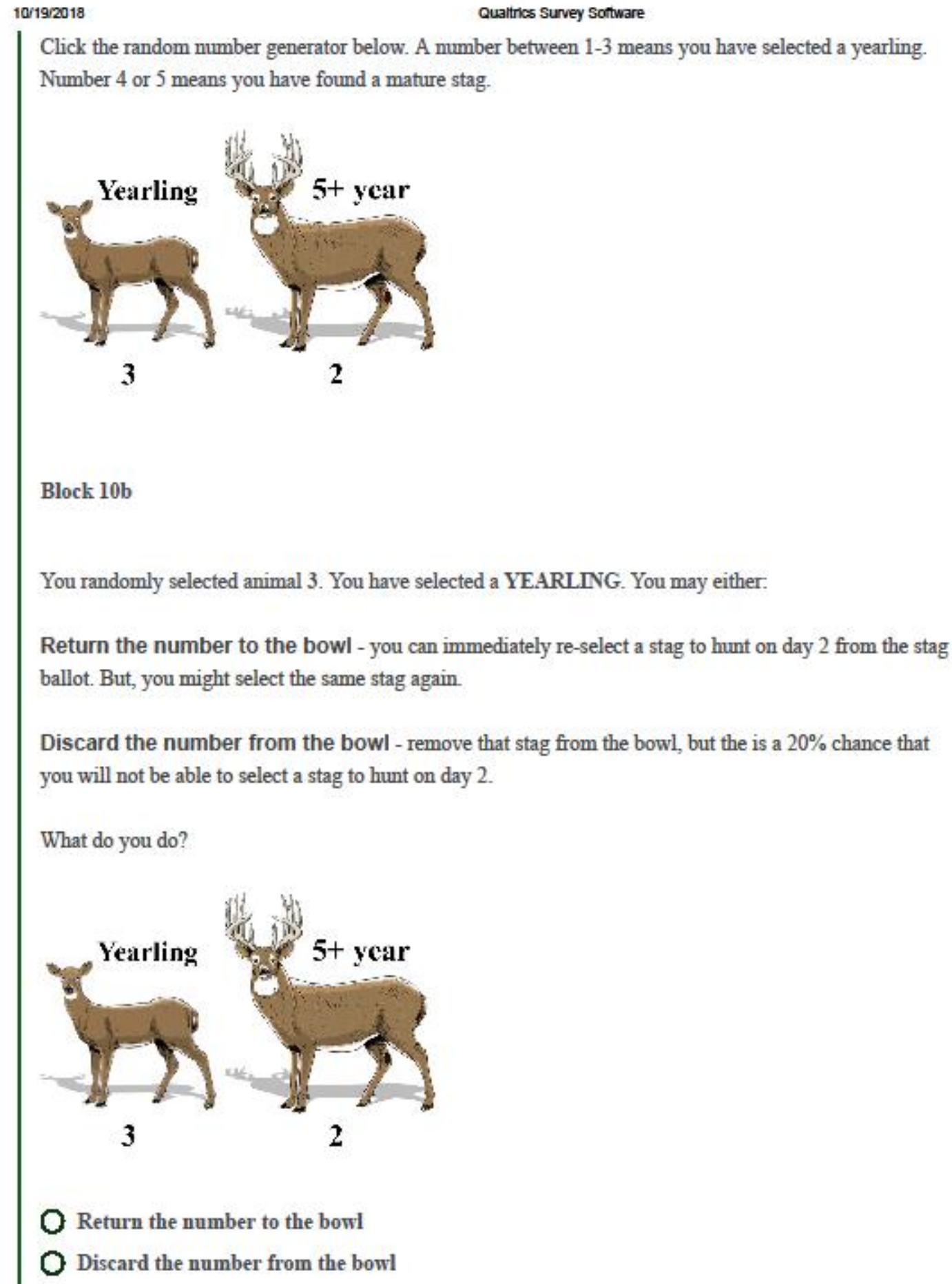

Block 10c 
Why did you decide to \$\{q://ID15/ChoiceGroup/SelectedChoices $\}$ ?

Block lla

You have received a hunting slot on day 1 . Of the 20 available stags, 2 are yearlings ( $40 \%$ ) and 3 are mature stags $(60 \%)$.

Click the random number generator below. Number 1 or 2 means you have selected a yearling. A number between 3-5 means you have found a mature stag.

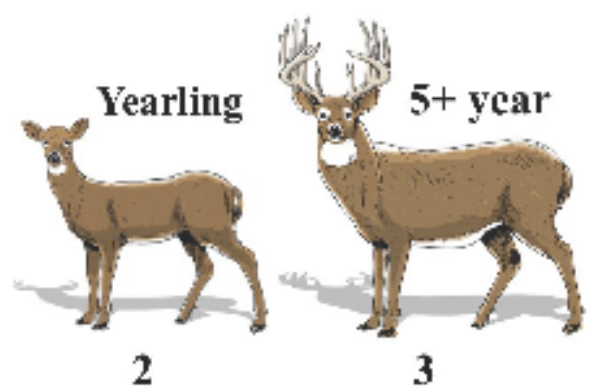

\section{Block llb}

You randomly selected animal 1. You have selected a YEARLING. You may either:

Return the number to the bowl - you can immediately re-select a stag to hunt on day 2 from the stag ballot. But, you might select the same stag again.

Discard the number from the bowl - remove that stag from the bowl, but there is a $20 \%$ chance that you will not be able to select a stag to hunt on day 2 .

What do you do? 


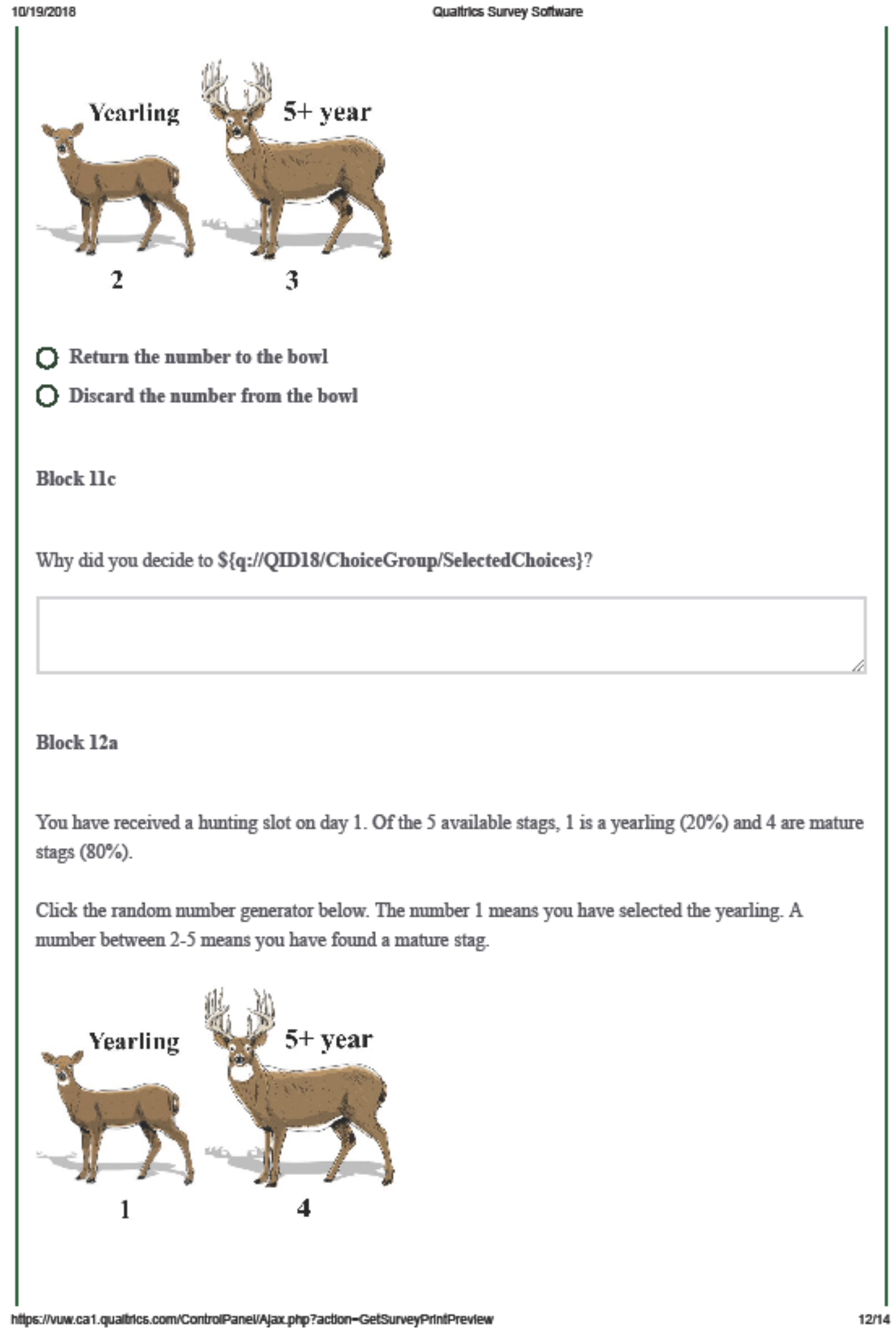




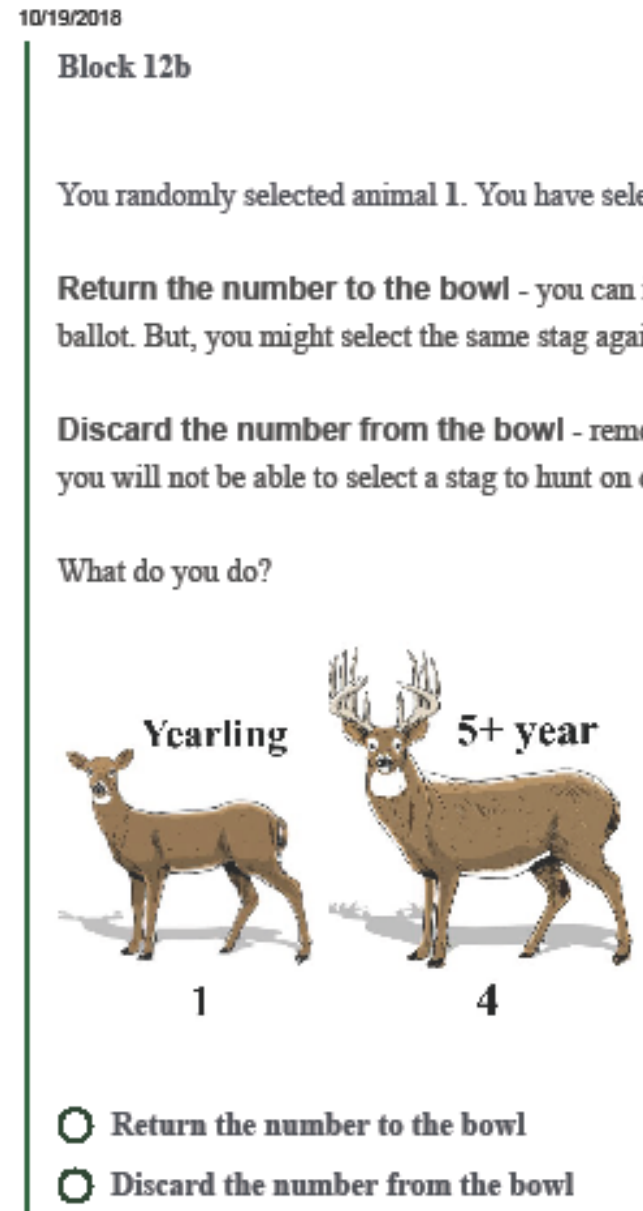

Block 12

You randomly selected animal 1 . You have selected a YEARLING. You may either:

Return the number to the bowl - you can immediately re-select a stag to hunt on day 2 from the stag ballot. But, you might select the same stag again

Discard the number from the bowl - remove that stag from the bowl, but there is a $20 \%$ chance that you will not be able to select a stag to hunt on day 2 .

What do you do?

Discard the number from the bowl

Block 12c

Why did you decide to \$\{q://QID39/ChoiceGroup/SelectedChoices\}?

Context

The survey is complete. Would you like to read more about the context of this research and the reasons behind it?

O Yes

No

htlps:/Vuw.ca1.qualtics.com/ControlPanel/Ajax php?action-GetSurveyPrintPreview 


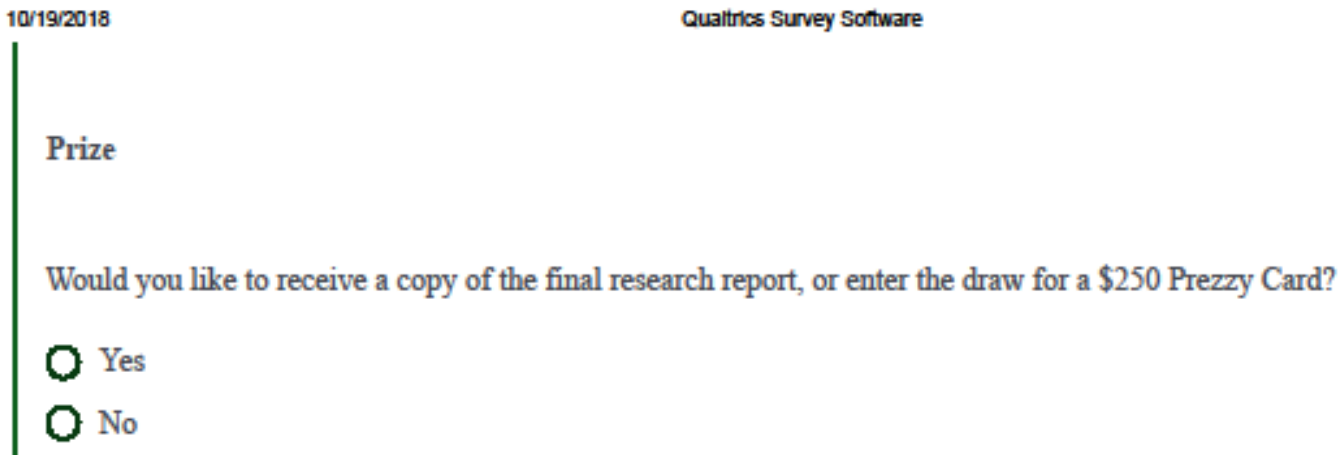

Powered by Qualtrics 